\title{
Prediction of wave-induced loads on ships: Progress and challenges
}

\author{
P. Temarel \\ Fluid Structure Interactions Group, University of Southampton, UK \\ W. Bai \\ Department of Civil and Environmental Engineering, National University of Singapore, \\ Singapore \\ A. Bruns \\ Danish Maritime Authority, Copenhagen, Denmark \\ Q. Derbanne \\ Bureau Veritas, France \\ D. Dessi \\ CNR-INSEAN Marine Technology Research Center, National Research Council, \\ Rome, Italy \\ S. Dhavalikar \\ Indian Register of Shipping, Mumbai, India \\ N. Fonseca \\ Marintek, Norway \\ T. Fukasawa \\ Osaka Prefecture University, Japan \\ X. Gu \\ China Ship Scientific Research Centre, China \\ A. Nestegard \\ Marine Structures, DNV GL, Norway \\ A. Papanikolaou \\ Ship Design Laboratory, National Technical University of Athens, Greece \\ J. Parunov \\ Faculty of Mechanical Engineering \& Naval Architecture, University of Zagreb, Croatia \\ K.H. Song \\ Ship \& Plant Technology Center, Korean Register, Korea \\ S. Wang \\ ABS, Houston, USA
}

\begin{abstract}
The aim of this paper is to critically assess the methods used for the evaluation of wave-induced loads on ships examining analytical, numerical and experimental approaches. The paper focuses on conventional ocean going vessels and loads originating from steady state and transient excitations, namely slamming, sloshing and green water, for the latter, and including extreme or rogue waves, as well as the more occasional loads following damage. The advantages and disadvantages of the relatively simpler potential flow approaches against the more time consuming CFD methods are discussed with reference to accuracy, modelling nonlinear effects, ease of modelling and of coupling with structural assessment procedures, suitability for long term response prediction and suitability for integration within design and operational decision making. The paper also assesses the uncertainties involved in predicting wave-induced loads and the probabilistic approaches used for the evaluation of long term response and fatigue analysis. The current design practice is reviewed and the role of numerical prediction methods within the classification framework and goal based design approach discussed. Finally the suitability of current developments in prediction methods to meet the needs of the industry and future challenges is assessed.
\end{abstract}




\section{Keywords}

Wave loads, hydroelasticity, slamming, sloshing, design, uncertainty

\section{Introduction}

The structural integrity of vessels operating at sea, more often than not in extreme weather, is of paramount importance in order to ensure the safety of passengers and crew, the timely and safe delivery of cargo and avoid adverse environmental consequences due to catastrophic or nearly catastrophic failures. The designer has to ensure that global structural strength, as well as local structural details, are capable of withstanding operational and environmental loads over the life time of the ship, whilst balancing these requirements against economic and environmental emission driven pressures calling for lighter and more efficient ship structures and structural arrangements. The largely deterministic approach to global structural strength, involving static equilibrium of the ship on a trochoidal wave crest and trough, is no longer fit for purpose. The structural design of ships to detailed classification requirements is gradually displaced by risk or goal based approaches, leading to designs of quantifiable safety level verified by validated numerical methods. The drivers for such change include drastic changes in ship type, size and complexity, increased knowledge and more data on the environment, more data on model and real ship behavior in waves, a wide range of numerical models capable of simulating the physics of ship behavior, even under extreme conditions, and ever increasing computational power enhancing the suitability of these methods in design applications. The fundamental factor fuelling these developments is the ability to acquire and generate more data, and the trends are pointing to increasingly more data.

This state of affairs informed the discussions of the ISSC Committee I.2: Loads in the past three years or so (ISSC, 2015a). The Committee comprises academics and practitioners, providing an ideal background to ponder on a range of issues. These are, for example, the accuracy of predictions from nonlinear potential flow methods compared to RANS methods and, the so called, hybrid methods driven by balancing accuracy against numerical efficiency. Other issues are, the versatility of available numerical methods to successfully, or not as the case may be, simulate the phenomena associated with seakeeping and wave loads and the relative weaknesses with reference to realistic environmental modelling (ISSC, 2015b). Furthermore, the gradual move towards simulating the complete performance of the vessel in waves, rather than just individual aspects such as rigid body response, slamming, sloshing, green water etc are issues of interest. In addition, the necessity of simulating loads due to extreme events, the suitability of numerical methods to provide accurate input to long term predictions and the usefulness of data acquired from measurements and numerical predictions, with its associated uncertainties, are issues that dominated the discussions of the committee. We firmly believe that these are issues of great importance to researchers and designers alike, from the viewpoint of providing guidance to the researcher on where to focus future efforts and to the designer on navigating through a plethora of methods and data.

In this paper we will endeavor to deal with these issues by reviewing progress made in the past 3 years in the subject area of wave-induced loads. The structure of this paper follows along traditional lines to direct readers to their area of interest. Nevertheless, it should be noted that (i) the versatility of numerical methods to model more than one phenomenon, e.g. sloshing and slamming, and (ii) the focus of investigations in dealing with as complete fluid-structure interaction modelling as possible, results in some overlaps between the sections of this paper. The generic computational methods are discussed in section 2 and 3, with the latter focusing on hdroelasticity. The transient loads relating to slamming, green water and sloshing, and, where applicable their coupling to the rigid body response, are reviewed in sections 4,5 and 6 . The loads in abnormal or freak waves are examined in section 7. Section 8 is focused on experimental and full-scale measurements, whilst the particular loads due to damage following collision or grounding are in Scetion 9. The life-time related issues, namely probabilistic methods, design waves and fatigue loads are discussed in Sections 10 and 11, including weather routing. Finally section 12 investgigates uncertainties in loads and loading conditions, with conclusive remarks in Section 13. 


\section{Computational methods for evaluating wave-induced loads}

Thanks to dramatic advances of computer science and technology during recent years, the numerical wave tank has attracted great interest from researchers. Computational Fluid Dynamics (CFD) making use of the Reynolds Averaged Navier Stokes (RANS) equations, although computationally intensive, features significantly in many investigations. Nonlinear potential flow solutions continue to be developed and used. Most of the numerical investigations deal with twodimensional (2D) problems due to constraints from computational resources and/or CPU time consumption associated with three-dimensional (3D) modelling. This is particularly so when dealing with fully nonlinear modelling and irregular waves.

The problem of wave-induced loads on ships with forward speed is one of the most demanding in ship hydrodynamics, especially when considering the excitation by moderate or large amplitude waves. In theoretical/numerical approaches, this requires the proper consideration of the forward speed effects on ship motions and loads and of a variety of nonlinearities related to the large amplitude ship motions and to ship's actual wetted surface, as well to the change of the free surface of the incoming waves and their interaction with the moving ship. Due to the complications of the above set problem, simplifications and engineering solutions are often adopted. This implies that the exact nonlinear seakeeping and large amplitude wave-induced loads problem may be still considered unsolved, except for special cases. A brief review of related theoretical and numerical methods is outlined in this section.

Greco \& Lugni (2012) developed a 3D seakeeping numerical solver to handle occurrence and effects of water-on-deck and bottom slamming. It couples (A) the rigid ship motions with (B) the water flowing along the deck and (C) bottom slamming events. Problem A is studied with a 3D weakly nonlinear potential flow solver based on the weak-scatterer hypothesis. Problem B, and so local and global induced green water loads, are investigated by assuming shallow water conditions onto the deck. Problem $\mathrm{C}$ is examined through a Wagner type wedge impact analysis. For coupling between A and B, the external seakeeping problem furnishes the initial and boundary conditions to the in-deck solver in terms of water level and velocity along the deck profile; in return, the shallow water problem makes available to the seakeeping solver the green water loads to be introduced as additional loads into the rigid motion equations. For the coupling between $\mathrm{A}$ and $\mathrm{C}$, the instantaneous ship configuration and its kinematic and dynamic conditions with respect to the incident waves fix the parameters for the local impact problem; in return, the slamming and water entry pressures are integrated on the vessel region of interest and introduced as additional loads into the rigid motion equations. The developed solver has been applied to the problems of a dam breaking inside a closed tank and to the wave-ship interaction problem with/without water-on-deck occurrence for validation. Obtained results are compared with experimental data. Subsequently, Greco et al. (2012) carried out experimental and numerical investigations on a patrol boat at rest or travelling in head regular and irregular waves. In these studies motion RAOs, relative motions and occurrence, features and loads of water-ondeck, bottom slamming and flare slamming events, as well as added resistance in waves, were investigated. The analysis is systematic covering a range of Froude numbers, wave length $(\lambda)$ to ship length $\left(L_{P P}\right)$ ratios and wave steepness values. The main parameters that affect the global and local quantities are identified and possible issues in terms of, for example, water-on-deck severity and structural consequences are determined. Different slamming behaviors were identified, depending on the spatial location of the impact on the vessel, namely single-peak, churchroof and double-peak behaviors. A bottom slamming criterion is assessed. Predicted and measured bottom pressures at section 18 (section 20 corresponding to FP) are shown in Fig.1 when the vessel is travelling at Froude number 0.189, for three different wave lengths and wave steepness 0.15 . The major discrepancies with the experiments are discussed, and the importance of viscous hull damping and flare impact for the most violent conditions is emphasized. Inclusion of these effects has improved the numerical solution.

Hanninen et al. (2012) studied an interface capturing VOF solution for a passenger ship advancing in steep $\left(\mathrm{kA}=0.24, \mathrm{k}\right.$ : wave number, $\mathrm{A}$ : wave amplitude) and short waves $\left(\lambda / \mathrm{L}_{\mathrm{PP}}=\right.$ 0.16 ), with the focus on estimating quantitative uncertainties for the longitudinal distributions of the first-third harmonic wave loads in the bow region. The computations were performed with the commercial flow solver ISIS-CFD. The uncertainty distributions obtained reveal that even the uncertainty of the first harmonic wave load varies significantly along the bow region. It is 
shown that the largest local uncertainties of the first harmonic wave load relate to the differences in the local details of the propagating and deforming encountered waves along the hull. The authors also discussed the challenges that were encountered in the quantification of the uncertainties for this complex flow case. Hanninen et al. (2014) considered the capability of their interface capturing method to predict local ship wave loads in short and steep waves $(\mathrm{kA}=0.24$, $\left.\lambda / \mathrm{L}_{\mathrm{PP}}=0.16\right)$ by comparing with experimental results. The computations were performed with an unstructured RANS solver that models free surface flows with a VOF method. It was shown qualitatively that the solution behavior of the computed results is reasonable, even though the results can depend on the location of the computational points within the surface area of a pressure sensor. The agreement between computed and measured results is good at all the 10 locations on the bow of the ship. The characteristics of the wave loads vary between the sensor locations. Impact-type as well as smoother behavior of the loading is captured well by the numerical method. The work by Kim et al. (2012a), on estimating the long term midships bending moments and Oberhagemann et al (2012) on embedding time domain field methods in extreme value predictions are discussed in Section 10.2.

Greco et al. (2013) developed a 3D domain decomposition strategy to deal with violent waveship interactions involving water-on-deck and slamming occurrence. This couples a linear potential seakeeping solver, in the outer domain, and Lagrangian markers for the body motion, in the inner domain where slamming, water-on-deck and free surface fragmentation may occur, involving important flow nonlinearities. The field solver combines an approximated projection method with a level set technique for the free surface evolution. Main features of the weak and strong coupling algorithms are described with special focus on the boundary conditions for the inner solver. Two ways of estimating the nonlinear loads by the N-S method are investigated, on the basis of an extrapolation technique and an interpolation marching cubes algorithm, respectively. The domain decomposition is applied for the case of a patrol boat travelling in head regular waves and compared against water-on-deck experiments in terms of flow evolution, body motions and pressure on the hull. The solver was successfully verified by comparison against the linear potential flow solution in the case of incident waves with small steepness and validated against model tests in the case of steeper waves.

Rajendran et al. $(2013,2014)$ presented a time domain numerical method based on strip theory. They calculated the probability distributions of relative motions and bending moments of a cruise ship in a set of extreme seas. Their approach includes two levels of complexity. The simpler one combines linear radiation and diffraction forces with nonlinear Froude-Krylov forces, hydrostatic forces and shipping of green water at the bow. Cummins' Impulse Response Function (IRF) formulation is used to represent the radiation forces. The second approach is a generalization of the first and the effects of body nonlinearity are considered by a simplified method, namely the memory functions, infinite frequency added masses and the radiation restoring coefficients are evaluated at each time instant as functions of the instantaneous wetted surface. A similar procedure is used to calculate the diffraction forces. In their latter model, the first order Froude Krylov pressures are replaced with a second order model. The 2nd order Froude-Krylov pressures are integrated up to the exact wetted surface area for each time instant. The nonlinear radiation and diffraction effects on the responses are analyzed by comparing the fully nonlinear results with the numerical predictions assuming linear radiation and diffraction forces. The short term nonlinear responses are represented by empirical probability distributions, obtained from time domain simulations, and the quality of the predictions is assessed by comparing with model test data.

Seng \& Juncher Jensen (2013) developed a new approach, which requires reduced computational effort for the estimation of the short term statistical properties of the hull girder responses, as predicted by a free surface CFD solver. The approach, known as the MCF (model corrector factor), is an efficient alternative to the polynomial based response surface approach to the structural reliability analysis. The concept is to apply a predictor (e.g. a strip theory) to determine the most probable response conditioned wave sequence and the associated statistical properties. Thereafter, the predictor is applied to improve the evaluation of the responses for only a few selected wave sequences. An algorithm is proposed to support the selection process. To illustrate the process of transferring statistical properties to the CFD results (i.e. the corrector), an application to a 9400 TEU post Panamax container vessel is given using a nonlinear time do- 
main strip theory as the predictor. The discussion focuses on the usage and the implicit requirements of the MCF approach, especially when slamming induced responses are considered.

Finally, a series of research investigations referring to the seakeeping problem are also reviewed. Though without direct reference to loads, these methods contain important methodology developments or applications, and will allow the calculation of wave loads with some additional development work. Sun \& Faltinsen (2012) studied the steady and unsteady hydrodynamic problems of a semi-displacement ship with round bilge at high forward speed with a numerical method based on $2 \mathrm{D}+\mathrm{t}$ theory. The ship was forced to oscillate in heave in the unsteady problem. No incident waves were present. The non-viscous flow separation from the round bilge of the ship hull was simulated. The pressure on the hull surface was evaluated and the sectional hydrodynamic vertical forces were obtained. Good agreement was achieved between the present calculations and the experiments, although some discrepancies near the bow and stern were observed. The interaction between the local steady flow and unsteady flow are automatically included and the nonlinearities in both steady and unsteady flow were considered. Shao \& Faltinsen (2012) presented an alternative formulation of the boundary value problem for linear seakeeping and added resistance analysis based on a body-fixed coordinate system which does not involve the, so called, $\mathrm{m}_{\mathrm{j}}$ terms in the traditional formulation when an inertial coordinate system is applied. Numerical studies were carried out for a modified Wigley I hull, a Series 60 ship and the S175 container ship for moderate forward speeds where it is thought appropriate to use the double body flow as basis flow. Results for the forced heave and pitch oscillations, motion responses, and added resistance in head waves show good agreement with experiments and other numerical studies.

He \& Kashiwagi (2014) developed a time domain HOBEM for the 3D forward speed radiation and diffraction problems. Extensive results, including the exciting forces, added mass and damping coefficients, wave profiles and wave patterns for blunt and slender Wigley hulls with forward speed, are presented to validate the efficiency of the proposed 3D time domain approach. Model tests of the radiation and diffraction problems in a towing tank were also carried out. Computed numerical results show good agreement with the corresponding experimental data and other numerical solutions.

Liu et al. (2014a) presented a 3D nonlinear time domain method for the prediction of ship responses in waves based on the IRF concept. In this method, the wave excitation is decomposed into Froude-Krylov, radiation and diffraction forces. Incident wave forces are calculated through direct integration of the corresponding pressures over the instantaneous wetted surface. The radiation forces are obtained using the added mass and damping coefficients calculated by a 3D frequency domain code and transformed into the time domain by application of the IRF concept. Diffraction forces are obtained in a similar manner. Solving the six coupled nonlinear integro-differential equations of motion by a time integration method, motions in the six degrees of freedom (DoF) of the ship are obtained in the time domain. The validation of this method was conducted through applications to the S-175, DTC and KVLCC2 hulls. Good agreement was observed between the results of the present method, other numerical codes and available experimental data, which confirm the capability of the developed numerical approach to deliver reliable predictions.

Guo et al. (2012a) predicted the added resistance and ship motion of the KVLCC2 hull in head waves using the ISIS-CFD flow solver. The numerical results are analyzed in terms of added resistance, ship motions and wake flow. Both free to heave and pitch and fixed models are studied to investigate the contribution to added resistance from ship motions at different wavelengths, and the results show that ship motion-induced added resistance is negligible when the wavelength $\lambda<0.6 \mathrm{~L}_{\mathrm{PP}}$. Comparison with calculations based on strip theory and experimental results shows that RANS predicts the added resistance better in all wavelengths.

Simonsen et al. (2013) investigated the KCS container ship in calm water and regular head waves by means of experimental and CFD studies. The experimental study was conducted in FORCE Technology's towing tank in Denmark, and the CFD study was conducted using the URANS codes CFDSHIP-IOWA and Star-CCM+ as well as the potential theory code AEGIR. The wave conditions were chosen in order to study response in waves under resonance and maximum exciting conditions for three forward speeds. In the experiment, the heave and pitch motions and the resistance were measured together with wave elevation of the incoming wave. The results show that the ship responds strongly when the resonance and maximum exciting 
conditions are met. With respect to experimental uncertainty, the level for calm water is comparable to PMM uncertainties for manoeuvering testing while the level is higher in waves. Concerning the CFD results, the computation shows a very complex and time-varying flow pattern. Comparison with experimental data shows that the computed motions and resistance in calm water are in fair agreement with the measurement. In waves, the motions are still in fair agreement with measured data, but larger differences are observed for the resistance.

It has been expected that the correct forward speed hydrodynamic coefficients of a surface ship advancing in waves would be obtained if the 3D frequency domain forward-speed free surface Green's function (Brard, 1948) and the 3D forward speed Green's integral equation presented by Hong (2000) were jointly used. Hong et al. (2013b, 2014) showed that the line integral in the 3D forward speed Green's integral equation can properly be discretized using 8-node inner collocation $2^{\text {nd }}$ order BEM. Their numerical results were obtained using Brard's Green function expressed through complex exponential integrals (Guevel et al., 1979). Predictions of the heave damping coefficients for the Wigley hull models I, II and III show that the larger the water plane area is, namely Wigley II, the more significant the waterline integral effect is. In this case, the free surface condition represented by the line integral, can neither be linearized nor be split into steady and unsteady conditions. They compared the calculated hydrodynamic coefficients to the experimental results by Journée (1992). They reported that their 3D forward speed method including the exact line integral can successfully be used to obtain the forward speed hydrodynamic coefficients of surface ships whose Length/Breadth ratios are greater than 5 when the Froude number is not much greater than approximately 0.3 .

\section{Hydroelasticity methods}

Research continues in improvements to frequency domain methods, as well as 3D time domain methods, the latter, by and large, involving coupling between linear and nonlinear BEM and FE analysis. Coupling between RANS and FE solvers is investigated for slamming related problems (e.g. Piro \& Maki, 2013).

Das \& Cheung (2012) presented a directly coupled hydroelastic approach in the frequency domain with a rigorous treatment of the vessel forward speed. The formulation adopts a translating coordinate system with the free surface boundary conditions accounting for the double-body flow around the vessel and the radiation condition taking into account the Doppler shift of the scattered waves. A BEM, based on the Rankine source formulation, describes the potential flow and the hydrodynamic pressure on the vessel. A FE model relates the vessel response to the hydrodynamic pressure through a kinematic and a dynamic boundary condition on the wetted hull surface. This direct coupling of the structural and hydrodynamic systems leads to an equation of motion in terms of the nodal displacement of the finite elements. The results are compared against predictions from a seakeeping model with forward speed and the modal superposition method at zero speed. A parametric study of a Wigley hull shows that forward speed introduces new resonance modes, which amplify the elastic and rigid body responses of the vessel in waves.

Kashiwagi \& Hara (2012) presented a 3D analysis method for ship hydroelastic problems in the frequency domain, which combines the Rankine panel method for analyzing hydrodynamic responses and the mode superposition method with 3D FEM for representing the structural deformation. The Rankine panel method takes into account the forward speed and 3D effects in a rational way and also the effect of steady disturbance flow on the free surface boundary condition. The natural frequencies of the $d r y$ elastic modes and corresponding mode shapes are computed by a 3D FE method, and the amplitudes of these elastic modes are determined by solving the coupled equations of motion. Numerical results are presented for a $2 \mathrm{~m}$ modified Wigley model travelling in regular head waves, verifying expected performance of the developed calculation method.

Based on beam theory, Miao et al. (2012) developed a methodology for antisymmetric dynamic behavior including, warping function as independent deformation and the influence of structural discontinuity for open-deck ships using a range of theoretical models. The methodology was applied to investigate the response of a container ship travelling in regular oblique waves. The predicted results, in terms of dry hull characteristics and wave-induced loads, are compared with predictions using 3D linear hydroelasticity analysis. It was stated that, although 
the predictions from the $2 \mathrm{D}$ antisymmetric analysis are dependent on the theoretical model adopted, reasonably good agreement was achieved between 2D and 3D hydroelastic predictions; thus justifying their use as a more computationally efficient alternative to 3D models.

Senjanović et al. (2012) presented an improved method of ship hydroelasticity analysis, based on the modal superposition method and including structural, hydrostatic and hydrodynamic models. A beam model is used for the structure comprising a reliable advanced thin-walled girder theory taking into account shear influence on torsion as well as the contribution of bulkheads and the engine room structure, as structural discontinuities affect the ship hull stiffness. Consistent restoring stiffness is included in the analysis via the hydrostatic model. Added mass and hydrodynamics are determined based on the linear radiation/diffraction theory. Also, the analysis of springing effect on the ship fatigue life is introduced using the combination of the improved hydroelastic model and 3D FE substructure model. It is shown that the improved beam hydroelastic model can be efficiently used in the assessment of stress concentrations of ship structural details. Applicability of the developed theory is shown for the global hydroelastic analysis of a 11400 TEU container ship, including stress concentration determination in the selected structural details.

Vidic Perunovic (2012) presented a finite water depth nonlinear hydroelastic strip theory, based on relative motion, for calculation of a ship's wave-induced vertical bending moment (VBM) in the frequency domain and applied it to an analytical beam and a tanker. An increasing trend in the low frequency part of the VBM was noted with decreasing water depth. When the water depth equaled approximately four times the draught of the vessel, the load response shifted towards lower frequencies, and the peak was significantly affected. For the high-frequency response, the magnitude of the springing peak increases with decreasing water depth, primarily owing to an increase in the added mass of the ship and the wave excitation force. Based on the analysis of the tanker, it was stated that the ship's response in different sea states increases substantially when the water depth is less than approximately five times the draught, both in linear and nonlinear springing calculations, compared with the deep water calculation.

Based on the linear random vibration theory, Papaioannou et al (2013) developed a framework for stochastic hydroelastic analysis of very large floating structures (VLFS) subjected to multidirectional irregular waves defined through a directional wave spectrum. The approach involves a discrete evaluation of the relevant transfer matrices through a numerical solution of the fluid-structure interaction (FSI) problem that combines BEM for the fluid potential and the FE method based on the Mindlin plate theory for the plate response. Spectra of responses are obtained as well as extreme responses, assuming a Gaussian input. The proposed method is applied to the stochastic analysis of a VLFS and the influence of the mean wave angle on the standard deviation and extreme values of response quantities is demonstrated. It is found that the hydroelastic behavior of VLFS is greatly affected when considering a directional wave spectrum.

Chapchap et al. (2012) investigated the linear 3D radiation problem of a stationary floating flexible structure undergoing forced oscillations in time domain. The method uses a MEL scheme on an unstructured mesh. The Eulerian phase of the MEL scheme is solved using a constant BEM, in which Rankine sources and dipoles are distributed over the boundaries (i.e. walls, free surface and body surface) of the whole domain. Neumann and Dirichlet boundary conditions are enforced. In the Lagrangian phase, the linear version of the kinematic and dynamic boundary conditions are explicitly integrated in time allowing, at the next iteration, Dirichlet boundary conditions to be imposed on the free surface and the evaluation of the time derivatives of the potential function. The unified symmetric, including both motions and distortions, radiation problem is formulated and applied to the Wigley hull, treated as a uniform Euler beam, undergoing forced rigid motions and distortions. Comparisons with 3D frequency domain hydroelasticity predictions indicate that the implemented numerical schemes are working reasonably well.

Kim \& Kim (2012) presented numerical analysis of ship hydroelasticity based on a fully coupled approach of linear BEM and FE method. For the analysis of FSI problems, a partitioned method is applied. The fluid domain surrounding the flexible bodies is solved by a B-spline Rankine panel method and the structures are modelled using a 3D FE method. The two methods are fully coupled in time domain using an implicit iterative scheme. The computed natural frequencies and motion responses of a simple barge and a segmented barge are validated through 
comparisons with experimental and other numerical results. A large containership is also considered in order to investigate the accuracy of the method on real ship application. Based on the computational results, the pros and cons of the approach are discussed, showing a promising capability for complicated FSI problems. Kim et al. (2013a) applied this fully coupled 3D BEMFE method approach to two real ships, namely 6,500 TEU and 10,000 TEU containerships. The amidships VBM for the 10,000 TEU containership travelling at 5 knots in head regular waves is compared with experimental measurements and another numerical prediction in Fig.2. Kim et al. (2012b) dealt with the numerical springing analysis of containerships. Good agreement is observed in linear responses. However, nonlinear springing responses based on weakly nonlinear approach do not show good agreement with the experiments. In addition, fatigue damage is calculated using this numerical method.

Yang et al. (2013) investigated the FSI for slamming impact phenomena and dynamic structural response problem in containerships at an initial design stage using a direct analysis method. Slamming impact pressures and dynamic structural response are studied using a commercial CFD program (STAR-CCM+) and a structure analysis program (ABAQUS), respectively. These two programs are coupled using the co-simulation function of STAR-CCM+, called the oneway coupled scheme of FSI. Numerical simulations are carried out for bow bottom and stern slamming impact loads of a containership in extreme design wave conditions. Also, the one way coupled analysis has been applied to obtain the dynamic structural responses of local structures. Seng et al. (2014) developed a time domain hydroelastic code for evaluating the global responses on flexible vessels by combining an OpenFOAM VOF-based free surface flow solver and a flexible body motion solver in a strongly coupled partitioned FSI scheme. A numerical example shows that it has the potential to accurately predict the global hydroelastic responses of vessels including slamming and springing. Further work in this field is needed to concentrate on more systematic validation studies in random wave systems where whipping and springing responses are likely to occur.

Lee et al. (2012a) investigated nonlinear wave actions and wave induced global loads acting on a large container ship. An analysis procedure was established to determine values of wave induced VBMs considering the effects due to whipping suitable for design application. The analysis for predicting structural capacities has been carried out by computing the ultimate longitudinal strength of the container ship. Furthermore the assessment of the safety against failure due to excessive maximum loads is accomplished in Ultimate Limit States.

Mirciu et al. (2012) investigated the hydroelastic responses of a large LPG carrier in irregular head waves, based on Longuet-Higgins model with second order interference waves spectra. The LPG carrier has a double hull structure with length over all $238.7 \mathrm{~m}$, design speed 17 knots and two loading cases, namely full cargo and ballast. The numerical analysis was carried out using the in-house code DYN, which is based on the 2D linear frequency and nonlinear time domain hydroelasticity theories, the latter using implicit integration procedures for the equations of motion. The resulting numerical hydroelastic linear and nonlinear response include low frequency oscillations response and high frequency vibratory response, taking into account springing and whipping phenomena induced by bottom and side slamming forces. It was shown that the nonlinear hydroelastic analysis could predict the extreme wave induced loads on the LPG hull structure.

In order to achieve safe and reasonable design with reference to whipping response of ultralarge vessel, Kobayakawa et al. (2012) developed a hydroelastic response analysis system which can calculate the stresses, including hydroelastic responses, on actual ship structures. The details of the system were described and validation study of system in regular wave was carried out. It was concluded that the system can simulate ship motions, hydrodynamic pressures and VBMs in regular head wave conditions with sufficient accuracy.

Malenica \& Derbanne (2012) discussed actual tools and methodologies used in the design of Ultra Large Container Ships (ULCS), especially for the hydroelastic phenomena of springing and whipping. It was concluded that the modeling of springing and whipping is still a challenge and that there is no fully satisfactory numerical tool capable of dealing with these issues consistently.

Based on 3Dhydroelasticity theory of ships previously developed, including the effect of fluid compressibility, Zou et al (2012) further incorporated the hydroelastic and sonoelastic analysis methods with the Green's function of the Pekeris ocean hydro-acoustic waveguide model, to 
produce a 3D sonoelastic analysis method for ships in the ocean hydro-acoustic environment. The method was applied to predict the sound radiations of a floating elastic spherical shell excited by a concentrated force and a LNG ship excited by the propeller induced pulsating forces acting on the wetted bottom plate of the stern in a shallow sea environment. The influences of the free surface and the sea bed on the generalized hydrodynamic coefficients, the acoustic pressure distributions in space are illustrated and discussed.

\section{Slamming and whipping}

Impulsive slamming loads and the consequent vibratory response, namely whipping, continue to be the focus of numerous investigations, as commercial fleets face increasingly rough seas due to climate change and tight schedules. The modeling of water impacts and the calculation of whipping response along with their statistical combination with wave loads remain crucial aspects in assessing ship structural strength.

Analysis of the water exit phase in slamming problems has been the focus of many investigations. Korobkin (2013) used a potential flow formulation for describing the loads acting on 2D and axisymmetric floating bodies that suddenly start moving upward at a constant acceleration. The unknown size of the wetted area is determined by the condition that the speed of the contact points is proportional to the local velocity of the flow. This condition provides a nonlinear Abeltype integral equation which is solved explicitly. Predicted hydrodynamic forces are compared with the CFD results for a rigid wedge of deadrise angle $10^{\circ}$ and a circular cylinder which enters the water and then exits by Piro \& Maki (2011) and Tassin et al. (2013). Piro and Maki $(2011,2012)$ investigated the $2 \mathrm{D}$ slamming problem focusing in particular on the exit phase of a rigid wedge of deadrise angle $10^{\circ}$ which lifts at constant acceleration. Their numerical approach for the air-water flow exploits a finite volume solver from the OpenFOAM library and is applied to an ALE form of the N-S equations. This approach allows computing the solution on moving and deforming meshes whereas the free surface is captured with a VOF technique. Conditions of the impact are selected in such a way that the speed of the wedge becomes zero before the wedge is completely wetted. Piro \& Maki (2011) compared the computed slamming force with Wagner and Von Karman predictions, which overestimated and underestimated the slamming peak, respectively, with respect to the CFD approach. On the other hand the exit force time series from the CFD simulations by Piro \& Maki (2012) compared well with the experimental force measured by Tveitnes et al. (2008). Furthermore, Piro \& Maki (2013) coupled their CFD solver to a deformable structure, i.e. an elastic wedge, using a modal representation that was obtained from a FE modelling of the wedge with beam and plate elements. A linearized boundary condition is used on the common interface to eliminate mesh deformation. In the case of a rigid body experiencing constant acceleration, a satisfactory agreement is obtained during water entry with Wagner theory and, during water exit, with the von Karman model. In the general case, accounting also for wedge flexibility, the numerical method is validated by comparing the structural response with the theoretical results by Korobkin et al. (2006) for hydroelastic impact at constant velocity. The hydroelastic effects were found to be important for a wide range of loading conditions, depending on the imposed entry velocity variation, where the rigid quasistatic solution was shown to underpredict the maximum deflection or stress and the rigid dynamic solution may underpredict or overestimate them.

Tassin et al. (2012a, 2013) addressed the 3D problem by solving the 2D problem of a body with time-varying shape (2D $+\mathrm{t}$ approach). Each $2 \mathrm{D}$ problem was studied by using the modified Logvinovich model (MLM) during the entry stage and the von Karman model during the exit stage. This combined MLM-von Karman model was applied to the vertical water entry and exit of the wedge studied by Piro and Maki $(2011,2013)$. It was found that during the entry stage these two methods agree very well. The agreement during the exit stage is in general good; however, the CFD results by Piro and Maki predict a longer duration and a larger force peak of the exit phase than the von Karman method.

Renewed attention to the water impact problem was motivated also by the analysis of aircraft landing on the water surface (ditching problem) as one of the goals of the SMAES FP7 funded project, as reported by Iafrati et al. (2014). The experiments were performed in the new CNRINSEAN facility that was designed for performing water impact tests at the full-scale ditching speeds up to $180 \mathrm{~km} / \mathrm{h}$. The instrumented elastic plates of the fuselage are attached to a nacelle 
which is pushed by a crossbow system along inclined rails ending in the tank water. This is the first facility of its kind ever built for this purpose. Accurate prediction of the negative forces in the rear part of the fuselage during its landing was shown to be important in terms of the aircraft motion and the bending stresses in the fuselage. The ditching problem was also studied by Hua et al. (2011). To simulate the fluid-solid interactions caused by low speed ditching, the authors proposed a 3D dynamical structural model of the full-scale airplane including the wing and the control surfaces but disregarding the plastic deformation during impact. The FE model was coupled to an ALE fluid field model, and the computational model was then solved within LSDYNA nonlinear FE code. No comparisons were given with either experimental data or other numerical methods.

The exact prediction of the wetted area affected by slamming requires some additional assumptions in the approaches based on the solution of the Laplace equation and, in this respect, some authors pointed out a similarity between the local flow close to the separation point in the problem of water exit and the problem of oblique impact with separation, in particular in the cases where there is a relevant horizontal speed. Reinhard et al. (2012a) implemented three different criteria for the rear separation point in blunt body water impacts with large horizontal and freefall vertical speeds. It was shown that the choice of the separation condition significantly changes the contact point limiting the wetted part of the body, the applied loads and then the predicted motion of the body. Reinhard et al. (2012b) imposed Wagner's condition for the rear contact point to achieve a solution for the oblique hydroelastic impact of a plate into the water. It was shown that the bending stresses in the plate may exceed the yield stress but ventilation decreases the bending stresses compared with a non-ventilated plate. Reinhard et al. (2013) described in detail the fully coupled model developed for representing the elastic plate impact onto the free surface of an incompressible fluid. In this paper they assumed that the fluid is attached to the plate from the turnover region to the trailing edge, so that any suction fluid forces contribute to the dynamics of the plate. The structural equations are solved by modal superposition subjected to a hydrodynamic pressure field along the wetted part of the vibrating plate for which an explicit formula is provided. The authors highlighted that if one applies to the elastic plate the pressure determined from the problem of rigid plate impact, then the bending stresses are overestimated. It was confirmed that the shape of the free surface, the hydrodynamic pressure and the flow, are all sensitive to the plate vibrations.

Tassin et al. (2012b) developed a simplified method based on the displacement potential formulation and the BEM for modelling the impact pressure field on 3D bodies of different shapes. Particular attention is devoted to the analysis of the wetted surface and the predicted slamming force is compared with existing results and with a series of impact tests carried out with a hydraulic machine. The application of a desingularized variational numerical method to the vertical hydrodynamic impact problem of axisymmetric bodies is addressed within the so-called generalized von Karman model by Santos et al. (2012). The 3D body penetrating the free surface is represented at each time instant as a double body built on the wetted part alone of the entire body. The method does not account for the description of the free surface pile up, as in the von Karman theory, but added mass contribution to the slamming force can be accurately computed for any 3D axisymmetric shape and were compared with WAMIT in the case of a sphere and an oblate spheroid.

Water entry of sandwich panels was recently studied because of their potential higher efficacy in absorbing slamming impacts with respect to panels made of homogeneous materials. Xiao \& Batra (2012) employed the BEM for computing the loads on several 2D rigid hull sections because previous computations using LS-DYNA software (Das \& Batra, 2011) exhibited fluid penetration at the solid boundaries. The authors compared the slamming pressure predictions with analytical solutions, other BEM results and experiments for straight wedges with deadrise angles ranging between $4^{\circ}$ and $45^{\circ}$ and curved bow sections, obtaining a satisfactory agreement overall. Subsequently Xiao and Batra (2014) coupled this BEM code to the FE solver of a sandwich panel to study the delamination problem during water entry of an elastic wedge with deadrise angles equal to $5^{\circ}, 10^{\circ}$, and $14^{\circ}$, respectively. The sandwich panel was modelled with a layerwise third order shear and normal deformable plate/shell theory. At each time step the velocity and acceleration of the body and its local deformations are updated according to the condition that the hydrodynamic pressure varies within a prescribed tolerance; otherwise iterations in solving the panel-fluid interface equations restart (two-way coupling). In particular, they 
found that the consideration of geometric nonlinearities, namely nonlinear terms in the straindisplacement relations, significantly increase the peak slamming pressure experienced by the panel. The presence of delamination, studied including the cohesive zone model into a third order shear and normal deformable plate/shell theory, reduces the pressure acting on the panel surface. Delamination affects then deformation values compared to the case where the structure behaves linearly. No comparisons are presented against experimental data or other numerical methods.

Abrahamsen \& Faltinsen (2012a, 2013) considered the effect of air pocket entrapment in 2D slamming phenomena caused by a free surface wave impinging a rigid wall. The air pocket during compression due to slamming is represented as an underdamped mass-spring system. They also derived a new analytical formula for the oscillation frequency of the entrapped air pocket, showing good agreement with BEM and sloshing experiments (Abrahamsen \& Faltinsen, 2011). It was shown that the Topliss et al. (1992) formula is not accurate for large air pockets close to the free surface. The differences between the boundary element solution and the analytical formula showed also that the results are sensitive to the assumed shape of the air pocket. Abrahamsen \& Faltinsen (2013) improved the scaling theory proposed by Lundgren (1969) which involved only the pressure amplitude but not the rise time. The proposed approach is based on fitting experimental data relative to pressure time history (Abrahamsen \& Faltinsen, 2011) with a simple mathematical model governed by two parameters, denoted as kinematic parameters.

Lv \& Grenestedt (2013) studied the sensitivity of the response of a hull bottom to a moving slamming load. Several parameters such as bending stiffness or pressure travelling speed were used. Both the structure, modelled as a linear Euler-Bernoulli beam, and the slamming load, using piecewise constant representation, are simplified. This is a 2D one-way coupling model and these assumptions allowed the authors to calculate analytically the dynamic solution without any dependence of the load on the response. The authors pointed out the existence of slam load travelling speeds at which bending moments and deflections are significantly amplified. Kim \& Paik (2013) developed a design formula for predicting permanent deflection of stiffened plates and grillages under impact pressure loads acting uniformly in space and constant in time, i.e. square wave, on the wetted side of the plate. The method is verified by a comparison with nonlinear FE methods of stiffened plate structures under impact loads. The calculated permanent deflection at the center of the plate was slightly overestimated with respect to ANSYS/LSDYNA results. The largest difference, of the order of $10 \%$, was reached in the case of small plate thicknesses. Though the hydrodynamic slamming load is extremely simplified, the conceived expressions allow inclusion of the slamming problem into the structural design process. To calculate the added mass of elastic wedges impacting on the water, the discrete vortex method was proposed by Fu \& Qin (2014). The same geometrical approximation of Wagner's theory is used and the structure is represented by a modal decomposition. The numerical results relating to the added mass matrix in case of small deadrise angles are in good agreement with analytical ones for the rigid wedge or with numerical ones provided by Khabakhpasheva \& Korobkin (2013) for the case with the deadrise angle set to $10^{\circ}$.

Analysis of more complex geometrical configurations has motivated further efforts in implementing sophisticated computational methods. Panciroli et al. $(2012,2013)$ experimentally and numerically analyzed the water slamming of elastic wedges made of composite panels. In both papers the 2D hydrodynamic load was computed using the SPH formulation available in LSDYNA, accounting for hydroelastic effect due to two-way coupling with the FE solver. In the first paper [2012] attention is mainly devoted to assess the calculation of hydrodynamic loads using SPH through comparison with strain responses recorded in free fall impact tests for various panel thickness, deadrise angle and impact velocity values. Results are in acceptable agreement in the initial stage of the water entry in terms of maximum strains, but accuracy suffers when the wetted zone becomes more fragmented at the late impact stages. Panciroli et al. (2013) focus on capturing the hydroelastic effects on stresses in case of large flexibility of the wedge panels. First it is shown that the experimental vertical acceleration of the free falling body is dependent on the wedge configuration, which could be one of these in the impacting part: cantilever plates, simply supported plates and plates clamped on both sides. In the case of wedges with clamped panels, the solution obtained with the FE/SPH coupled model compared well with results available in the open literature in terms of normalized pressure. Subsequently, the sensitivity of the solution with respect to the ratio between the wetting time and the first natural peri- 
od was analyzed and was found to affect significantly the peak pressures. The BEM based on velocity potential and fully nonlinear boundary conditions in the time domain was applied by Sun et al. (2015) to solve the problem of a 2D wedge entering waves with gravity effect. The gravity effect was found to be relevant for the pressure distribution as the impact time becomes comparable to the ratio of the entry speed to the gravity acceleration. At the same time, impacting a wavy surface results in differences to the pressure distribution on the wedge sides depending on the wave height and wavelength. Scolan \& Korobkin $(2012,2013)$ developed a potential flow theory for an elliptic paraboloid entering vertically a liquid surface on which a regular wave propagates. The Wagner problem is posed in terms of the displacement potential for achieving an analytical solution. Comparisons with experiments and the MLM show good agreement in predicting the initial rise of the vertical force at the early impact stage. Moore et al. $(2012,2013)$ extended the Wagner theory for the normal impact of 3D rigid bodies that are nearly parallel to the water surface. They highlighted that the points at which the free surface turns over in the solution of the Wagner model for the oblique impact of a 2D body are directly related to the turnover points in the equivalent normal impact problem. This observation allowed the authors to discuss the limits of applicability of Wagner theory to 2D oblique impact problems. Thus, for a symmetric body profile, they found that free surface elevation can be considered symmetric after proper change of variables, but this property is not transferred to the leading order velocity potential or pressure. Wang \& Guedes Soares (2013) studied the 2D water entry of a bow-flared section, previously used in drop tests by Aarsnes (1996), with different heel angles using an explicit FE code in order to assess the influence of heel on the slamming pressures and forces. The modeling technique of the FSI adopts the explicit FE code LS-DYNA based on a multi-material ALE formulation and a penalty coupling method. The computed vertical force agreed well with BEM predictions irrespective of the heel angle, even if a significantly lower peak value was found at the maximum heel angle of $28.3^{\circ}$. In general, experimental values were found to be equal or lower than the computed ones with important discrepancies for hell angles larger than $10^{\circ}$. Maximum values increased with increasing heel angle and vertical impact speed for all the approaches considered. Pressure time histories were also compared but relative trends showed less clear interpretations. Yang \& Qiu (2012) computed slamming forces on $2 \mathrm{D}$ and $3 \mathrm{D}$ bodies by solving N-S equations with a CIP-based finite difference method. In the case of wedges the numerical predictions agreed well with BEM results and experiments in terms of the pressure coefficient. They also investigated the free fall of a catamaran segment and a finite length cylinder, for which experimental data in terms of penetration velocity and maximum depth are available, respectively. The agreement was acceptable in both cases. A 3D Cartesian cut cell free surface capturing method was developed by Hu et al. (2013) for water entry problems and applied to rigid wedges and the generic Bobber heave type wave energy device. A high resolution Riemann-based finite volume flow solver for the air-water fluid domain was also implemented and results were compared with available experimental data. Nguyen et al. (2014) studied the water impact of various 3D geometries, namely a hemisphere, two cones and a free falling wedge, with an implicit algorithm based on a dual-time pseudo-compressibility method. Flow fields of incompressible viscous fluids were solved using unsteady RANS equations. A second order VOF interface tracking algorithm was developed in a generalized curvilinear coordinate system to track the interface between the two phases in the computational domain. Sensitivity analysis with respect the spatial grid resolution and the time step was performed so as to assess the accuracy of the results. Free surface deformation, pressure coefficients, impact velocities and vertical accelerations during impact are compared with available experimental data and asymptotic theory, showing good agreement at a reasonable computational cost.

Several papers were devoted to including/coupling slamming load predictions into seakeeping codes. Greco \& Lugni (2012) employed the Ochi criterion for identifying the presence of water slams in the time history of the ship motion provided by a 3D numerical solver. The predicted pressures were then compared with experiments in Greco et al. (2012). A more complex approach for violent FSI, combining a potential flow and a N-S solver within a domain decomposition strategy, was then proposed by Greco et al. (2013) for handling slamming and wateron-deck phenomena at an acceptable computational cost. All three papers are discussed in more detail in Section 2. A benchmark study performed by the ISSC 2012 Committee II.1 Dynamic Response was reported by Drummen \& Holtmann (2014) for a 173m RO/RO ferry. Several ap- 
proaches for calculating the slamming loads in head regular waves, namely BEM over 2D sections and RANS solvers provided by ANSYS-CFX, Star-CCM+ and Comet, were considered and their capability for predicting the induced VBM of a flexible structure was compared. These are illustrated in Fig. 3 for the RO/RO ferry travelling at 25 knots in regular head waves. The reference experimental data were provided from seakeeping tests of a scaled segmented model of a long ferry. The method that generally performed better in predicting bow forces and amidships VBM was based on a fully coupled FSI computation based on the Comet RANS solver for fluid dynamics and a Timoshenko beam for structural representation. This paper is discussed in more detail in Section 12.1. Oberhagemann \& El Moctar (2012) carried out numerical investigations on the 10,000 TEU containership of the WILS JIP (see section 8). Their numerical method allowed implicit coupling between CFD (Comet) and FE structural solvers. Results were presented for the containership travelling at 20 knots in, mainly, head regular waves. The investigation explored different CFD grids, between 240k and $1.8 \mathrm{~m}$. The authors showed good agreement between experimental measurements and numerical predictions in regular waves.. Furthermore, the authors conclude that careful grid preparation allows good results to be obtained with relatively coarse grids. Tuitman et al. (2013) pointed out the need of using a nonlinear seakeeping model, including the slamming load computation, for improving the stress analysis when using realistic 3D FE models. Slamming, Froude-Krylov force and hydrostatic loads included nonlinearities, whilst the diffraction and radiation loads were kept linear to reduce the computational effort. This analysis, though disregarding hydroelastic coupling, appears to provide significant improvements in estimating the ultimate stress on structural details compared to linear 3D BEM. A generalized Wagner model was exploited by Kim \& Kim (2012) for the evaluation of slamming loads within a FSI solver, also discussed in Section 3, coupling a 3D Rankine panel method with structural models of different complexity, namely TimoshenkoVlasov beam model and a 3D shell-based FE representation. The generalized Wagner model required dividing the bow part of the hull into 2D strips for which slamming pressures are computed and then applied to the structural models of a 6600 TEU containership and the scaled segmented model of a 10000 TEU ULC. Comparison of the different approaches with experimental data from scaled model experiments showed the code's capability of describing the slamming induced response. A practical procedure for computing the long term distribution of combined wave and whipping bending moments of container ships was proposed by Ćorak et al. (2013), discussed in more detail in Section 12.1.

\section{Green water}

As the wave breaks and overtops the structure, the flow becomes multi-phased and chaotic as a large aerated region is formed in the flow in the vicinity of the structure while water runs up on to the structure. Thus, the so called green water is generated, which may cause significant damage to facilities and equipment on the deck of the platform. In addition, green water can have strong impact on the stability of marine structures depending on the amount of inundation and global momentum transfer. Green water loads occur when an incoming wave significantly exceeds the free board and water rushes onto the deck. Thus, any increase in the frequency of occurrence and severity of hurricanes implies that structures in the ocean are at higher risk of exposure to extreme waves and green water loads.

Determination of green water loads can be categorized into three methods as approximate, numerical and experimental (ISSC, 2012a). Approximate methods are simple and fast but less accurate and can be used at early design stage. Numerical methods are suggested for more accuracy. Although in most numerical studies conditions are more artificial. The major problem with this approach is excessive computational time. Furthermore, it is not yet possible to obtain realistic results without reflection from the boundaries of the domain. Experiments for green water events are still being carried out today. The objective of such experiments can either be to determine extreme loads or it can be for the validation of numerical methods. Although it is realized that scaled experiments violate scaling laws with respect to the effect of air entrapment, it is still today the best, if not the only, method to arrive at a statistical distribution of extreme loads.

Despite the difficulties associated with analytical research, work in this area continued. Masoud (2013) proposed theoretical calculation of 2D nonlinear wave loads on a horizontal deck of 
a coastal structure located in water of finite depth, based on the Green-Naghdi theory of water waves or Euler's equations. It appears that the girders do not have any influence on the vertical force, and only a small influence on the horizontal force. The effect of formation of air pockets between the girders, in a model of an elevated bridge deck, was studied by adding air pressure relief openings to the deck of the structure. It was found that the entrapment of air pockets increases the vertical uplift force significantly. Results were compared with available laboratory experiments and linear solutions showing a close agreement. This case can be applied to ships with zero forward speed, e.g. moored.

Experimentally, the results of Lee et al. (2012b) for three different FPSO bow shapes in regular head waves were analyzed and compared to each other. The average pressure peaks, for three different wave lengths, where the deck meets a vertical wall are shown in Fig.4, denoting the three different bows by Rect0, Rect5 and Round. Based on these results, a database for CFD code validation was built, such as peak pressure, and some design considerations were proposed. The experiment also shows that pressure peaks were closely correlated with the incoming wave amplitude for each wave length, which can be interpreted as a quadratic relationship between the pressure and velocity of the flooded water on deck. Ariyarathne et al. (2012) examined impact pressure on the deck area of a 3D model structure in a laboratory and related the impact pressure to the measured velocity as well as the void fraction on the deck. Xiao et al. (2014) carried out an experimental study to investigate the wave run-up and green water along the broadside of the 300000 DWT FPSO with a tower yoke mooring system in shallow water, considering non-collinear environments and different water depths. The impact of the motions and the gap between floater and seabed on the relative wave elevations and wave run-up is examined in details. It is shown that the impact of the gap is evident on all the wave elevations along the broadside. Moreover, the impact of vertical motions on the wave elevations along the broadside is relatively small and almost negligible for a large FPSO exposed to extreme waves in shallow water. Buchner \& van den Berg (2013) studied experimentally the nonlinear wave reflection along the side of ships which can lead to green water on deck for moored and sailing ships, with a simplified test setup of a thin plate at an angle to the wave direction. The observed processes with plunging and spilling breakers close to the plate made clear that linear or second order models will not be able to predict this behavior accurately. It was concluded that higher order methods, that could include wave breaking, or CFD are necessary for a prediction of these important effects. These model tests can be used as important validation material. The experimental and numerical investigations by Greco \& Lugni (2012) and Greco et al. (2012), which combine seakeeping and green water analyses, have already been discussed in Section 2.

$\mathrm{Lu}$ et al. (2012) developed a numerical time domain simulation model with VOF technique to study the green water phenomena and its impact loading on structures. Numerical simulations of green water problems carried out in this study include: (i) green water overtopping a fixed 2D deck, (ii) green water impact on a fixed 3D body without or with a vertical wall on the deck and (iii) green water impact on the deck and deckhouse of a moving FPSO model. Numerical results are compared with experimental measurements for both the water height on the deck and the impact pressure on the front wall of the deckhouse for each case, and show fairly good agreement. Xu (2013) examined the case of an extreme plunging wave impinging onto a horizontal deck, simulated by the enhanced, incompressible, SPH method. A first order density smoothing procedure and a density diffusion term in the continuity equation were introduced to smooth the pressure field. A series of simulations were carried out to verify the stability and accuracy of the numerical model. These include a hydrostatic simulation, a bubble rise problem and a dam break simulation. The benchmark studies showed that the enhanced SPH model developed is able to capture details of complex wave-structure interactions including the flip-through phenomenon. The simulations clearly show how the bifurcation of the impinging crest front could lead to the formation of a strong jet (tongue in 3D) that eventually collapses onto the topside of the deck. The remaining portion propagates forward under the deck, leading to higher pressures on the underside of the deck. These results contribute to shedding more light on the dynamics of green water on the topside of a deck in extreme waves. Zhao et al. (2014d) aimed their investigation on assessing the influence of the DoF of a floating body on the maximum impact pressure due to green water on deck. In experiments the DoF can be adjusted by a heaving rod and a restrained spring connecting the carriage and the guide rail. Their numerical method combines a 2D CIP model and VOF-type tangent of hyperbola for interface capturing/slope weighting 
(THINC/SW) approach. Experimental water surface elevations, body motions and impact pressure were compared with the numerical results for different DoF cases, and the agreement is satisfactory. Green water on deck and impact on the deckhouse is generated by the impingement of a focused wave group on the floating structure. The results show that the peak impact pressure due to green water decreases rapidly with increasing DoF.

Kim et. al. (2013c) introduced hybrid CFD method for modelling and simulation of green water load on deck. Their method involves a combined approach of linear seakeeping and nonlinear CFD analysis, both using 3D modelling of the hull. Two large containerships were considered for analysis, for a range of headings. Damage cases of container carrier in way of breakwater and main supporting members were studied. Relative bow motion is considered as the dominant load parameter (DLP). An equivalent design wave for this DLP is used for CFD simulations, modelled as 5th order Stokes wave. Analysis procedure for the direct calculations of design pressure on breakwaters using advanced CFD method is provided in this paper. Newly proposed coefficients for design pressure are compared with IACS UR S3 and found to be reasonable, though on the higher side.

Ruggeri et al. (2013) evaluated numerically green water events in a box-shaped FPSO structure in regular beam waves for a captive model. The potential flow method WAMIT was used for quick checks, whereas CFD software StarCCM+ was used for detailed flow study. 2D and 3D approaches were modelled in CFD. Free surface elevations amidships were compared with experimental results. It is concluded that the $2 \mathrm{D}$ approach can be used only for few relatively long waves, whereas for the entire wave range, $0.75 \mathrm{~L}_{\mathrm{PP}}$ to $1.5 \mathrm{~L}_{\mathrm{PP}}$ in this paper, the $3 \mathrm{D}$ approach is recommended.

Joga et al. (2014) extended application of CFD methodology to simulation of green water to find the rate of water ingress into open holds of a container vessel. Two CFD solvers, StarCCM+ and ANSYS-CFX, were used to model the full scale ship in waves and the effect of water ingress. Computations were carried out only for beam waves and zero forward speed. Various heading and speed combinations were not considered for comparison with experiments due to higher computational requirement. Significant differences between the water ingress predictions of the two solvers and the experimental results were observed, the latter extrapolated from model scale. STARCCM+ results underpredict the mass flow rate, though relatively close to experimental rates. On the other hand, the mass flow rate predicted by ANSYS CFX is much higher compared to the experiments. However, vessel motions computed using both solvers were in line with experimental results. It is concluded that the differences in water ingress predictions are mainly due to free surface capturing by the VOF method and much refined mesh requirement in the region of open holds of the vessel.

Pakozdi et al. (2014) investigated the viability of a simplified coupled method between a potential theory based green water engineering tool, Kinema3, and the commercial CFD tool Star$\mathrm{CCM}+$. Results from a case study application on a large FPSO are validated against model test data. The case study contains analyses of the FPSO in long-crested regular and irregular waves, both in fixed and moored conditions. Three different headings are included. The approach for modelling green water events uses a Finite Volume VOF method with a complex velocity inlet boundary condition. Thus, the Kinema 3 engineering tool is used to generate simplified spatiotemporal inlet conditions from the relative wave elevation and wave kinematics at the bulwark, based on linear potential theory combined with nonlinear random wave kinematics. The VOF method is then used to model the detailed flow on deck, including impact forces on deck structures; that is to say, that only the deck is modelled in STARCCM+. Kinema3 can also generate simplified estimates for the peak water height, velocity and impact force values, assuming an extended dam-break approach together with a simplified, local 2D deck layout. Comparisons with the Kinema3-STARCCM+ results show an overall fairly good agreement, although flow details on deck can, of course, not be expected to be modeled that well. Comparisons of both aforementioned sets of results to model test data show good agreement for the relative wave height, water height and impact force level, in regular and irregular waves. Detailed time histories, including force rise-time, from the coupled Kinema3-Star-CCM+CFD simulation analysis are quite similar to the measured ones. The CPU time consumption for the coupled simulation is moderate, compared to what would have been for a full CFD simulation. Hence, the simplicity of the simulation setup, the quality of the results and the CPU time efficiency makes this method a viable candidate for industrial use. 
Considering the new offshore frontiers for oil exploration and production, in particular the Santos Basin region, FPSOs will be exposed to more severe wave conditions. This scenario requires more careful analysis with reference to the green water phenomenon. The complex physics involved in the water-on-deck flow implies several uncertainties regarding green water load analysis. Carvalho \& Rossi (2014) proposed a methodology, taking into account model tests, CFD simulations and analytical formulations, to estimate the green water loads considering the water elevation above deck measured from experiments or numerical tools. In order to accomplish this objective, CFD simulations with different solvers were run for a benchmark case, showing that it is a suitable approach for a global result in dam-break cases. The term global is used with reference to the pressure computed on block due to the dam break. Other results include wave elevation at various locations. The CFD codes used were: ANSYS CFX, ANSYS Fluent, StarCCM+, Edge CFD and Petrodem MPS. The exploratory CFD benchmark case results showed that this technique is appropriate for complex free surface flows. Comparisons were carried out with experimental measurements by Kleefsman (2005). Although a more comprehensive and systematic evaluation should be done with respect to the differences between experiments and simulations, the simulation results reproduced the most critical pressure time series with approximately 5\% deviations. Furthermore, CFD simulations using ANSYS CFX were carried out to simulate green water events for a typical Santos basin FPSO, together with model tests. Using a combined approach with experiments, a special boundary condition was calibrated to reproduce critical green water events for beam sea condition. In this special boundary condition the inlet surface boundary condition was switched to an open boundary at a particular time. This was done so as to avoid water accumulation in the deck area. Such calibration of the model allows for a detailed flow analysis. Considering the promising results from the benchmark case, this strategy can be used effectively in determining green water loads. Based on a critical event, it was found that the classical dam-break approximation for green water analysis can be very useful for estimating loads on structures, even in complex cases where appendages, in this case the riser balcony, have a clear influence on the flow. In order to extend this formulation to an even wider range of situations, the correction for objects having a vertical gap with respect to the platform deck was verified against CFD simulations, showing good agreement and including a safe margin. The new formulation is then combined with the local water elevation measurements, resulting in a green water loads evaluation procedure. The load reduction of vane type protection barriers is addressed through CFD simulations and, after a parametric study, the effectiveness of each barrier configuration is estimated. For the most efficient protection arrangement, the final load was one third of the original load.

Schiller et al. (2014a) addressed a significant green water problem in the Santos Basin using Kinema3, which is a simplified, yet robust, prediction tool of green water and wave impact on FPSOs in steep nonlinear irregular waves. Given the multi-directional wave conditions in the Santos Basin, the authors expanded this tool's capabilities to consider arbitrary wave directions. Their study case was the FPSO-BR model provided by Petrobras. Predictions were validated against results from model tests performed at Marintek. A good agreement between the Kinema3 green water predictions and the model test records, especially for head seas was observed. For quarter-beam and beam seas, a satisfactory agreement was obtained with an adjustment of the empirical factor $e_{f}$, which is a small non-linear contribution to the relative wave height that is related to the amplification of the wave elevation due to interactions with the vessel. For quarter-beam and beam seas, a lower $\mathrm{e}_{\mathrm{f}}$ factor is necessary, which can be interpreted as wave dissipation processes. For beam seas, the low value of ef is also partially due to the larger importance of roll motion, which may have a mean value and a low frequency component in reality. A progressive decrease of ef with increasing heading angle was observed. The authors conclude that additional verifications with CFD tools and model tests are necessary in order to clarify the relationship between ef, wave heading and the nonlinear wave-structure interaction. The authors also showed that the application of Kinema3 to arbitrary wave directions was a successful step in tackling new challenges associated with the green water problem. One major challenge in the Santos Basin is the bi-directionality of sea states. Although wave impact from the largest waves, coming from S-SW, may be minimized by heading the vessel towards S-SW, the analysis demonstrated that the vessel may be subject to significant green water from E-SE beam seas. The authors showed that beam seas produced the largest green water problem with respect to occurrence, intensity and FPSO deck area affected by water impact. 


\section{Sloshing}

Sloshing is the motion of free liquid surface inside a container and of practical importance with regard to the safety of relevant transportation systems. So far, many researchers have devoted their efforts to studying liquid sloshing problems. Over the course of the reporting period, newly proposed analytical methods, designed experimental setups and developed numerical schemes have been applied to improve the understanding of the sloshing phenomena. Due to the growing LNG market, hence, increasing size of LNG ships and tanks, sloshing has become of increasing concern for designers. Large impact loads induced by sloshing on tank walls may lead to structural damage and also have effects on ship motions and loads.

\subsection{Analytical and numerical methods}

Based on their previous work, Faltinsen \& Timokha (2012) derived a new Trefftz representation for the sloshing velocity potential for the liquid sloshing problem in a $2 \mathrm{D}$ circular tank. This solution included a modified Poisson integral depending on the fully continuous component of the strength functions and terms that are proportional to the jumps of the strength function at the tank top. This work provides an analytical presentation for the sloshing velocity potential that is applicable for higher tank fillings and has a clear mathematical and physical treatment for the linear and nonlinear cases. Numerical experiments with the present solution using different base functions were conducted. Agreement with others' published results is good, suggesting that this solution can approximate the natural sloshing modes accurately for both lower and higher tank filling levels.

Ikeda et al. (2012) studied nonlinear liquid sloshing in a 3D square tank subjected to oblique horizontal excitation with an improved model. In their theoretical analysis, besides the two predominant sloshing modes, five higher sloshing modes were considered by applying Galerkin's method to derive the modal equations of motion. Linear viscous terms were incorporated to consider the damping effects. Van der Pol's method was applied to determine the frequency response curves for sloshing. Experiments were conducted with various excitation frequencies and excitation angles. The quantitative agreement between the theoretical results and the experimental data confirms the validity of the theoretical analysis. Since damping effects were taken into account in their study the obtained frequency response curves, containing both stable and unstable steady state solutions, for amplitudes and phase angles can clearly explain the phenomena of nonlinear sloshing dynamics in real systems. In their study, it was also concluded that higher sloshing modes play a significant role in improving the accuracy of the frequency response curves.

To theoretically study sloshing phenomena in a 2D rectangular tank in shallow water, i.e. low filling conditions, Antuono et al. (2012) proposed a modal system starting with a set of Boussinesq-type equations with a linearized dispersive term. Based on a spatial Fourier decomposition and capable of representing a generic $2 \mathrm{D}$ motion of the tank, this system employs a spatial modulating term to include the exciting forces. This leads to a formulation that is consistent with the conservation of momentum and energy of the flow. Comparisons between experimental measurements and numerical simulations validate the capability of the proposed model. The authors also pointed out the validation of the proposed model confirms that depth-averaged equations can provide a good description of sloshing motions when the water depth is shallow and the waves are non-breaking.

Faltinsen \& Timokha (2013) were the first to construct an analytical method of nonlinear sloshing in a spherical tank. Using analytically approximate natural sloshing modes and curvilinear spatial coordinates, (i) general fully nonlinear modal equations, (ii) weakly nonlinear modal equations and (iii) Moiseev-Narimanov modal system were derived, respectively, for the spherical shape. In their study, the three systems play different roles. The first one is of auxiliary character while the second can be considered as a basis for the third system. The latter was emphasized in this study to construct an asymptotic time-periodic solution and classify the steady state wave regimes appearing as stable and unstable planar waves and swirling for spherical tanks theoretically. The results were compared against experimental data and good agreement was achieved for the liquid depth to radius ratios between 0.2 to 1 . Discrepancies were found for higher ratios (i.e. between 1 to 2) due to the secondary resonance. The formulae for a square- 
based tank developed by Faltinsen et al. (2003) were extended by Takahara \& Kimura (2012) and Takahara et al. (2012), suggesting that the method for the square-based tanks might have uses for appropriate engineering applications.

To model the air pocket impact during sloshing in a 2D rectangular tank, Abrahamsen \& Faltinsen (2012b) used two numerical methods applied at different stages of the simulation. Before the wave touches the roof, a new numerical method called the boundary-element finitedifference method was developed to simulate the two-phase flows. The water domain was discretized by a BEM while the air flow was modelled by a finite-difference method. The interface is tracked in space, satisfying fully nonlinear boundary conditions and remains sharp during the simulation. After the wave touches the roof and the air pocket is entrapped, a MEL method is used. The air pocket is then modelled by a polytropic gas law, whilst the water flow is modelled by a potential flow theory. Comparison between numerical result and experimental data shows that this numerical model overestimates the peak pressure inside the air pocket by $17 \%$ which may be caused by errors in the initial air pocket volume or the 3D effects.

Godderidge et al. (2012a) developed a mathematical model using a phenomenological modelling approach based on the pendulum equation. The equations were numerically solved by a variable-order Adams-Bashforth-Moulton scheme. Damping characteristics were replicated using a first and third order model. The use of modified impact potential makes the model capable of simulating nonlinear sloshing with fluid impacts. Godderidge et al. (2012b) applied this model to evaluate its capability in simulating sloshing in a rectangular tank at critical filling levels. Good agreement with other CFD method showing that the rapid method is sufficiently accurate and suitable for the fast time assessment of sloshing.

Guo et al. (2012b) applied the newly developed finite volume particle (FVP) method based on the fully Lagrangian frame work to simulate the sloshing flow in a 3D circular tank. Four sloshing cases were studied numerically and experimentally. The first two cases are the typical sloshing for a single liquid phase and studied the influence of the circular wall geometry in a sensitivity analysis. The free surface behavior and hydrodynamic pressure are also reported. Sloshing motion and its time of occurrence were compared with experimental data and generally good agreement was observed, while the maximum wave height was overestimated by the present method due to the difference in the initial condition between simulation and experiment. The second two cases simulated the sloshing with solid bodies to preliminary verify the applicability of the FVP method. Good agreement against experiments indicates that the FVP method can be applied to simulate 3D sloshing waves. Recently, Li et al. (2014a) employed a numerical model based on Finite Volume method to simulate sloshing in a 2D rectangular tank with the tree-based adaptive algorithm. The VOF scheme was adopted to capture the free surface. A series of sloshing experiments under roll excitation with various excitation frequencies and filling levels were carried out, obtaining pressure measurements to validate the numerical model. During their experiments, they found that the $3 \mathrm{D}$ effect was more sensitive to the increase of the frequency than the filling levels. They also reported that in lower excitation frequencies cases, standing wave dominates whilst travelling waves are barely found. In all cases investigated the numerical predictions match the experimental data fairy well.

SPH is a popular mesh-free, Lagrangian particle method with attractive features in modelling free surface flows. Shao et al. (2012) used a re-normalization approach to approximate density and applied a corrective kernel gradient to achieve better accuracy with smooth pressure field. Their method is aimed at simulating incompressible liquid sloshing, using the artificial compressibility technique. They also incorporated the RANS model to consider the turbulence effects. A coupled dynamic solid boundary treatment (SBT) algorithm was proposed to improve the accuracy near the solid boundary. Three sloshing case excited considering horizontal and roll excitations, both without baffles, and horizontal excitations with baffles in a rectangular tank were simulated, for a range of filling levels. Good agreement between the present SPH model and the experimental measurements without baffles and other numerical predictions validates the effectiveness of this numerical model. Based on this SBT algorithm, Chen et al. (2013a) proposed further improvements to the SPH model by applying a smaller acting distance of repulsive boundary particles. They also utilized Moving Least Squares method in the density re-initialization to obtain better pressure field. Based on the improved SBT, a new pressure measurement method on solid walls was also given, which took a pressure correction term into account. A series of experiments were carried out using a 2D square tank with $30 \%$ filling levels 
excited by sinusoidal roll motions with four different excitation frequencies. The numerical predictions matched the experimental data well and improved pressure results were observed with this numerical model showing less fluctuations, as illustrated in Fig.5 where the frequency of $3.09 \mathrm{rad} / \mathrm{s}$ is 0.65 of the natural frequency. Recently, Gotoh et al. (2014) proposed two schemes to improve the incompressible SPH-based methods used in the simulation of 2D violent sloshing flows with a particular focus on sloshing induced impact pressure. They comprised a higher order Laplacian of Poisson Pressure Equation (PPE) and an Error Compensating Source of PPE to minimize the instantaneous and accumulative projection-based errors. According to the authors' discussion, there are two error sources in this scheme which are related to the instantaneous variation of particle density and the deviation of particle density from the theoretical constant at a certain time step respectively. Comparison of predictions with available experimental measurements for sloshing in a rectangular tank excited by sinusoidal sway and roll motions, $16 \%$ and $33 \%$ filling levels, respectively, showed excellent agreement emphasizing the enhancing effects of the proposed numerical model. In their study, the importance of dynamically adjusted coefficients of error minimizing terms in the source term of PPE was highlighted, which is shown to be superior relative to a scheme with constant coefficients.

\subsection{Sloshing with internal suppressing structures}

In recent studies, baffles are the most used devices to restrain the sloshing motions. Wu et al. (2012a) developed a 2D numerical model to study the viscous fluid sloshing in tanks with baffles. The sloshing motion is captured by the time independent finite difference scheme while to simulate the baffles, the fictitious cell approach is applied. With the help of the stretch technique of the grid system, the local phenomena of the flow motions around the baffle tip can be captured. An experimental investigation of liquid sloshing in a rectangular tank with baffles was carried out, for surge motion and filling levels of $50 \%$ and $25 \%$. Good agreement between the wave elevation measurements and the numerical results indicate that their present numerical model can be applied to analyze the sloshing motion in 2D tanks with internal structures. Jung et al. (2012) investigated the effect of the vertical baffle height on liquid sloshing in a 3D rectangular tank, 70\% filling level. The ANSYS FLUENT CFD code was applied solve the N-S equations and the VOF method is adopted to track the sloshing motion. In their study, it was found that the critical ratio of the baffle height to the initial liquid height is 0.3 , beyond which the liquid cannot reach the roof of the tank. When the ratio is 0.9 , minimized sloshing wave was obtained. When the ratio is larger than 1, the liquid could not go over the baffle and almost linear behavior was observed. To examine the effectiveness of various baffle arrangements and provide test data for the numerical validation, Akyildiz et al. (2013) designed an experimental setup to study the sloshing behavior with and without baffles in a cylindrical tank with various filling levels under sinusoidal roll motion. In their study, it was reported that ring baffle arrangements are very effective in reducing the sloshing loads. By contrast, Koh et al. (2013) developed a constrained floating baffle (CFB). An improved consistent particle method (CPM) which applies a combination of a zero-density-variation condition and a velocity-divergence-free condition was employed to simulate the sloshing in a 2D tank with CFB. Experiments, in surge excitation, were also conducted and good agreement with the numerical result validated the improved CPM. In addition, it was also found that the CFB was effective in suppressing sloshing motions.

Apart from baffles, perforated screens are also commonly used sloshing-suppressing devices. Molin \& Remy (2013) conducted an extensive series of experiments to study the sloshing motion in a rectangular tank subjected to forced sway and rolling motions with a perforated screen, 53\% filling level for both. In contrast to the experiments carried out by Faltinsen et al. (2011), they focused on measuring hydrodynamic loads by force sensors fitted to the test bench with constant open ratio of the screen and varied motion amplitudes. In their study, damping coefficients were found to be large over a wide frequency range. Thus, they concluded that Tuned Liquid Dampers need not be accurately tuned and can be effective over a wide range of excitation frequencies. Instead of vertical placed screens, Jin et al. (2014) performed experiments to test the effect of a horizontal perforated plate on liquid sloshing in a $2 \mathrm{D} 62.5 \%$ filled tank subjected to sway oscillations with various excitation frequencies and amplitudes. It was observed that the frequency, of the wave generated due to sloshing, was minimally influenced by the plates whilst the wave amplitude was significantly reduced. In addition, they concluded that the 
plates must be placed under and closer to the water surface from the bottom to offer better restraining effects.

\subsection{Sloshing and ship motions}

Wang et al. (2012) investigated the interaction phenomenon of sloshing motions and the global wave loads by seakeeping model tests of a self-propelled LNG ship with a liquid cargo tank, $30 \%$ filling level. It was noted that the existence of liquid in the tank affected the natural rolling period of the ship. The motion period of the liquid in the tank depends not only on the inner shape of the tank and the liquid filling level, but also on the wave heading angle and ship speed. Sloshing induced tri-axial total forces between the tank and the ship are different depending on wave length and travelling speed. The existence of liquid sloshing in cargo tank might reduce the global wave loads of LNG ships to some degree. Sloshing influence from more tanks on global responses of liquid cargo carrying ships in waves need further investigation by experimental and numerical methods.

Mitra et al. (2012) developed a fully coupled model of nonlinear sloshing and ship motion. The fully nonlinear sloshing motion was studied using a FE method, potential flow, and the nonlinear ship motion was simulated using a hybrid marine control system. Simulated roll motions were compared with existing results in waves. Fair agreement indicated that the proposed model was expected to be useful in evaluating the coupling effect. In their study, the coupling model also allowed for the effects of wind, wave and current. It was found that the wave height can have large effects on sloshing, whereas the current velocities caused fairly marginal effects on the ship motions.

Tsai et al. (2013) carried out a numerical seakeeping-sloshing coupling analysis for an 8,000 TEU container ship with some cargo holds partially ballasted, with sea water, to simulate a full load condition, to design draft, required in sea trials. This procedure is unusual compared to the conventional ballast condition in sea trials; the risk induced by resonance of ship motion and sloshing water in cargo holds has to be checked in the design stage. A frequency domain model is utilized in the hydrodynamic numerical code HydroSTAR considering high speed effect, and the analysis includes nonlinear seakeeping, linear sloshing and finally the calculation of the coupled equations of motion. The additional resonant frequencies, especially for the rolling motions, induced by the motion-sloshing coupling effect are clarified and their corresponding operational scatter diagrams, for heading direction change based on specific sea state, are provided. The structural strength of the ballasted cargo holds are to be assessed for those critical rolling motions through a fully consistent quasi-static method by a hydro-structure interaction numerical code HOMER, requiring hydro mesh and FE structure models. These results confirmed that the induced resonant frequency will occur with full load condition and full ship speed, especially for rolling motion, and may cause serious structural problems.

Recently, Zhao et al. (2014c) developed a 2D coupled model which brings together the nonlinear sloshing flows and linear ship motions, both based on potential theory, in time domain. Experiments were conducted to validate the numerical model. An FLNG section comprising an outer rectangular hull and an inner rectangular tank was used in this study. The filling level was $20 \%$ in the tank. The hull was freely floating in the wave tank subject to beam waves. It is similar to past experiments of a barge with a tank on top. Good agreement was achieved for all cases presented. Based on the verified model, the coupling between ship sway motion and internal sloshing was investigated. It was observed that the magnitude of the internal sloshing is nonlinear with the incident wave height, both the internal sloshing and the ship motion exhibited amplitude modulation phenomena and that the sloshing reduction effects on the global motions at the natural frequency are caused by the fact that sloshing force was $\pi$ behind the wave force.

Kim et al. (2014) developed the multi-liquid moving particle semi-implicit (MPS) method. In particular, a robust self-buoyancy model and surface tension model were developed. They also have a new interface searching method for tracing interface particles in a reliable manner. The developed multi-liquid MPS method is validated by comparison with three liquid-sloshing experiments by Molin et al. (2012). The interfacial natural frequencies are simulated by a free decay test using the MPS program and the results are compared with linear potential theory. The simulated results agree well against both experimental and theoretical values. The authors observed that the interfacial elevations can significantly increase when the excitation frequency is close to the natural frequencies of the respective interfaces. Subsequently the verified multi- 
liquid MPS program is coupled to a vessel motion program in time domain. At each time step, the excitations induced by the inner multi-liquid-tank sloshing are fed into the vessel motion simulation and the resulting vessel motion is again fed back into the MPS module. For the case of multiple liquid layers, there exist more than one sloshing natural frequency; hence, the relevant physics can be much more complex compared to the single-liquid-tank case. From the numerical simulations, the authors noted that the wash tank operation in head wave condition is much more robust than that in beam wave condition. The vessel motions with the three-liquid cargo and the corresponding solid cargo were also compared, and it was observed that the wash tank with liquid cargo can also function as a beneficial anti-rolling device. Based on these comparisons it was concluded that the simple approximation using equivalent rigid cargo and ignoring coupling with liquid sloshing can be unreliable in the design stage. Since maximum vessel motions and maximum interface elevations can be checked directly, the developed vessel motion/multi-liquid-sloshing coupled program can be used as a reliable prediction tool for the safety and operability of a floating system with large multi-liquid tanks.

Paik et al. (2014) presented a new method for determining the design sloshing loads, peak pressure and impulse, for FLNG. They also showed an example to validate the method. Thirty scenarios were selected using the Latin hypercube sampling method and a series of numerical computations were undertaken on FLNG cargo containment systems. Based on the results obtained from the study, they conclude that their method is validated using applied examples; hence, feasible for application to FLNGs. The sloshing probability calculated in the study is based on a limited amount of motion data. Thus, if field measurement data were to be used with this method, the accuracy of the sloshing loads would be more reliable and robust.

\subsection{Experimental investigations}

Ji et al. (2012) studied the nonlinear sloshing experimentally with the aid of the time history of wave elevation at the sidewall, free surface profile captured by high speed camera and velocity vector flow field obtained by PIV technique. In this study, non-resonant sloshing under the deep water filling condition was considered in a harmonically oscillating (surge) rectangular tank with breadth of $60 \mathrm{~mm}$, length of $300 \mathrm{~mm}$ and height of $180 \mathrm{~mm}$. Based on the experiments, they found that four regimes of sloshing waves can be categorized: mild 2D wave, strongly nonlinear 2D wave with hydraulic jump-like motion, 3D wave with regular structure in the longitudinal direction, and 3D chaotic wave. In their study they focused on a comprehensive discussion on strongly nonlinear 2D wave and they concluded that this off-resonant sloshing problem can be characterized into a combination of three sloshing motions: (i). standing waves during rundown process similar with linear sloshing, (ii) hydraulic jump along the vertical wall during the run-up process and (iii) bore motion propagating from the sidewall to the interior fluid region resembling a dam break during stationary process. The authors presented time histories of wave elevations on the tank wall, wave profiles and velocity vectors at selected time instants to show the evolution. These can be used to validate relevant numerical models.

To measure the impact pressure with accuracy during the sloshing process excited by large sinusoidal sway motion, Pistani \& Thiagarajan (2012) setup a sloshing experiment in a 2D rectangular tank. In their study, the artificial pressure spike acquired by the transducer when measuring in the two-phase environment was resolved by lowering the excitation voltage of the sensors. Details of the analysis of characteristics of the pressure traces during the impact of the fluid and their location in the tank were also discussed, for a $30 \%$ filling level only. In addition, they proposed a strategy for defining a threshold pressure based on the transducer location and sloshing impact time.

Kim et al. (2013b) introduced the sloshing experimental facility at the Seoul National University (SNU). Recently, SNU equipped a new experimental facility for sloshing model testing, and this paper summarizes recent research and experimentation carried out. In particular, this paper focuses on the experimental setup, post-processing of measured signal, and findings from recent sloshing experiments. Their findings, being useful to those engaged in experimentation, are summarized as follows:

- Integrated circuit piezoelectric (ICP) sensors are sensitive to the change of contact medium. Temperature difference between the sensor and contact medium (i.e. gas or liquid) must be minimized before sloshing experiment. Sensors of the same type, diameter and linearity can show 
different results. Therefore, the pressure sensors need to be carefully chosen and applied to sloshing experiment.

- The density ratio of liquid and gas should be carefully handled in sloshing experiment sand analysis. The present experiment showed generally higher sloshing impact pressure with the higher density ratio of gas and liquid applied. Thus, when sloshing model test is performed, the consideration of the density ratio of liquid (LNG) and gas (NG) which satisfies that of actual LNG cargo is recommended in model tank.

- The current pressure-visual synchronized system may provide some important correlation between impact pressure and instantaneous flow velocity. The current PIV system showed oscillatory velocity field during sloshing impact occurrence. This type of observation is helpful for the development of numerical simulation code and understanding the relationship between kinematics and dynamics during sloshing.

- Statistical properties of peak pressure are dependent on the size of the time window in sloshing experiments for both regular and irregular motion excitations. In the case of sloshing experiments at SNU, regular excitation required about 500 cycles for reasonable convergence and about 50-hour motion excitations were suitable for irregular motion.

Zhao et al. (2014b) carried out scaled model tests for an FLNG section excited in roll by white noise waves. Comparison studies of the vessel motions using fresh water and equivalent steel ballast weights are conducted. The following conclusions are drawn from this experimental investigation: (i) the spectral peak of the roll motions shifts to a lower frequency due to the changed center of gravity and the moment of inertia for the reference filling conditions in the study; a spectral peak of the internal sloshing oscillations is observed at the corresponding excitation frequency; (ii) sloshing at the head and the following sides of the inner tank exhibits similar responses for the reference configuration in this study; (iii) prominent RAO peaks of the internal sloshing oscillation have be observed for the excitation frequencies that are equal to the natural frequencies of sloshing in different modes; (iv) a large RAO of the internal sloshing at some excitation frequencies does not necessarily correspond to a large RAO of the vessel motions. Only one filling condition was selected as the reference. Therefore, great care is required when extending this study's conclusions directly to cases of translational motions and other filling levels.

\section{Loads from abnormal waves}

Research on abnormal waves and their consequences on marine structures continue on many fronts, mainly focusing in new techniques of experimental generation of abnormal waves, experimentation of abnormal wave-structure interactions and numerical modelling to assess the adequacy of the methods used in representing the abnormal wave and its interaction with marine structures.

Bennett et al. (2012a) investigated how best to generate accurate, repeatable and controllable unidirectional abnormal waves in an experimental test facility for the purpose of practically modelling the response of a travelling vessel to abnormal waves. Three techniques, based on linear superposition principles, for producing an abnormal wave from a sea spectrum were compared, namely NewWave, Constrained NewWave and Optimized Sea. Each technique was tested for multiple sea spectra. The experimentally generated waves were compared to both linear and second order wave theory, to assess the adequacy of the former as it is used in the numerical models for predicting ships response and it is important to ensure they replicate the abnormal wave record used during experiments. The advantages and disadvantages of each abnormal wave generation technique are summarized in the paper. Results showed that in order to allow the most realistic predictions of ship responses, inclusion of the response history of the vessel, hence the random sea surrounding the abnormal wave is required, i.e. embedding the abnormal wave in an irregular wave. Two of the techniques used allow this, namely Constrained NewWave and Optimized Sea. The latter is the preferred option for realistic predictions, as it offers more control over the abnormal wave shape and significant anomalies between the required wave and that generated in the tank were observed for the Constrained NewWave technique.

Onorato et al. (2013) used a breather solution based on the one-dimensional nonlinear Schrodinger equation to generate critical wave sequences containing abnormal waves in the tank of the Technical University of Berlin. A breather solution describes the modulation of a slightly perturbed wave and is considered as a suitable modelling tool for abnormal waves. The seakeep- 
ing tests were carried out using a $2.3 \mathrm{~m}$ model of a chemical tanker. The investigators carried out measurements with and without the model showing that the presence of ship did not alter the measurements of the generated wave. They also obtained satisfactory agreement between theoretical location of freak wave and tank observations. The amidships VBM and water-on-deck were amongst the measurements of interest for this committee. To measure the former the model comprised of two segments connected with force transducers. A large VBM was measured during the impact of the abnormal wave, exceeding what the authors referred to as early stage design VMB, especially in the sagging condition by approximately $40 \%$. Furthermore, the water gauge on deck measured a water column of $10 \mathrm{~m}$ full-scale during the impact. Although there are traces of high frequency components in the VBM record shown in this paper, it is difficult to draw any conclusions with reference to their origin. Nevertheless, this work emphasizes that abnormal waves have a significant influence not only on global loads but local loads such as water on deck.

Bennett et al. (2013) investigated experimentally the influence of forward speed on the motions experienced by a frigate encountering an abnormal wave and the implications that such encounters may have for ship design. Long-crested abnormal waves were generated using the NewWave and Optimized Sea techniques, thus allowing the influence of vessel response history on ship motions to be investigated. Random irregular waves, not exhibiting abnormal wave characteristics, were also used as comparators. The height of the abnormal wave tested in was defined relative to the sea state using the abnormality index and relative to the encountering vessel using the length index developed during the course of this research. Motion measurements are compared to prediction using 2D linear strip theory, and 3D partly nonlinear seakeeping theory, both showing good overall agreement and the latter showing better agreement with increasing forward and wave height. The severity of motions experienced by the frigate in abnormal waves increases with forward speed, until the frigate appears to be tunneling through the abnormal wave. Response history is a dominant contributor to ship motions, particularly at higher speeds and it is, therefore, essential to include it. The unexpected nature of abnormal wave occurrence means that it is important to consider additional factors such as likely operating condition and ship speed at the time of encounter when considering implications for ship design.

Vasquez et al. (2013) carried out experimental and numerical investigations on a bulk carrier stationary and travelling $(\mathrm{Fn}=0.1)$ in irregular head waves with embedded abnormal waves. Two abnormal waves were used based on existing records, e.g. the new year wave. A segmented model, with the segments connected by force transducers, was used for the experiments and heave and pitch motions and amidships VBM were recorded. The numerical predictions were obtained using a 2D rigid body time domain method, including nonlinear hydrostatic and incident wave contributions, whilst the radiation component is based on the mean wetted surface hydrodynamics in the frequency domain and accounted for through IRFs. In addition the effect of green water, but not that of slamming, is included. The authors noted that the nonlinear effects in the numerical predictions were small, attributed to the hull shape of the bulk carrier. The overall agreement between experimental measurements and numerical predictions is good for $\mathrm{Fn}=0$, but $\mathrm{VBM}$ is overestimated for $\mathrm{Fn}=0.1$, especially for the more extreme wave condition. The authors observed that for the bulk carrier tested the influence of green water on the global loads was not important. With reference to the effects of forward speed a maximum increase of $27 \%$ for the VBM was observed for the more extreme case, although it is difficult to draw conclusions as only one non-zero forward speed case was tested.

Bennett et al. (2012b) compared experimental measurements of rigid body motions and global wave-induced loads of a naval frigate in abnormal waves to predictions made using a 2D linear hydroelasticity method. Experiments are conducted using a segmented, flexible backbone model of a typical naval frigate constructed of four rigid segments attached to a uniform aluminum backbone. The model was designed to match the natural frequency of the 2-node vertical bending moment. VBMs were measured at midships and the aft and stern quarters. The 2D linear hydroelasticity model idealized the vessel as a non-uniform Timoshenko beam and slamming was included via the Ochi-Motter impact slamming model. Qualitative agreement is seen between experimental measurements and 2D linear hydroelasticity predictions of VBM in abnormal sea states. However, the numerical predictions in general overpredict the maximum VBMs experienced. It is thought that this may be due to no consideration being given to the nonlinear 
effects when evaluating steady state components and future work should consider this. Furthermore, the maximum VBM along the vessel occurs at the aft quarter in experiments and amidships in predictions.

Zhao \& $\mathrm{Hu}$ (2012) carried out experimental and numerical investigations on a 2D box shaped body, with a superstructure for green water simulation, in abnormal waves, focused and combined with a regular wave. The aim of their investigation is to assess how the RANS CIP (Constrained Interpolation Profile) -based method performs with extreme waves, ignoring turbulence and surface tension effects. There is good agreement between numerical and experimental wave profiles. Motions in the vertical plane, heave and pitch, and pressure on deck were measured in the experiments, with the longitudinal displacement constrained. Good agreement between predicted motions and measurements is obtained for the focused wave, but the predictions are smaller than the measurements in the more realistic case of the combined wave. The method also underestimates the pressures on the deck, but the overall qualitative agreement between predictions and measurements is satisfactory, showing that the method in question is suitable in modelling highly nonlinear phenomena. Zhao et al. (2014a) continued these investigations by refining their $2 \mathrm{D}$ numerical model by incorporating a more accurate free surface capturing method based on VOF. They also allowed for the surge motion, both in the experiments and numerical investigations, comparing the influence of constraining this motion. Only the focused wave was used in this work. When the surge is constrained, the agreement between experimental and numerical predictions for the motions shows some improvement, especially for the pitch motion. In the case when the box is free to surge, there is underestimation of the pitch motion attributed by the authors to the increasing complexity of the flow. With reference to the pressure on deck, the improved free surface capturing results in small improvements in the predictions. Nevertheless, the pressure is underestimated, when surge is either constrained or not, by comparison to the experimental measurements. The authors also investigated the influence of grid density on their predictions using coarse, medium and fine grids. The effect of grid density is small on the motions with the coarse grid providing closer predictions to the measurements. The on-deck pressure predictions are influenced more by the grid density, with the middle grid showing the best overall agreement, still underestimating, and the fine grid having issues with the first pressure peak when surge is not constrained.

$\mathrm{Hu}$ et al. (2014) investigated the interactions between a freak wave and a beamlike structure using 2D numerical modelling, equivalent to assuming that the beamlike structure is infinitely long in the athwartships direction. Nevertheless, their investigations are of interest due to (i) coupling between a RANS (VOF) solver and (linear) FE method, through iterating the fluid pressure field and velocity and deformation of the structure at each time step; (ii) the successful generation of a, long-crested, freak wave based on real measurements; and (iii) the use of different boundary conditions for the beam, including a rigid beam. There are no comparisons of the predictions with either experimental measurements or other numerical predictions. Their observation that the beam deformation affects the bottom impact pressure, in the sense of pressure reducing with increasing deformation, is of interest. They also observed that there were relatively small differences between dry and wet natural frequencies, probably due to the $2 \mathrm{D}$ simplification of their modelling approach.

With ship encounters with abnormal waves occurring more frequently, it is important to assess whether such encounters are causing ships to operate beyond the limits of the design rules. Bennett et al. (2014) experimentally assessed the global loads experienced by a typical naval frigate in a range of abnormal sea states generated using the Optimized Sea technique. Experiments are conducted using a segmented, flexible backbone model (Bennet et al., 2012b) operating at its service speed of 18 knots full scale, in head seas. Measured VBM values are compared to those obtained from the design rules for naval vessels (Lloyd's Register, Bureau Veritas and Det Norske Veritas), both with and without allowances for extreme wave events. The presence of an abnormal wave event in a sea state increases the maximum VBM the frigate experiences by up to 2.5 times compared to in an equivalent random sea. The severity of slams experienced by the ship increases significantly with $40-60 \%$ of slams having a forefoot emergence of greater than $25 \%$ the ship length. Fig. 6 presents the percentage of slams occurring at different severities of slamming length (or forefoot emergence, defined as a percentage of the ship's length) in random and abnormal (or optimized) waves, in three sea states corresponding to South Atlantic, North Atlantic and Southern Ocean conditions. The distribution of slams in random seas appears 
more even across the range of slamming lengths. $40-60 \%$ of slams in abnormal seas occur with a forefoot emergence of $25 \% \mathrm{LOA}$, compared to $15-35 \%$ in random seas. This demonstrates that the presence of an abnormal wave greatly increases the number of severe slams occurring. The authors recommend that, in order to have a sufficient safety margin, design rules that account for extreme waves should be used; however, there were still some cases where this did not appear sufficient and these require further investigation.

Likelihood of occurrence of abnormal waves in relation to recent climate changes is an extremely important subject area affecting the consideration of abnormal waves when evaluating design loads. Accordingly two fairly recent papers are singled out for inclusion in this report. Vanem et al (2014) dealt with uncertainties in the climate modeling and the effects of abnormal waves on the wave bending moments of ships. This may significantly affect the safety level of existing and newly developed ships; thus, it is an important area of concern. The uncertainties in the modeling of both the climate changes and in the modeling of the wave statistics are significant and conclusions vague. Without claiming generality, when comparing historical with simulated data, surprisingly the statistical mean and up to the $90 \%$ statistical quintiles of the historical data of wave heights are higher than the simulated future data for some selected scenarios. Also, the standard deviations are larger for the future projections, whilst the statistical upper tails are longer. The interpretation of this behavior and the consequences/conclusions on the way ahead are very crucial. In a further paper by the same research team, Bitner-Gregersen et al. (2014) presented the impact of climate change and extreme waves on tanker design. The study indicates that observed and projected changes in wave climate will have large impact on tanker design practice. Necessary increase in partial safety factor(s) and/or revised specification of the characteristic wave bending moment need to be further investigated.

\section{Experimental and full scale measurements for wave-induced loads}

In the last few decades, the investigation of complex FSI problems related to slamming phenomena has motivated not only the need for reliable simulation, but also more challenging objectives for experiments in model basins and for sea trials. In this section, therefore, recent research aimed at studying wave-induced high frequency response by means of experimental tests and full scale observation is discussed. Measurements related to sloshing and green water were reviewed in Sections 5 and 6, respectively.

Lavroff et al. (2013) carried out an extensive experimental investigation of wet-deck slamming in regular head waves using an elastically scaled model of a wave-piercer high speed catamaran. The segmented model consisted of three parts with the forward part comprising the forward demi-hulls and the center bow as a separate segment. The sophisticated design of the catamaran model allowed measuring the slamming vertical force acting on the center bow and the vertical bending moment along the demi-hulls in order to identify the critical conditions in the operational envelope of the vessel. Slamming occurred in a range of the encounter frequency that was found to be relatively larger in the case of the higher regular wave amplitudes and the induced loads were approximately proportional to the wave amplitude squared. The longitudinal position of the slam impact on the center bow showed a small but significant dependence on the wave height and frequency. The peak slam load measured on the centre of the bow, as a function of dimensionless encounter frequency, is shown in Fig. 7(a) and the corresponding demi-hull peak VBM at the forward segment position in Fig.7(b), the latter being the total measured in the forward and aft segment hinges. Comparing figs. 7(a) and 7(b) one clearly notes the similarity in distribution between slamming load and VBM. Wu et al. (2012b) discussed the capability of representing the bending full scale behavior with segmented models made of rigid segments and flexible joints. Several aspects, such as the number of segments and the stiffness distribution, are considered for accomplishing the hydroelastic scaling of a 13000 TEU containership. The authors found that 3-joint segmented structural model provides a sufficiently accurate representation of the real ship, if the hydroelastic effects depend mainly on the first vertical 2-node flexible mode. These hydroelastic effects in the vertical bending moment appeared not to be sensitive to the stiffness distribution among the flexible joints in a 3-joint segmented model. Hence, the authors concluded that the same joint stiffness can be applied when adjusting the model to achieve the targeted natural frequency of the first flexible mode. Zhu and Moan $(2013,2014)$ focused on the wave-induced nonlinear effects which appear in VBM response of ULCS. Zhu 
and Moan (2013) focused on the explanation of the differences between sagging and hogging VBM extreme values. Amongst well known sources of asymmetry, like nonlinearities in the hydrodynamic forces and ship hull geometry, in particular, they considered the nonlinear contribution to the overall loading due to the pressure field acting on the bow which affects mainly the hogging VBM. Two container ships, 8600 TEU and 13000 TEU, were considered with segmented model tests in regular and irregular head waves. Regular waves allowed the authors to clarify the role of super harmonics in increasing or decreasing the hogging and sagging peaks. High, but rather unrealistic, speeds for such ships in rough seas had a significant impact on the VBM asymmetry through higher order harmonics and high frequency vibrations mainly more in sagging than hogging. Zhu and Moan (2014) investigated the effect of regular and irregular oblique waves on extreme values of VBM for the case of a 13000 TEU ULCS. The authors found that the extreme values for both sagging and hogging were higher in oblique waves than in head waves. In particular, the second harmonics of the amidships VBM affected the peaks in regular waves to an extent which showed to be dependent on the heading angle.

Dessi and Ciappi (2013) investigated the slamming occurrence for a ferry cruising at high speeds with an elastic segmented model tested in irregular long-crested waves. They observed that the tendency of the slams grouping into sequences made of two or more events (clustering phenomenon) violated the hypothesis of their statistical independence. Sequences of closely occurring impacts were found to produce higher or lower global responses depending on the phasing with ship-beam oscillation excited by the previous impact(s). The combination of high speeds and high sea states was found to play a relevant role in inducing the slam clustering. However, a direct correlation between each slamming event and the encountered wave height, or even between the slamming severity and the impact velocity, was not always present. Time separation of slams inside a sequence was approximately equal to the natural pitching period of the scaled ship. A new criterion based on the wavelet analysis of the amidships VBM was then introduced to identify the occurrence of a slamming event, apart from the usual distinction between bottom or bow-flare slamming events. A slightly different version using filtering and Hilbert transforms for the analysis of transients is also presented by Dessi (2014a). Jacobi et al. (2014) applied a similar criterion to the identification of slamming events during full scale trials on a wave-piercer high speed catamaran. This criterion is based on the definition of a stress rate threshold, first proposed by Thomas et al. (2003). The choice of the strain gauge location proved to be critical for the correct application of the proposed slamming criterion: the strain gauge located in the centrebow archway and mounted on a stiffener cut out at frame 64 was at the end selected, as it clearly presented vibrations uniquely associated to slamming. To avoid false positive slamming events, the analysis of the stress time history needs to be combined with a second criterion detecting the downwards decelerating bow motion. Using this criterion, the number of slams per hour with a certain intensity can be determined and their dependence on speed, wave height and heading analyzed. The influence of the ride control system in alleviating the slamming stresses was found to be more relevant at high speeds. The existence of an overall trend, with a weak association, between the relative vertical velocities and the stresses occurring after the slam impacts was noted. However, the association is so weak that it cannot be used as a primary indicator of slam occurrence and magnitude, similarly to the case of fast monuhulls as shown by Dessi and Ciappi (2013).

Andersen \& Juncher Jensen (2014) investigated the effect of hydroelastic high frequency vibration on the extreme hogging wave bending recorded onboard a 9400 TEU container carrier. The stress measurements amidships were obtained using two long-base strain gauges mounted in the passageways, port and starboard sides, just below deck approximately amidships. The wave field close to the ship could be estimated with the onboard wave radar and the rigid body response was recorded from an inertial motion unit. They found that the 2-node mode is, by and large, responsible for the ship vibrations even in quartering seas, with no evident torsional contribution, and that the hydroelastic contributions may double the stress levels at some instants. Thus, their analysis brought to attention the possibility that predicted total wave hogging VBM, accounting also for whipping loads, may exceed slightly the design stress values for that ship in extreme but realistic sea states. The combination of steady state wave loading with loads associated to whipping and springing is also analyzed by Barhoumi \& Storhaug (2013) in the case of 8600 TEU container vessel, equipped with a comprehensive onboard hull monitoring system with 20 strain sensors for global and local hull response and monitored for approximately 4 
years. On the basis of the strain data collected at various ship sections, the authors found that the contribution of whipping to the reduction of fatigue life and to extreme loading is important in particular zones of the ship structure. Though fatigue appeared as a less relevant issue for the east Asia routes compared to the north Atlantic ones, the effect of voluntary speed reduction and routing on the structural loads continues to be significant for this type of vessels.

Building on the success of the WILS-I JIP Hong et al. (2012) reported on WILS-II comprising a series of model tests on a 10,000 TEU containership model made up of 6 segments and an elastic $\mathrm{H}$-shaped backbone, allowing both bending and torsion induced springing and whipping to be included. The experiments were carried out both in regular and irregular waves, for headings of $180^{\circ}$ and $150^{\circ}$ and a range of ship speeds. The authors concluded that the H-shaped backbone used was successful in matching both bending and torsional stiffness. Furthermore, Hong \& Kim (2014) reported on phase III of the WILS JIP, on the same containership, using a U-shaped backbone model comprising 6 segments and again allowing both for bending and torsion. This investigation focuses more on higher order response and includes comparisons between $\mathrm{H}$ - and U-shaped backbones.

Iijima et al. (2014) carried out an experimental investigation on the post-ultimate strength collapse behavior of a ship's hull girder under whipping loads. The 1/100 scaled model comprises two rigid bodies, a connecting deck hinge and a sacrificial specimen amidships which bent under the relative rotation of the two segments. The mechanical properties of the model and, in particular, the ultimate bending strength follow the similarity law proposed by Wada et al. (2010). The tests were conducted keeping the model afloat on the water and the whipping loads were artificially reproduced by dropping an object onto the ship section. It was observed that the sacrificial specimen collapsed less when the whipping loads had a shorter duration, indicating that the collapse may not occur to a large extent under impact loads. An original experimental approach of 3D water impact at constant speed was presented by El Malki Alaoui et al. (2012). The hydrodynamic force acting on axisymmetric rigid bodies striking a horizontal liquid surface at constant vertical speed was investigated using a hydraulic shock machine, which allows carrying out impacts at high speeds with small deviations in the velocity. Comparisons between theoretical model, numerical results, also discussed by Tassin et al. (2012b), and available experimental measurements are reported in this paper showing an acceptable agreement.

Dessi (2014b) presented a technique based on a combination of proper orthogonal decomposition and spline approximation for reconstructing the unsteady distribution of sectional loads due to waves along a ship hull. To validate the proposed technique, the hydrodynamic lumped forces acting over hull segments were initially made available by calculating numerically the sectional loads and then spatially integrating these loads over the segment sections. The set of time-varying segment forces constituted the input data from which the original sectional load distribution was successfully reconstructed. The robustness of this technique was tested against the presence of noise in the input data. This approach was used by Dessi (2013a) for obtaining the experimental slamming load distribution in regular wave tests with a segmented hull. The time history of the forces acting on each segment was measured with load cells and the sectional slamming force at each time step was extracted on the hull sections subjected to water impacts. Thus, a greater detail in the spatial load distribution can be obtained from experiments with segmented models and structural ship design can benefit from reliable load data with improved resolution.

Several papers dealt with signal processing techniques for highlighting some features of the ship vibratory response. Kim et al (2013d) proposed the use of the cross correlation between the wavelet transforms of the ship response and an ideal and tailored IRF to extract the whipping part of the response. The authors successfully applied this technique to a realistic signal obtained from the WISH-Flex simulation code. The analysis of the ship vibratory response was also considered by Mariani \& Dessi (2012) for identifying the wet operational modes in terms of shape, frequency and relative energy associated with each elastic mode, with proper orthogonal decomposition which does not require the measurement of the exciting force. They compared seakeeping tests of an elastically scaled model in head waves for different sea states, with/without slamming as the case may be, highlighting how energy is captured mainly by the 2-node vertical bending mode. Damping estimation of the experimental whipping mode using the random decrement technique (RDT) or the autocorrelation function (ACF) was considered in a successive paper by Dessi (2013b), using the same dataset used by Mariani \& Dessi (2012). 
The damping values were found to be different depending on the particular signal processing technique, RDT or ACF, used and on the choice of some parameters within the same technique. The random decrement technique in combination with the wavelet transform was also applied by Kim \& Park (2014) to data obtained from elastic model tests for damping identification of the wet bending modes. The authors explained the differences in modal damping estimation between still water conditions and forward speed in waves with the presence of viscous effects.

Ikeda \& Judge (2014) carried out a series of tow tank experiments performed on a $1.2 \mathrm{~m}$ model hull in regular waves. Pressures on the bottom of the hull were measured on one side with traditional point-pressure sensors and on the other side with pressure mapping pads. The goal of this research is to study the fundamental physics of the water impact of high speed planning hulls and to measure the slamming loads and resulting motions of the craft upon reentry into the water after becoming partially airborne. A set of towed model experiments was conducted in calm water, regular waves and irregular waves to capture a sequence of individual impact events. The pressure signals from the pressure pads (providing both spatial and temporal resolution) and the point-pressure measurements (high temporal resolution) have been presented for individual slam events, allowing a deterministic approach to investigating high speed planing craft wave slamming.

\section{Loads due to damage following collision/grounding}

One area that has become of great concern to the design and operation of ships is that of accidental damage. Accidental damage to ships and, subsequent, flooding can occur in a number of ways, but generally damages due to collision and grounding are of primary concern, particularly for ships at high risk with respect to the loss of lives or pollution of the marine environment. Naturally, events of non-accidental structural failures leading to ship's loss are also of great concern for some ship types, e.g. the loss of containership MOL Comfort in June 2013. The aim of this section of the report is to focus on the load implications on the ship after the event, mainly on loads of hydrodynamic origin and not to replicate part of the work undertaken by Committee V.1 Accidental limit states.

The IACS Harmonized Common Structural Rules (CSR-H) (IACS, 2013), are aimed at checking the hull girder ultimate bending capacity in the damaged state for the seagoing condition to ensure that it satisfies the residual strength checking criteria. Accordingly the VBM, $M_{D}$, in hogging and sagging conditions, to be considered in the ultimate strength check of the hull girder in the damaged state is obtained as:

$$
M_{D}=\gamma_{S D} M_{S W-D}+\gamma_{w D} M_{w v}
$$

where $M_{S W-D}$ is the permissible still water bending moment, $M_{w v}$ is the rule vertical wave bending moment (VWBM), $\gamma_{S D}=1.1$ is the partial safety factor for the still water bending moment SWBM) in the damaged condition and $\gamma_{W D}=0.67$ is the partial safety factor for the VWBM in the damaged condition. The general characteristics of global loads on damaged ships are apparent from the partial safety factors included in Eq.(1). The SWBM may increase with respect to permissible SWBM in intact condition, whilst the rule VWBM is considerably reduced. The most dangerous situation is the flooding of ballast compartments in the amidships region, causing increase of sagging SWBM in the vicinity of the damage. Downes et al. (2007) performed a case study on an Aframax tanker showing that in the full load condition $10 \%$ of the cases lead to an increase in sagging SWBM of $25 \%$ or more of the allowable SWBM. The literature review of increases of SWBM used in structural reliability assessments of damaged oil tankers is presented by Burić et al. (2012), where they found that SWBM in sagging may in some cases increase by a factor of two compared to the intact SWBM.

Differences between wave load effects employed for intact and damaged ships arise because of the effect of flooding on the calculation of hydrodynamic loads, as well as from different environmental conditions and exposure times to be taken into account. Downes et al. (2007) suggested two practical approaches for calculation of hydrodynamic loads on a damaged ship. The first is the added mass approach where the seawater which floods into the vessel is assumed to become part of the vessel's mass and moves with the vessel. For calculating the hydrodynamic forces, the damage opening is assumed to have negligible impact on the overall hydrodynamic 
properties of the hull. This approach should be accurate for damage extents which are small compared to the size of the tanks which are breached. The second approach, the lost buoyancy method, is applicable for larger breaches, where the damaged tank and all of its mass are removed from the vessel, as well as its surface area from the hydrodynamic model. The hydrodynamic interaction between the waves and the structure of the opening needs to be modelled. Comparison of transfer functions of linear VBM at amidships calculated by two methods for damage modelling and for intact ship is presented in Fig. 8. Results are obtained using 3D panel marine hydrodynamic software Hydrostar for head waves and small ship speed of 5 knots Parunov et al. (2015). Although damage extend is very large ( 3 pairs of water ballast tanks in midship region are assumed to be damaged), the effect of the damage on transfer functions of vertical wave bending moments is not significant. The conclusion is similar as in the study of Downs et al. (2007), namely that there is only slight increase in wave-induced response as a consequence of damage.

Reduced exposure time to environmental conditions after damage should be considered before the ships is taken to a safe location. Prestileo et al. (2013) modelled the damaged ship by the added mass approach with 24 hour of exposure to a sea with a heading of $210^{\circ}$ and a speed of 5 knots. Reduced exposure time is counteracted by the hypothesis that the damage has occurred because of adverse sea conditions, so the sea state envisaged after the accident is not a normal but a severe one. The truncated diagram was used by removing half of the complete scatter diagram characterized by lower wave height, less than $8.5 \mathrm{~m}$, and keeping only the most severe sea states. The aforementioned choice of truncation and exposure time yield results in terms of average extreme VWBMs which are approximately $80 \%$ of the corresponding value for the intact ship in her entire life.

Lee et al. (2012c) applied a computational tool based on a 2D linear method to predict the hydrodynamic loads for a damaged warship. They obtained larger VWBM for damaged condition, compared to the intact ship. The global dynamic wave-induced loads calculated using 2D linear method are also compared to measurements. In head and stern quartering waves, the differences between the computations and measurements of global dynamic wave-induced load response amplitudes are reasonable. In general, however, linear strip theory overestimates measurements for both intact and damaged ship conditions.

Stettler \& Thomas (2012) presented a detailed flooding and structural engineering study to accurately model, simulate and evaluate the progressive flooding, sinking and structural failure of the historic Titanic following her collision with icebergs. Detailed computer flooding analysis models were developed, and novel techniques for dynamic flooding simulation using the commercial software GHSTM were developed and implemented. The resulting loads of these detailed flooding simulations were applied to structural FE models using MAESTROTM for conducting detailed stress and hull failure analyses. The technical documentation of the developed computer models, including sources of information, assumptions, conventions and methods, as well as the technical discussion of simulation results and the lessons learned are applicable to similar marine forensic analyses.

Spanos \& Papanikolaou (2012) investigated the time dependence of survivability/floatability and stability of ROPAX vessels, when sustaining side collision damage in beam irregular waves using a 3D and 6 DoF nonlinear time domain simulation method for ship's motions and flooding, where the flood water mass is time-varying and oscillating in a coupled way with the ship motions. Conducted research confirms that ROPAX ships characteristically capsize fast, when sustaining damage leading to capsizing. A probabilistic analysis of the survival time after collision damage reveals that even for the most generic damage conditions assumed, the survival time in the case of capsizing remains short, which is characteristic of this type of ship design, exposing the typically large undivided deck to flooding in higher waves.

Dankowski \& Krüger (2013) presented a progressive flooding method in still water which is based on the calculated flux between the compartments using a modified Bernoulli equation. Large and partly flooded openings are taken into account as well as optional air compression and flooding through completely filled rooms. The method uses a typical damage opening based on the generated damage cubes by a Monte Carlo simulation to perform a direct progressive flooding assessment for each critical intermediate case. The combination of flooding calculations with a Monte Carlo method extends the classical damage stability calculations to the time domain, which allows a more accurate estimation of the overall safety level of a ship to with- 
stand damage. Furthermore, this method is very useful at the early design stage to identify critical intermediate stages of flooding. For the examples shown, a sudden capsize during the flooding process is observed after the vertical centre of gravity is increased by a specific threshold.

\section{Probabilistic modelling of loads on ships}

The ship design process needs to ensure the integrity of the hull, where wave-induced loads contribute significantly. These loads have to be representative of the environmental and operational conditions encountered through the entire life of the ship, which involves statistical representation of the environment and the consequent loads, as well as computationally efficient methods simulating suitable design conditions. This section is, therefore, structured with the aforementioned thoughts in mind.

\subsection{Probabilistic methods}

Stochastic description of loads is required for fatigue spectral analysis and computation of extreme loads for ship design, as well as for the application of ship structural reliability methods. For longitudinal strength assessment of most ship types, basic load variables considered are VWBMs and SWBMs. Load combination of these two components is also considered, accounting for the fact that their maximum values do not occur simultaneously. SWBMs change from one voyage to another and they also vary slowly within each individual journey because of the use of consumables. However, such variation within a voyage is normally neglected in the load combination studies. Load combination of different components of global wave loads, such as vertical and horizontal bending moments (HBM), and torsional moments, and between global and local wave load components are also important (Mohammed et al., 2012).

In recent years, attention, by and large, focussed on ULCSs, where vibratory loads become important. For these flexible ships operating at high speed, the encounter frequency can overlap with the natural hull girder vibration frequency and cause resonance, denoted as springing. This steady state resonant vibration may arise from the two-node flexural mode or the coupled horizontal and torsional mode, the latter only subject to relatively few investigations. Both springing and, transient vibratory, whipping responses are relevant for the fatigue limit state check. For the ultimate limit state check whipping response is more important since it can significantly increase the extreme rigid body VWBM. In recent years a substantial number of studies dealt with stochastic description of combined rigid body and vibratory responses of ULCSs. There appears to be a tendency to consider these two load components together, rather than to study them separately and to combine them using load combination principles. This is partly due to the ascendance in coupled hydrodynamic and structural analyses, providing results of reasonable/acceptable accuracy, and partly due to the nature of model or full scale measurements. In these cases, rigid body and vibratory loads are explicitly included in the analysis and there is no need to combine them. The contribution of the vibratory response can be assessed either by performing analyses with and without elastic effects or by separating low and high frequency signals.

Mao \& Rychlik (2012) presented a simple approach for the prediction of extreme response, for example, 100-year return stress, using Rice's method combined with Winterstein's transformed Gaussian model for stresses. The method requires description of long term variability of the standard deviation, skewness, kurtosis, and zero upcrossing frequency of ship response. It is assumed that the parameters are functions of encountered significant wave height, heading angle and ship speed. The accuracy of this model is validated using the full scale measurements of a 2800 TEU container ship. The parameters in the Winterstein transformation are given by analytical functions of significant wave height only. The proposed method is also used to estimate extreme responses of a 4400 TEU container ship, for which no measurements are available. Results were similar to the 2800 TEU ship. The presented investigation shows that having accurate wave environment model is extremely important for the reliable estimation of the extreme ship responses.

Lee et al. (2012a) presented an analysis procedure for determining values of wave-induced bending moments, considering the effects due to whipping, suitable for design application. The design bending moments due to whipping of the hull girder were determined by multiplying the design rule bending moments by correction factors for hogging and sagging based on the 
Lloyd's Register's guidance notes (2011). The correction factors for hogging and sagging of a 13000 TEU container ship were predicted by employing time domain nonlinear analysis and an Equivalent Design Sea state (EDS). In the nonlinear hydroelastic analysis, the correction factor for hogging was 1.42 for the ship and is $50 \%$ greater than the standard rule hogging correction factor of 0.94 . The correction factor for sagging was 1.98 and is $60 \%$ greater than the standard rule sagging correction factor of 1.24 . These nonlinear bending moments are significantly greater than the standard IACS- based rule wave bending moments.

The effect of hull girder flexibility on the VWBM for ULCSs was analysed by Andersen \& Juncher Jensen (2012). A nonlinear time domain strip theory is used for the hydrodynamic analysis, whilst slamming forces are determined by a standard momentum formulation. The hull flexibility is modelled as a non-prismatic Timoshenko beam. The statistical analysis is carried out using the First Order Reliability Method (FORM) supplemented with Monte Carlo simulations. Strip theory calculations are compared to model tests in regular waves of different wave lengths, and good agreement is obtained for the longest of the waves. For the shorter waves the agreement is less satisfactory. The discrepancy in the amplitudes of the bending moment is explained by an underestimation of the effect of momentum slamming in the strip theory applied.

Ogawa et al. (2012) examined the relationship between the occurrence probability of a slamming induced vibration and sea state. These relationships were investigated based on the full scale measurement data of two large container ships, which operate on the same sea route, but at different periods. The effect of the variability of sea state on the occurrence probability of whipping-induced stress on the hull was examined using the computation of long term prediction and wave hindcast data. The probability of occurrence for high stress is different between the two container ships owing to the difference of period for full scale measurement, though these container ships navigated the same sea route. The stresses are, in general, same for the same wave condition. The difference of probability of wave height has much effect on the whipping and the magnitude of induced stress. It is also clarified that most of slamming induced vibration occurs in head or bow seas. The probability of occurrence for both container ships is consistent with the actual sea state. It is also found that the probability based on the IACS Recommendation 34 (IACS, 2001) is quite larger than the probability in the real sea state. It is noted that the operational effect is not negligible for the evaluation of the occurrence probability of slamming.

Andersen \& Juncher Jensen (2014) analysed full scale measurements of the wave-induced amidships VBM of a 9400 TEU container ship. The focus was on assessing the effect of the hydroelastic high frequency vibration on the extreme hogging VWBM. In the extreme case, they noted that the high frequency vibrations were of the same magnitude as the wave frequency, i.e. so called rigid body, response. It was also noted that even though the ship is sailing in bow quartering seas, only the 2-node vertical vibration mode is apparently excited. The probability of exceedance, for a three hour period, of the amidship VBM in sagging and hogging is shown in Fig. 9, illustrating the influence of hydroelasticity. Following the extreme event analysis and verification, three hours of strain measurements are used for establishing a Gumbel distribution for the extreme value prediction. Extreme value predictions using the measured results indicate that the probability of exceeding the rule design hogging VWBM by $50 \%$ could be of the order of $1.3 \%$ during three hours of operation in a sea state with significant wave height around $8 \mathrm{~m}$.

Teixeira et al. (2013) assessed the probabilistic characteristics of the load combination factors for global still water and wave-induced VBMs of double-hull tankers. The calculations are performed based on loading manuals of oil tankers representative of the range of application of the IACS Common Structural Rules (CSR) design rules. Different load combination methods are used, including an analytical method that provides the combined characteristic value of still water and wave-induced bending moments based on the Poisson assumption for upcrossing events and using the first order reliability method in combination with the point-crossing method. The mean value and the standard deviation of SWBM in one random voyage are defined as $70 \%$ and $20 \%$ of the maximum value in the loading manual, respectively. These values are used for statistical description of sagging bending moments in full load conditions and hogging bending moments for the ship in ballast. For the wave-induced loads, a Weibull distribution was adopted to describe the values of VWBM at a random point in time. A simplified approach for the Weibull model was adopted, in which the shape parameter was assumed as 1.0 and the scale parameter was defined from the IACS rule minimum value defined for design purposes. It was shown that the average load combination factors of the tankers decrease rapidly when the design 
period increases from 1 to 10 years and then tend to stabilize. It was also shown that the mean voyage duration demonstrates a strong influence on the load combination factors. From this analysis, average of load combination factors of 0.84 and 0.81 were obtained, respectively, for large (LTK) and small (STK) tankers in full load and for a reference time period of 20 years. In the ballast load condition, the average of load combination factors reduced to 0.80 and 0.76 for large (LTK) and small (STK) tankers, respectively, which differ on the probabilistic models of the voyage duration and time in port. These factors multiply the most probable VWBM to be added to the most probable SWBM in order to get the most probable combined bending moment.

Mohammed et al. (2012) presented a cross-spectral stochastic analysis methodology for the determination of the combination of global wave-induced dynamic loads. The methodology considers the use of bivariate probability density functions, for the cross-spectral probabilistic approach, or the co-variances of two random variables with their associated derivatives, for the cross-spectral Hamilton's method, and assumes only wave frequency hydrodynamic actions under steady forward speed conditions. A 3D source distribution based on Green's function was applied on the panel model to predict the ship motions and rigid body dynamic wave loads. The design extreme values of global wave-induced load components and their combinations for a container ship progressing in irregular seaways are predicted using these two cross-spectral methods together with the short and long term statistical formulations. It is shown that, in general, both cross-spectral analysis methods can be employed to assess the effects of loads in ship design and reliability analyses. However, the cross-spectral Hamilton's method predicts slightly higher load combinations than the cross-spectral probabilistic approach.

\subsection{Equivalent design waves}

The concept of design waves is used in direct calculations to reduce the number of load cases to be checked for yielding, buckling, ultimate strength or fatigue. A design wave is an equivalent wave or wave group representing the long term response of the dominant load parameter under consideration. Hence, the accuracy of the methodology highly depends on how the design parameters have been chosen. Several types of EDWs exist. General practice in industry is to choose a regular wave for the EDW, the main advantage being its simplicity. The EDW is then defined by the following parameters: frequency, heading, amplitude and phase. However, use of irregular design waves is on the increase, as they are a more accurate representation of irregular sea states. Response Conditioned Wave (RCW) or Most Likely Response Wave (MLRW) are examples of irregular design waves. The notion is to include both the wave spectrum and the ship response in the definition of the Design Wave. de Hautecloque et al. (2012) compared the stress response of a FSRU computed with a long term spectral approach and with a set of equivalent design waves. The approach for selecting the heading of the applied EDW has been shown to have a significant impact on the accuracy of this method, especially when a regular design wave is used to represent short-crested sea states. The irregular design wave, that may include the directional spreading, produces more robust results at the expense of negligible additional computation costs. It is shown that using at least 10 EDWs, the accuracy (standard deviation of the ratio between the EDW stress and the long term stress) is of the order of $6 \%$.

Sarala et al. (2011) proposed a method to derive an EDW from a response based analysis (RBA) to represent extreme loads on a weather-vaning FPSO. In this approach the combined effects of wind, wind-sea, current and swell are considered. RBA is based on three-hourly hindcast metocean data and uses results of heading analysis directly. EDWs are then derived based on the spectral characteristics of each response. This approach is compared with RAO based approach as generally applied in the industry, where the regular design wave is defined only from the RAO characteristics. Six different EDWs are derived based on RBA and RAO methods. It is concluded that the RBA approach provides more realistic responses compared to the RAO based method. Deriving equivalent design waves using only the RAO characteristics is found to give some non-conservative and unrealistic EDWs in some cases

The EDWs are often used to reduce the duration of the simulations to allow for computation of nonlinear loads with more accurate but time consuming software; that is to say, a three hour sea state or even 25 years' life time may be reduced to only a few waves. Derbanne et al. (2012a) used EDWs to compute the 25 years' extreme bending moment of an ULCS including the whipping response. The hydroelastic model couples a 3D hydrodynamic potential solver 
with 3D FE structural dynamics. Slamming loads are computed using a 2D generalized Wagner approach. Results using several type of EDWs are compared with a 53 hour long simulation of a Design Sea State. Regular and irregular design waves are both providing a good estimation of the nonlinear bending moment. However, the method based on an irregular sea state with increased wave height is found to be more accurate, by comparisons with a very long simulation in the design sea state. The significant wave height of the design sea state is increased in order to artificially increase the upcrossing rate of the design value and to save on computation time.

Johannessen \& Hagen (2012) were concerned with the accuracy of the estimation of wave induced design responses from model tests in irregular sea states. They showed that for a highly nonlinear response, such as wave-in-deck loading, it is difficult to have an accurate estimation of the extreme response. By increasing the sea state significant amplitude, it is possible to observe the relevant response more frequently, i.e. increased upcrossing rate; hence, reduce the number of model tests.

Juncher Jensen (2011) combined properties of the FORM analysis and Monte Carlo simulations to better predict the upcrossing rate of a given response. With Monte Carlo simulations the necessary length of the time domain simulations for very low upcrossing rates might be prohibitively long. Using a property of the FORM reliability index, assumed to be valid in the Monte Carlo simulations, makes it possible to increase the upcrossing rates and, thus, reduce the necessary length of the time domain simulations by applying a larger load spectrum than relevant from a design point of view by increasing the significant wave height. The mean upcrossing rate thus obtained can then afterwards be scaled down to its actual value. In this paper the usefulness of this approach is investigated, considering problems related to wave loads on marine structures. Here the load scale parameter is conveniently taken as the square of the significant wave height.

Kim et al. (2012a) estimated the long term midships VWBM and impact-induced bending of two hull forms, both experimentally and numerically. The experimental approach utilizes the lifetime maximum loads analysis based on the Weibull analysis technique. The numerical methods include a series of analyses based on Design Loads Generator (DLG), a tool that can construct an ensemble of short input wave time series, the extreme responses of which follow the theoretical extreme value distribution of a Gaussian random variable for a given exposure time. The exposure time associated with the distribution becomes a good measure by which the associated non-Gaussian responses can be bound. Based on this strategy, the design whipping response has been estimated. To show the responses of comparable exposure time, limited Monte Carlo simulations of combined wave and whipping bending moments are conducted numerically. The DLG approach has the potential to supplement or replace a typical lifetime load analysis based on the combination of operational cells.

Oberhagemann et al. (2012) discussed ways to embed time domain field methods in extreme value predictions, which require appropriate hydrodynamic codes capable of modelling all relevant nonlinearities. Approaches are suggested that appear to give most reliable results. They rely on Monte Carlo simulations, a reduction of parameter variations and extrapolation of exceedance rates over significant wave height. The computational effort is large, yet it can be handled with modern computer clusters. Further studies are recommended to gain more experience on the applicability of the extrapolation over the significant wave height, as well as the uncertainty related to this extrapolation. Finally, the discretization errors are a critical issue, which must be considered in combination

de Hautecloque et al. (2013) evaluated the extreme nonlinear VBM, without whipping, for 70 ships (9 containerships, 19 tankers and 29 bulk carriers) using EDWs or long simulations in short-crested or long-crested sea states. Simulations are carried out in time domain, using linear potential theory with nonlinear hydrostatic and Froude-Krylov forces. It is found that the regular design wave fails to predict the nonlinear hogging bending moment correctly (mean error $-19 \%$, standard deviation 17\%). The irregular design wave, even without spreading, gives a good estimate both in hogging and sagging (standard deviation of the error less than 5\%).

Instead of defining the design wave from the linear response, Clauss et al. (2013) proposed a method that combines a nonlinear strip theory solver with an optimization algorithm to find a critical wave sequence corresponding to a predefined maximum response for the VBM, usually based on a rules value. The obtained shape of the critical wave sequences has been reproduced in the wave tank and model tests have been performed to validate the optimization results. 
Seng \& Juncher Jensen (2012) presented a study of slamming events in conditional waves, under the assumption of rigid body motions. Based on a time domain nonlinear strip theory, most probable conditional waves are generated to induce given short term extreme responses in sagging and hogging VBM at amidships on two container ships. The results of the strip theory are then compared against the results of free surface N-S/VOF CFD simulations, using OpenFOAM, under the same wave conditions. When bow flare slamming occurs the strip theory overpredicts the slamming momentum resulting in higher VBM compared to CFD results. The peak value of the VBM, however, occurs at approximately the same time, implying that the more accurate CFD results can be used to correct the momentum formulation in strip theory through a correction coefficient.

For the structural analysis, EDWs are used to define the few load cases for which structural checks, e.g. yielding and buckling, will be performed. It is important to check that these few load cases are representative of the whole life of the ship. Derbanne et al. (2013a) studied stress response of four different types of structures, comprising conventional ships and unconventional floating structures, using various design waves. Computations are carried out using a 3D linear hydrodynamic solver coupled with a 3D FE solver. It has been shown that with a limited number of design waves, between 5 and 10, the difference between long term linear stress response and EDW stress response is of the order of $10 \%$, for a large range of floating structures. The selection of the governing parameters is, however, the key point of the EDW method. If for a beamlike shape the usual design load parameters, such as VBM, HBM, vertical acceleration at FP and roll angle, are giving good results, it is important for other type of floating structures to define carefully the relevant governing parameters. For unconventional designs, when it is impossible to assume a relationship between a load parameter and the stress response, it is even sometimes necessary to define the EDWs from the linear stress response. In general, the irregular EDWs are performing better than the regular ones, because they are more consistent with the definition of the irregular sea states they are representing. They suffer, however, from a slightly higher bias in the response (underestimate), which can be corrected with a safety factor. On a case by case basis, this approach can be used to define a group of 5 to 10 design waves that represent the worst load cases for a given floating structure. For the development of rule load cases, this approach has to be applied to a large number of ships of different types, different size and different loading conditions. It has to be checked that the same set of governing load parameters can be used for all these ships to define the worst load cases.

\subsection{Design load cases and ultimate strength}

Various design loads used in the strength assessment of ship structures have been introduced by classification societies. Most of these design loads have been determined as standard loads. Hence, the relationship between design loads and sea states actually encountered by ships appear to be weak. Members of IACS developed some common unified requirements and recommendations. The most significant examples are the recommendation $n^{\circ} 34$ (IACS, 2001) defining the wave statistics to be used for the design load computations and the unified requirement S11 (IACS, 2010) providing formulations for VBMs and shear forces.

However, when design loads are computed through direct computations, using the assumptions of recommendation $n^{\circ} 34$, some important differences are found, compared to S11. Derbanne et al. (2013b) compared the nonlinearity of the design VBM (hogging/sagging asymmetry) predicted by the $\mathrm{S} 11$ formulations and computed through a nonlinear seakeeping analysis using design waves and design sea state for 56 different ships. Some important differences were found, especially for containerships, and new formulations are proposed. Work is currently ongoing in IACS to modify the S11 formulations in order to close the gap between direct computation results and rule formulations, and to keep the consistency with the assumptions given in recommendation $\mathrm{n}^{\circ} 34$.

The design loads are then combined to create some load cases, for which structural checks, e.g. yielding, buckling and fatigue, are to be carried out. These load cases are based on the concept of the aforementioned EDWs. Zhu \& Shigemi $(2003,2007)$ developed practical methods for setting design loads. A series of calculations were performed using the strip theory approach for a total of 27 tankers and 22 bulk carriers. 38 wave-induced load components such as ship motions, accelerations, sectional forces and moments and hydrodynamic pressure were considered in this analysis. Dominant loads were identified and regular design waves were used to de- 
fine each load case, using wave encounter angle, wave period and wave amplitude. Four prominent cases are defined as: (i) VBM (head sea), (ii) VBM (following sea), (iii) Roll (beam sea) and (iv) Hydrodynamic pressure at waterline (beam sea).

Harmonized structural rules are developed by IACS wherein common structural rules of bulk carriers and tankers are combined (IACS 2006a, 2006b, 2013). Loads for strength and fatigue assessment are based on recommendation 34 assumptions (IACS, 2001), namely

- North Atlantic scatter diagram,

- Sea states described by a Pierson-Moskowitz spectrum,

- $\cos ^{2}$ angular spreading for wave energy and

- equal heading probability.

The rule load cases for extreme loads and fatigue loads have been defined based on the EDW concept. For extreme loads, a design life of 25 years and a ship speed of 5 knots are considered. Seven EDWs have been defined to generate the dynamic load cases for structural assessment and are shown in Table 1.

For fatigue assessment a ship speed of $75 \%$ of the design speed is considered. The stress history is approximated by a two-parameter Weibull distribution. These parameters are scaling factor and shape parameter. A probability level of $10^{-2}$ is selected for determination of the scaling factor, and the shape factor is taken equal to 1.0. In previous rules, for double-hull oil tankers and bulk carriers, the reference probability level was $10^{-4}$ (IACS 2006a, 2006b). The advantages of the $10^{-2}$ probability level have been explained by Derbanne et al. (2011) as follows: 'the most important contribution to fatigue damage is due to the stress ranges corresponding to a probability approximately $10^{-2}$; if the design stress is directly defined at $10^{-2}$, the influence of the shape factor on the total damage is nearly cancelled; hence, it can then be taken as a constant. Moreover it has been shown that the EDW method can be used to compute the $10^{-2}$ stress.

Five EDWs have been defined to generate the dynamic load cases for structural assessment: HSM, FSM, BSR, BSP and OST, as defined above. The load cases HSA and OSA, defined above, are not considered for fatigue assessment since they are redundant with load case HSM. The approach for combining other responses or subjected loads with the maximized load under the EDW is obtained by the load combination factors (LCFs). The LCFs have been derived through direct analysis for a significant number of oil tankers and bulk carriers covering ballast, full load and intermediate loading conditions.

The hull girder strength is the most critical failure mode for the hull structure. As ships' length becomes longer, this strength is even more important. Hence, the hull girder ultimate strength check is required for ships with length of more than $150 \mathrm{~m}$. The vertical hull girder ultimate bending capacity is to be checked for hogging and sagging conditions, for the following design load scenarios:

- For bulk carriers: design load scenario A, for seagoing, harbour/sheltered water and flooded conditions.

- For oil tankers: design load scenario A, for seagoing and harbour/sheltered water conditions, and design load scenario $\mathrm{B}$, for the operational seagoing homogeneous full load condition.

Partial safety factors to account for material, geometric and strength prediction uncertainties are defined for the aforementioned load scenarios.

\subsection{Weather routing and operational guidance}

Most research with the aim of assisting the master in weather routing is focused on logistic optimization by formulating the problem of minimum time or minimum fuel consumption with constraints. Some efforts are aimed at weather routing taking into account seakeeping behaviour, loads and related measures and the consequences of weather routing on these parameters. These form the subjects reviewed in this sub-section.

The research on weather routing can be divided into four types: (i) research with respect to a suitable and efficient optimization scheme, (ii) simplification of complex hazards in a seaway intended for on-line onboard operational guidance, (iii) monitoring of relevant measures onboard and (iv) a combination of the former three types to form an applicable onboard decision support taking into account the seakeeping behaviour and loads on a vessel.

The current state of the art in weather routing or operational guidance has not changed since the last report of this committee (ISSC, 2012b). The seakeeping characteristics of a vessel are 
considered through the RAOs. In combination with a standard wave spectrum and integrated sea state parameters, such as significant wave height and average zero-crossing period, the linear spectral analysis predicts the response of the vessel for all risk control options. A review of the current state of the art is published by Papanikolaou et al. (2014), where the importance of including uncertainties in the analysis and the consequential need for an efficient probabilistic method are emphasized. A simulation result is presented by Delitala et al. (2010). A route optimization software was applied to the output of a limited area weather model and to a wave model. They concluded that weather routing would improve ship performance and would support ship masters virtually.

A number of papers are devoted to the development of optimum path finding systems with the use of existing environmental data and mathematical models for ship response. Voluntary or involuntary speed reduction is taken into account in some of them; however, little progress can be found with regard to the load estimation. Lee et al. (2011) propose a multi-resolution planning method, which appears to be suitable for including wave effects as well. Panigrahi et al. (2012) advocates the use of Dijkstra's algorithm for a better handling of the voluntary speed loss in the optimization procedure. By applying a Bézier curve for the description of a ship's route, Ishii et al. (2010) demonstrate that only a few variables are needed to generate an optimum route. Though the model has only been tested to minimize transit time, it is possible to include ship responses as well. Lin et al. (2013) proposes a 3D modified isochrome method to be applied for weather routing which is able to address safety concerns. Parametric rolling is taken into account as a risk by Maki et al. (2011), where the real-coded genetic algorithm technique is applied to globally search for the optimum route taking into account of the risk of parametric rolling as one of its objective functions. They concluded that it is easy to obtain a route that reduces parametric rolling probability using the proposed method.

More detailed investigations were carried out with reference to the load/response estimation as part of the routing simulation. A decision-analytic approach is introduced by Nichols et al. (2014), illustrating this approach using a detailed example of a ship in transit, where the goal is to minimize both transit time and probability of structural failure. Assuming that the structural integrity of the ship is governed by the aluminium stringers used in certain naval vessels for hull support, a simple model was used for predicting future damage states and estimating the probability of component failure by means of the spectral approach together with slamming impact force. The main conclusion is that a variable speed strategy compared to a fixed speed strategy is superior and the approach can be made adaptive to incorporate new information such as updated weather predictions. The authors, having run optimal and fixed speed strategies over a number of sea states, obtained expected gain in performance as shown in Fig. 10. It is interesting to note that the more time is allowed for the trip the more flexibility there is to plot a course around challenging sea conditions. Decò \& Frangopol (2013) set up a risk framework incorporating strength elements. In this work the midship section capacity is modelled using FE method including corrosion as a function of time and annual corrosion rate. The load effects are evaluated using strip theory. The dynamic VBMs are accounted for by general approximations based on $\mathrm{L}_{\mathrm{PP}}$ and ships breadth. The framework is applied to the Joint High-Speed Sealift travelling between two points through a selected stormy sea weather map. In the case study the direct risk and reliability are precalculated such that the optimization process takes about 30 minutes. Three representative solutions with different normalized direct risk show that in relatively calm weather the optimum is the direct route, but in harsher weather the optimum route avoids the storm. Mao et al. (2010) presented a simplified fatigue model which can be used in ship routing. The simplified model is based on linear stress transfer functions, while whipping is considered by introducing a modified mean stress upcrossing frequency $\left(\mathrm{f}_{\mathrm{z}}\right)$ based on the wave encounter frequency. Measurements on board a 2800 TEU container vessel showed that $\mathrm{f}_{\mathrm{z}}$ is about $50 \%$ underestimated when using a linear approach. The modification of $f_{z}$ is aimed at reducing the underestimation but accepting that the variability of the encounter frequency is transferred to the modified $\mathrm{f}_{\mathrm{z}}$. The comparison between the empirical distribution of the observed rainfall damage and the cumulated distribution of fatigue damage using the simplified method shows good agreement. The error of the accumulated damage based on the simplified method compared to the measurements is of the order of $10-20 \%$. Mao et al. (2012) applied this procedure to two case studies, a 2800 TEU container vessel and a 4400 TEU container vessel. For most of the voyages, the highest fatigue damage can be decreased by $50 \%$ in the most optimal case. 
Song et al. (2013) present an example of operational guidance to help the crew avoid dangerous situations by precalculations of roll motions using the IRF approach. The results are displayed in occurrence maps of parametric roll as a function of speed and heading, for a range of GM values and sea states. The authors address neither how the precalculations are efficiently presented to the crew nor the required quality of the input parameters, such as sea state and loading condition.

\section{Fatigue Loads for ships}

With increases in size of container ships, the contribution of high frequency vibrations to the fatigue strength/damage of the hull girder has been extensively studied in recent years. The peak values of the VBM and the resulting bending stress increase as a result of the high frequency vibrations due to whipping and springing of the ship. This observation is based on analysis of full scale measurements. It is not possible to distinguish springing from whipping in measurements; hence, most publications refer to the high frequency vibration as whipping, since it is usually considered that springing occurs in relatively calm seas. This section mainly deals with the effects of high frequency vibrations on the fatigue strength of ships.

Andersen \& Juncher Jensen (2014) analysed the full scale VWBM measurements of a 9400 TEU container ship in bow quartering seas by using FFT and concluded that the high frequency vibrations caused by impulsive loads are observed to be of the same magnitude as the rigid body response and, thus, act so as to double the total VBM amidships in the extreme case, as discussed more extensively in Section 10.1. Storhaug et al. (2012) showed that this high frequency vibration contributed to increase the dynamic extreme stresses on deck amidships by $22-33 \%$ in hogging and $27-32 \%$ in sagging by analysing the maximum values every half hour. Their conclusions were based on the full scale measurements from two LNG vessels operating in typical worldwide trading patterns for more than 5 years, excluding the Trans-Pacific trade. Based on elastic backbone model test data of two ULCSs, 8600 TEU and 13000 TEU, respectively, in regular and irregular head waves, Zhu \& Moan (2013) showed that the high frequency vibrations increase the sagging VBMs more significantly than the hogging VBMs, however, the hogging peaks are increased more by the high frequency vibrations in relatively short waves because of the behaviours of second and third harmonics.

After fatigue damage was identified on a large ore carrier, attributed to whipping, its effect on longitudinal strength was further examined. Onboard measurements and model tests with flexible models were performed, and it was shown that the contribution of the rigid body response to the total fatigue damage was relatively large (ISSC, 2012b). Storhaug (2012) showed that the high frequency vibration contributes to approximately $26 \%$ of the fatigue damage on a 2800 TEU container ship and $29 \%$ of the fatigue damage on a 4400 TEU container ship, based on measurements in the North Atlantic for a few years. Toyoda et al. (2012) applied the rainflow counting and Miner's rule to the full scale measurement data of two post Panamax container ships taken over a period of 2.5 years and showed that the fatigue damage is increased 3.9 times due to the effect of whipping. It should be noted, however, that the absolute value of fatigue damage is quite low, $10-{ }^{3}$ level, say. Based on measurement campaigns onboard a Panamax and a post Panamax container ships, the effect of high frequency hull girder vibrations on fatigue damage was assessed by Rathje et al. (2012). The contribution of high frequency loads was found to be $35 \%$ of total damage for the Panamax ship, while it was $57 \%$ for the post Panamax ship. They concluded, however, that the screening of damage data bases for Panamax container ships indicated that no significant amount of damage had occurred on similar ships, even for ships operating worldwide for more than 20 years. Andersen \& Juncher Jensen (2013) estimated the fatigue damage applying the spectral analysis to the full scale stress measurements onboard the hull of a large container vessel, 9400 TEU, during several months of operation. They concluded that the spectral analysis show satisfactory agreement with the results from rainflow counting. Fatigue damage was also evaluated using the model test data and numerical simulation, and the effect of high frequency vibration due to whipping and/or springing was discussed. Derbanne et al. (2012b) computed linear long term fatigue damage for 6 container ships based on bending moment RAOs. The conclusion is that for vertical bending damage the hydrostatic restoring effect and the low frequency dynamic amplification effects are cancelling out each other. 
Wang et al. (2013) predicted springing responses of large ships using 3D hydroelastic theory and model experiments. They showed that the structural fatigue damage induced by combined wave loads is much more severe than that induced by rigid body loads when wave period is small. Choi et al. (2013) numerically evaluated the hull-girder responses including the springing effect for an ULCS based on coupling of Vlasov beam theory and time domain 3D Rankine panel method. They concluded that hydroelastic effect is not reflected sufficiently in the numerical simulation for harsh sea conditions when compared with the results of model tests. Koo et al. (2013) carried out the fatigue assessment of an 18000TEU container vessel using a spectral approach and taking into account the springing effect. They concluded that the fatigue damage due to the springing effect has been between $24 \%$ and $64 \%$ of total fatigue damage when carrying out a fatigue damage analysis; however, fatigue damage considering the springing effects of case studies with measured data is much lower than results from the analysis.

Usually the rainflow counting method of stress cycles, considering the memory effect during elastic-plastic material behaviour in notches, as well as the Palmgren-Miner rule, are applied to predict the fatigue damage under variable amplitude loading, yielding the so called life curve compared to the S-N curve. In the Palmgren-Miner rule, the damage due to small stress cycles below the knee point of the S-N curve, i.e. fatigue limit, is usually considered assuming a modified slope exponent of the S-N curve beyond the fatigue limit, i.e. the so called Haibach's correction. Another parameter affecting fatigue life prediction is the bandwidth of the stress spectrum, which becomes wider in cases when the load process contains several frequencies. A rainflow correction factor was introduced to consider bandwidth effects on rainflow counting and damage summation for different spectra and bandwidth (Fricke \& Paetzold, 2013).

Fricke \& Paetzold (2012) performed fatigue tests using simplified stress histories with superimposed stress cycles of different frequencies and concluded that almost the whole of the fatigue damage is caused by the stress cycles induced by wave-frequency (rigid body load) which is enlarged by whipping, whereas the additional small stress cycles can be neglected. Fricke \& Paetzold (2013) also performed fatigue tests of a welded detail with variable amplitude loading to investigate the effect of whipping stresses on fatigue damage in ships and the suitability of the Palmgren-Miner rule for fatigue life assessment. Stress histories comprising of two sinusoidal functions and a stress history obtained from onboard measurements were applied. The traditional approach with rainflow counting and linear damage accumulation based on the PalmgrenMiner rule is found to be suitable for ship-typical stress histories with low-frequency stress cycles due to waves and high-frequency stress cycles due to whipping. Most of the fatigue damage is caused by the low frequency stress cycles, enlarged by whipping, as long as the whipping stress amplitudes are smaller than the wave-induced rigid body stress amplitudes. The contribution of the additional small stress cycles due to whipping is rather small. Osawa et al. (2013) developed new simple fatigue testing machines which can carry out fast and low cost fatigue tests of welded joints subject to high frequency vibration superimposed on to wave loadings. Fatigue tests of out-of-plane gusset welded joints subject to springing and whipping, superimposed on to wave loadings were carried out. They also showed that the enlargement effect of the total stress range has a dominant influence on the fatigue strength under the conditions chosen. However, they concluded that it is unclear whether similar results can be found for the cases under various wave loadings with high frequency vibration.

On the other hand, Gotoh et al. (2012) performed fatigue tests of centre-cracked tensile specimens with simplified stress cycles containing high cycle stresses with/without damping, which is attained by the transient stresses being 5 times as high as the cyclic stress. Tests are also conducted with the regular low cycle stresses representing the envelope of the simplified stress cycles. Numerical simulation of fatigue crack propagation based on an advanced fracture mechanics approach using the RPG (Re-tensile Plastic zone Generating) load criterion for fatigue crack propagation is conducted and an extracting procedure for the effective loading sequence during random loading is proposed with the use of RPG load criterion. The test results were compared with the traditional S-N approach using the more advanced RPG load criterion which was superior to the ordinary cycle counting method such as rainflow method, because the load sequence effects on fatigue life can be taken into account. It was found that the fatigue life was underestimated in some cases, which were mainly attributed to irregular stress cycles (history) or deceleration/delay in fatigue crack growth when small stress range cycles followed a large stress range cycle, which is usual in the case of whipping. Furthermore, it was also confirmed from the 
experimental results that the traditional fatigue life evaluation method based on the S-N curves approach with rain flow counting is inappropriate under superposed loading conditions. Although the enlargement effect of stress amplitude by high frequency component is a very simple way for the consideration of whipping stresses in fatigue analyses, it is pointed out that uncertainties still exist regarding the real fatigue damage under typical stress histories containing wave loads and high frequency vibrations.

It is accepted that the stress amplitude of a ship's hull girder increases due to whipping and springing vibrations, and that fatigue damage also increases as a consequence of the enlargement effect due to high frequency vibration components. This fact is confirmed through comparisons with fatigue test data using test pieces in several cases. Nevertheless, it is also an undeniable fact that damages attributed to such hull girder vibrations were rarely reported for wellmaintained, properly operated ships. One reason for such occurrence may be attributed to the nature of the Palmgren-Miner rule. Fukasawa (2012) showed, from numerical simulation results, that the number of stress cycles increases also in the zero-crossing counting case, but the increase rate is lower than that corresponding to the local peak counting case. Derbanne et al. (2011) and Fukasawa \& Mukai (2013) showed in their numerical simulations that the dominant stress range to the fatigue damage is that of relatively small amplitude whose occurrence probability is approximately $10^{-2}$ according to Miner's rule, and a certain stress range has a significant effect on the fatigue damage.

Another reason may be attributed to the ship handling and the seaway conditions encountered. It is quite usual nowadays to operate a container ship at a speed less than 20 knots to save fuel and the ship can avoid rough weather due to advanced weather forecasting. The operational effect is not negligible for the evaluation of the occurrence probability of slamming. Ogawa et al. (2012) investigated the effect of different sea states on the occurrence probability of whipping-induced stress. Their work was based on full scale measurements of container ships, and the long term predictions were conducted based on the measured stresses and wave hindcast data. They showed that the difference of probability in wave height has significant or large effect on whipping and the consequent magnitude of induced stress. They also noted that most of slamming induced vibration occurs in head or bow seas. Storhaug (2012) measured strains and wind speed and direction on 2 container vessels (2800 TEU and 4400 TEU) and showed that fatigue damage is largest in head seas for the larger vessel and bow quartering seas for the smaller vessel, and that the relative vibration related damage decreases from head to stern seas. Based on fatigue tests and fatigue crack growth simulation, Sumi et al. (2013) stated that shipping routes and ship operation, including influence of ship heading and speed, must be taken into consideration for the proper estimation of probabilities. Prasetyo et al. (2012) conducted fatigue crack propagation based assessment of a surface crack in a weld of a longitudinal stiffener using JWA (Japan Weather Association) hindcast data for the north Pacific Ocean region. Load history is generated based on third generation storm model, which can take into account fluctuations in storm duration. It was shown that the fatigue damage ratio of second generation storm model, which can only take into account the relation between wave height and wave period of the short term sea state, to third generation storm model is almost equal to the ratio of calculated crack propagation life in each storm. The proper choice of wave environment and seagoing condition is important in evaluating the effects of whipping and springing.

\section{Uncertainty analysis}

This section reviews only uncertainties related to wave-induced loads and loading conditions.

\subsection{Load uncertainties}

The subject of modelling and implementing uncertainties in maritime technology related areas is vast and different approaches are used, mostly depending on the aim of the analyses. Thus, Papanikolau et al. (2014) presented recent advances in the treatment of uncertainties related to two engineering applications, namely development of reliability-based code formats and development of modern decision support systems (DSS) as guidance to a ship's master. Whilst both applications of uncertainty modelling share some common issues, there are also obvious differences. For example, in the former application, there is a basic uncertainty of design scenario for ship operation, requiring appropriate selection of parameters and calculation methods. Accordingly, the general practice has been to adopt the North Atlantic wave climate as a reference con- 
dition because of its severity. The counter argument is that real operational scenarios should be used in probabilistically based design. Even if a ship route, other than the North Atlantic one, is known at the design stage, ship owners argue that the ship could be sold during her lifetime and possibly be used in the North Atlantic trading routes (Guedes Soares 1996).

Such uncertainty is not important for the second application, i.e. the development of DSS, where other uncertainties dominate. One of the important uncertainties in DSS relates to the definition of threshold or limiting values of seakeeping parameters that are used to predict the sustainable ship speed and heading in certain sea conditions. General operability limiting criteria, such as RMS of vertical acceleration at FP, probability of slamming, probability of green water and probability of propeller emergence are usually defined as fixed values depending on ship size and type and then used for design purposes (Moan et al., 2006). However, for probabilistically based DSS more refined seakeeping criteria need to be defined, preferably as random variables, and then included in the limit state function for evaluation of different seakeeping hazards (Papanikolau et al. 2014).

Accuracy of observations or measurements relating to parameters of sea state in which a ship is sailing is one of the dominating uncertainties in applications of DSS. Nielsen et al (2013) produced sea state estimates by three different means: the wave buoy analogy whereby one is relying on onboard response measurements, a wave radar system, and an onboard system providing the instantaneous wave height. The presented results show that for the given data, recorded on five different days of continuous operation, the agreement between the estimated means is reasonable.

The principal global loads causing longitudinal hull bending of ship may be summarized as follows (Bai, 2003): (i) still-water bending moments, (ii) rigid body (wave frequency or quasistatic) wave bending moments and (iii) vibratory bending moments caused by whipping and springing. The still water bending moments result from the longitudinal distribution of the cargo onboard and other controllable factors which are changing for each departure condition. Although still water loads are deterministic in nature, during a ship's lifetime they fluctuate; hence, they can, and should, be considered as stochastic process.

Rigid body VWBMs are obtained from standard seakeeping analysis. Primary uncertainties sources in numerical seakeeping analysis, according to Kim \& Hermansky (2014), are :

- different mathematical modelling of (initial) boundary value problem,

- different numerical modelling of the assumed mathematical model,

- non-converged or inaccurate hull geometry modelling,

- insufficient or incorrect knowledge regarding mass distribution, and

- human, i.e., user, error.

In addition, uncertainties at different stages of spectral analysis, which according to Hirdaris et al. (2014) need to be also considered, are:

- type of wave spectra,

- the choice of wave scatter diagram,

- method for prediction of long term extreme values and

- the operation of the ship (human actions/factors).

As the aforementioned uncertainties relate to the linear hydrodynamic tools only, computational uncertainty of nonlinear effects in wave loads, especially different sagging and hogging bending moments, should be added to the total uncertainty.

Transient elastic vibrations of the ship hull girder due to whipping represent important contributions to the VBM of large slender ships, especially ULCSs. Whipping vibrations increase both the extreme and fatigue load effects occurring in ships. VBMs induced by the combined effect of wave bending and whipping can double the magnitude of the former, as previously discussed in Section 11. Thus, considerable efforts have been spent on predicting loads associated with slamming and consequent structural response. Drummen \& Holtmann (2014) presented results of benchmark study on uncertainties in slamming and whipping undertaken in 2012 by ISSC. The ship used in this benchmark study was $173 \mathrm{~m}$ long RO/RO ferry. Surprisingly, they noted large discrepancies, even in the estimate of the two lowest natural frequencies of the global flexural vibration modes. However, it is interesting to note that overall results, especially for 3-node vibration, are in better agreement to the experiment for wet than for $d r y$ modes. When participants applied different realistic, analytical, pulses to their model, significant differences in fatigue loading of up to a factor of five were observed. Furthermore, the computations consider- 
ing the response to an impulse induced by a regular head wave showed significant differences between the experiment, the different participants, and applied methods. The computation of slamming loads was performed i) using 2D slamming sections and BEM, ii) hybrid approach for slamming pressure computation, where initially vessel motions were computed using a Rankine panel method and imposing these motions on a RANS model using ANSYS CFX, iii) as per ii) but the motions were calculated using RANS solver StarCCM+, iv) fully coupled FSI computation based on RANS solver Comet for fluid dynamics and Timoshenko beam structural representation. The resulting fatigue damage compared to the experimentally measured loads was in the range of $0.42 \%$ to $507 \%$, where experimental fatigue damage reads $100 \%$. The authors concluded that the benchmark emphasized the need to validate and gain personal experience with dedicated tools.

Ćorak et al. (2013) carried out a parametric study of the influence of container ship route and operational and environmental restrictions on the long term distribution of combined, rigid-body and vibratory bending moments. The problem is formulated in the frequency domain using standard engineering tools for load computation: a 3D seakeeping code for the rigid body response and a beam FE model for transient vibratory response. The von Karman approach, with correction for pile-up effect, is employed for bow flare slamming load assessment. As expected, the IACS rule VWBM is largely exceeded by the combined VBM for the North Atlantic sea environment. The choice of alternative shipping routes can significantly reduce the long-term extreme combined VBM. Thus, combined VBMs could be reduced by $6 \%$ in the case of the North Pacific and by $26 \%$ in the case of the Far East-North European shipping route. Voluntary speed reduction has a considerable influence on the total VBM. By employing the speed profile reduction from ship operability analysis, the combined VBM for the North Atlantic route could be reduced by $21 \%$ for a constant ship speed of 15 knots.

Research related to the whipping effects of container ships has intensified after the accident of the container ship MSC Napoli in the English Channel in 2007. Although whipping certainly occurred at the time of the accident, it is still not clear how much it actually contributed to the hull girder failure (Storhaug, 2009). Parunov et al. (2014), in their investigations of the MSC Napoli, proposed the structural reliability approach, which enables identification of the relative importance of various pertinent variables on the structural failure. Sensitivity analysis, which is regular part of the structural reliability study, showed that the most important random variable at the time of the accident appears to be the modelling uncertainty in the bending capacity $(32 \%)$. Such large uncertainty is caused by the uncertainty in the material yield strength and the modelling uncertainty of the progressive collapse analysis method used to assess the hull girder vertical bending capacity. However, the overall importance of all variables related to the uncertainty of the rigid body VWBM (37\%) exceeds the importance of the strength uncertainty. The overall importance of the whipping contribution (24\%) is approximately $2 / 3$ of the importance of the rigid body VWBM.

The importance of uncertainties in design and analysis of large container ships was emphasized once again due to the case of structural failure of the MOL Comfort container ship in the Indian Ocean in June 2013. The report issued by the Maritime Safety Committee (IMO, 2014a) reveals that the estimated total global bending loading at the time of the accident was of the order of $9.4 \mathrm{GNm}$, whilst the estimated bending moment capacity was $14.0 \mathrm{GNm}$. This indicates that the estimated load was approximately $67 \%$ of the hull strength and the conditions for fracture could not be simulated. For this reason, the authors of the report concluded that it would be necessary to consider uncertainties in further verification of both load and strength related calculations.

\subsection{Uncertainties in loading conditions}

The uncertainty of the weight and weight distribution and their consequences have not been the subject of scientific analysis in the usual journals, nor any ISSC committee. Nevertheless, these uncertainties have been identified by industry as a considerable problem, and not only in the context of the recent ship accidents of MOL COMFORT and MSC NAPOLI. According to the world shipping council some customs, namely Ukraine, Poland and India, have investigated container weights. The IMO, in May 2014, approved draft amendments to SOLAS chapter VI requiring mandatory verification of the gross mass of containers (IMO, 2014b), which were approved in November 2014. 
Within the European research project ADOPT (Nielsen \& Michelsen, 2007) the variation of deadloads corresponding to six months' loading conditions from a RORO vessel were analysed. The analysis showed deadloads up to $16 \%$ of the displacement, that is up to approximately 1300 tonnes, with the dominant range from 200 to 900 tonnes. Deadload is the excess between the vessel's calculated deadweight, based on draught readings, and known weights such as cargo, fuel ballast, water etc. The uncertainty of cargo weight and distribution for RORO, ROPax and heavy lift vessels is mentioned in the report of the research project lashing@sea but it is concluded that since the accident rate has not increased, it is not considered a problem for such ship types (Koning, 2009).

Cargo that is heavier than declared is not only a problem for the establishment of maximum allowable loads on ships but also an important factor in the collapse of container stacks, loss of containers at sea, stability failure and transportation on shore. One of the issues noted within the lashing@sea project was that no centralized data base of shipping incidents of cargo loss and damages are kept (Koning 2009). This makes an analysis of the extent and severity of the uncertainty of cargo weight difficult.

Within the investigation of major structural failures of container ships, the uncertainty of cargo load has been evaluated whenever possible. The accident reports of the MSC NAPOLI (MAIB, 2008) stated that this ship often had large deadloads on completion of loading. The deadload on departure from Antwerp, prior to the cracking, was about 1250 tonnes (approximately $1.6 \%$ of displacement). During the removal of containers, the position of 700 containers on deck were compared with the position recorded by the terminal operator. Of these units 53 $(7 \%)$ were in the wrong position or misdeclared. It is generally agreed within the container industry that up to $10 \%$ of containers loaded onto a vessel might not be in their planned positions. Since the two parts of the MOL COMFORT sank, the contribution of the uncertainty of weight and weight distribution cannot be accounted for. The effect of the uncertainty of container cargo weight on the SWBM, based on the hull deflection obtained through draught measurements at the time of departure, is difficult to assess (MAIB, 2008).

A submission to IMO (2012) summarizes some incidents due to, amongst others, misdeclaration of container weights. For example,

- Containership Deneb in Algeciras (CPIAIM, 2012): According to this report, the weight of the cargo used for calculating the stability was 332 tonnes, approximately $9 \%$ of displacement, lower than the calculated weight;

- P\&O Nedlloyd Genoa (MAIB, 2006): Two containers were overweight, but the results show that the remaining containers were close to their declared weights.

Based on information obtained from the world shipping council it is known that the Ukrainian customs, over a two week period in October 2012, weighed all packed containers discharged in Ukrainian ports. $56 \%$ of the containers had an actual weight greater than the weight stated in the manifest based on the shipper's declared weight as provided in the shipping instructions.

Based on current evidence, it is difficult to speculate on the consequences of the uncertainties in loading conditions. In the case of the structural failure of MSC NAPOLI the overloading of the vessel, i.e. the resulting deviation to the SWBM, would have been extremely small by comparison to the potential variability of the wave loading. Therefore, the effect of the discrepancies alone would have been insufficient to cause hull failure. Nevertheless, they would have contributed to the reduction of the safety margin available (MAIB, 2008). Whether this conclusion holds true for other vessels cannot be established due to poor and incomplete evidence.

\section{Conclusions}

On Computational methods for evaluating wave-induced loads: Potential flow solutions continue, due to their efficiency, to be dominant for zero and non-zero forward speed scenarios, albeit with inclusion of a range of nonlinearities. Therefore, further development of 3D fully nonlinear potential flow solvers is still valuable. The focus in these applications is the development of efficient, practically applicable numerical methods and software, capable of coupling with phenomena, such as slamming, sloshing and green water. Use of N-S solvers is increasingly focusing on the seakeeping problem with improved accuracy of predictions. Hybrid solutions for computational efficiency, either within potential flow solvers, namely coupling near and far 
field approaches, or involving the coupling of nonlinear potential flow with N-S solvers in the near field, are amongst the promising developments.

It is interesting to note that the state-of-the-art for either zero or forward speed cases is very similar, in spite of differences in operational and environmental frameworks. Furthermore, the need for measurements to validate computational methods remains paramount for both cases.

On Hydroelasticity: The main progress in hydroelasticity analysis is in the development of BEM based nonlinear FSI methods through coupling with FE models. Coupling between RANS and FE solvers, currently used for slamming related problems, is also being applied to predict the rigid body and springing related loads. The success of the latter will depend on the computational efficiencies made and the improvements in accuracy obtained with reference to potential flow based predictions.

On Slamming: Prediction of slamming loads has recently covered some aspects that were of secondary importance in the past with respect to the determination of the pressure peaks over the impacting bow: water-exit phase, presence of both vertical and horizontal components in the water-entry velocity, oblique or asymmetric impacts, wavy surface are some of them. Hydroelastic effects have been dealt with different approaches ranging from semi-analytical methods to coupled finite element and RANS solvers including 3D fluid-structure interaction and composite materials. Indeed, many of these studies have been oriented toward analysis of containerships since their flexibility poses challenging questions to numerical simulations.

On Green water: The green water problem has been investigated experimentally and numerically, but with very limited success. The issues arise due to the fact that green water is very difficult to measure either under laboratory conditions or in the field, and very difficult to simulate numerically. The problem, due its fast moving, multiphase and highly turbulent nature, is not very amenable to accurate numerical simulation, although 3D models help to better understand and simulate the mechanisms of green water impinging on deck. Green water load computation with various wave headings is still a challenge. Qualitative benchmark studies are required to validate and improve numerical approaches.

On Sloshing: The majority of investigations into sloshing focus on numerical simulations using RANS CFD methods and experimentation, 3D simulations and realistic excitations for both. Analytical investigations can be useful in terms of providing simple formulae useful in design. Recent advances include studies on sloshing with internal suppressing structures and investigation on the coupling between sloshing and ship motions, both numerically and experimentally. It is expected that these studies will continue in order to improve the accuracy of numerical simulations.

On Loads from abnormal waves: Research and early findings raise the some critical questions: should we design future ships for abnormal waves or for the upper tails (essentially beyond $99 \%$ quintiles) of wave statistics and will be these ships economically viable? Here we need to differentiate between merchant and naval ships, as their mission profiles differ significantly. Also, the design problem of offshore structures, which cannot avoid extreme environmental loads, is inherently different from that of ships, for which operational measures and guidance to the master to avoid dangerous environmental loads appear more efficient than an increase of design loads and safety factors.

On Measurements: Recent advances in measurement techniques and fabrication technology have indeed broaden the capability to design experimental setups where scaled models can reproduce the full-scale elastic behavior and provide high quality data for code validation. Thus, much effort focused on the assessment of physical models to be used in FSI tests and their mechanical and structural properties. The analysis of transient phenomena, such as slamming, has benefited from the application of advanced signal analysis techniques. Full scale monitoring of environmental conditions and hull structures has also greatly improved, thus allowing for real ship response investigations at a reasonable cost, as well as assessing our capability for modeling numerically and experimentally. 
On Loads following damage: The motivation for increasing research on damaged ship is the implementation of risk based methods in rule development, where residual strength of ships in different foreseen damage situations is required in addition to that of the intact ship. The use of reliability based methods demands realistic estimates of loads on damaged ship. Thus, the main driving force for further research, is the development and understanding of both deterministic and probabilistic models for load assessment in accidental situations.

On Probabilistic methods: These are, by and large, employed to study short and long term distributions of ship responses. Load combination studies of still water and a range of wave loads are also performed. Although a significant amount of research is dedicated to the designoriented probabilistic methods for prediction of extreme combined wave-induced and vibratory responses, we are of the opinion that practical methods, applicable for conceptual and preliminary ship design, are still lacking.

On Design methods for ships: The focus of investigations has been on developing and verifying equivalent design waves. The selection of the characteristics of such waves, such as increased sea state significant wave height, aids in cutting down the amount of simulations required.

It is expected that the rule based approach and the direct calculation approach should give same answer in terms of the acceptance of the particular ship structural design in the near future.

On Weather routing: Development of optimization schemes and simplified mathematical models for complex hazards, such as fatigue damage form the basis of the research into weather routing. The necessity of embedding an uncertainty analysis into an operational guidance has been identified, but not accounted for in most research. The reliability of the guidance, e.g. identifying and mitigating false predictions, has not been an issue; neither the transmission of information gained to the master, the crew and the ship operators to enable sound decisionmaking under uncertainties.

On Fatigue loads for ships: Crack initiation and propagation behaviours are nonlinear phenomena and there may be some limitations in the application of Miner's rule in terms of accuracy of estimated fatigue damage. Further investigations are necessary to clarify the effect of hull girder vibration upon the fatigue strength of a ship by accounting for stress histories which are effective in crack initiation and propagation. Full scale measurements, model tests and numerical simulations, such as the aforementioned crack propagation analyses, should be combined to clarify the fatigue life characteristics of a ship in waves.

On Uncertainties: The importance of uncertainties can be seen in comparative studies predicting ship motion and structural loads, such as that on a large containership arranged by the 2nd ITTC-ISSC Joint Workshop in 2014. We are of the opinion that systematic uncertainty assessment is one of the main issues requiring resolution for the advancement of marine structural design. Development of sophisticated computational tools should be followed by uncertainty analysis of their predictions, including operational uncertainties as one of the main contributing factors in definition of design loads.

This review indicates two specific trends for fluid-structure interaction problems: (i) a systems approach which should be extended to include coupling between propulsion/control devices, in ships, and mooring/riser systems, in marine structures, to enable a more accurate and complete evaluation of loads; (ii) the pursuit of hybrid solutions for computational efficiency, which should continue and expand towards data fusion, namely solution systems bringing together a range of computational predictions and measurements, and making use of data-rich and datasparse analyses.

ACKNOWLEDGEMENT

Dr. Parunov acknowledges the support of the Croatian Science Foundation under project 8658.

\section{References}


Aarsnes, J.V. 1996. Drop test with ship sections-effect of roll angle. Report 603834.00.01. Norwegian Marine Technology Research Institute, Trondheim.

Abrahamsen, B. \& Faltinsen, O. 2011. The effect of air leakage and heat exchange on the decay of entrapped air pocket slamming oscillations. Physics of Fluids 22, 102107.

Abrahamsen, B. \& Faltinsen, O.M. 2012a. The natural frequency of the pressure oscillations inside a water-wave entrapped air pocket on a rigid wall. Journal of Fluids and Structures 35: 200-212.

Abrahamsen, B. \& Faltinsen, O. 2012b. A numerical model of an air pocket impact during sloshing. Applied Ocean Research 37: 54-71.

Abrahamsen, B. \& Faltinsen, O. 2013. Scaling of entrapped gas pocket slamming events at dissimilar Euler number. Journal of Fluids and Structures 40: 246-254.

Akyildiz, H., Erdem Unal, N. \& Aksoy, H. 2013. An experimental investigation of the effects of the ring baffles on liquid sloshing in a rigid cylindrical tank. Ocean Engineering 59: 190-197.

Andersen, E.A. \& Juncher Jensen, J. 2013. Hull girder fatigue damage estimations of a large container vessel by spectral analysis. In Proc. $12^{\text {th }}$ Int. Symp. on Practical Design of Ships and Other Floating Structures PRADS, Changwon, Korea.

Andersen, I.M.A. \& Juncher Jensen, J. 2012. On the effect of hull girder flexibility on the vertical wave bending moment for ultra large container vessels. In Proc. $31^{\text {st }}$ Int. Conf. on Ocean, Offshore and Arctic Engineering OMAE, Rio de Janeiro, Brazil.

Andersen, I.M.A. \& Juncher Jensen, J. 2014. Measurements in a container ship of wave induced hull girder stresses in excess of design values. Marine Structures 37: 54-85.

Antuono, M., Bouscasse, B., Colagrossi, A. \& Lugni, C. 2012. Two-dimensional modal method for shallow-water sloshing in rectangular basins. Journal of Fluid Mechanics 700: 419-440.

Ariyarathne, K., Chang, K.A. \& Mercier, R. 2012. Green water impact pressure on a three-dimensional model structure. Experimental. Fluids 53:1879-1894.

Bai, Y. 2003. Marine Structural Design. Elsevier.

Barhoumi, M. \& Storhaug, G. 2013. Assessment of whipping and springing on a large container vessel. In Proc. $12^{\text {th }}$ Int. Symp. on Practical Design of Ships and Other Floating Structures PRADS, Changwon, Korea.

Bennett, S. S., Hudson, D. A. \& Temarel, P. 2012a. A comparison of abnormal wave generation techniques for experimental modelling of abnormal wave-vessel interactions. Ocean Engineering 51: 3448.

Bennett, S. S., Hudson, D. A., Temarel, P. \& Price, W. G. 2012b. The influence of abnormal waves on global wave-induced loads, In Proc. $6^{\text {th }}$ Int. Conf. on Hydroelasticity in Marine Technology, Tokyo, Japan.

Bennett, S. S., Hudson, D. A. \& Temarel, P. 2013. The influence of forward speed on ship motions in abnormal waves: experimental measurements and numerical predictions. Journal of Fluids and Structures 39: 154-172.

Bennett, S. S., Hudson, D. A. \& Temarel, P. 2014. Global wave-induced loads in abnormal waves: comparison between experimental results and classification society rules. Journal of Fluids and Structures 49: 498-515.

Bitner-Gregersen, E., Hørte, T., Eide, L. \& Vanem, E. 2014. Impact of climate change and extreme waves on tanker design. SNAME Annual Meeting, Houston, USA.

Brard, R. 1948. Introduction à l'étude théorique du tangage en marche. Bulletin de l'ATMA France 47: 455-479.

Buchner, B. \&. van den Berg, J. 2013. Non-linear wave reflection along the side of ships leading to sreen water on deck. In Proc. $12^{\text {th }}$ Int. Symp. on Practical Design of Ships and Other Floating Structures PRADS, Changwon, Korea.

Burić, Z., Bužančić Primorac, B. \& Parunov, J. 2012. Residual strength of damaged oil tanker in the Adriatic Sea. In Proc. $17^{\text {th }}$ Int. Conf. on Ships and Shipping Research NAV, Naples, Italy.

Carvalho, D.F.\& Rossi, R.R. 2014. Green water loads determination for FPSOs exposed to beam sea conditions. In Proc. $33^{\text {rd }}$ Int. Conf. on Ocean, Offshore and Arctic Engineering OMAE, San Francisco, $U S A$.

Chapchap, A.C., Miao, S.H, Temarel, P. \& Hirdaris, S.E. 2012. Time domain hydroelasticity analysis: the three-dimensional linear radiation problem. In Proc. $6^{\text {th }}$ Int. Conf. on Hydroelasticity in Marine Technology, Tokyo, Japan.

Chen, Z., Zong, Z., Li, H. \& Li, J. 2013a. An investigation into the pressure on solid walls in 2 d sloshing using SPH method. Ocean Engineering 69: 129-141.

Choi, J.H., Jung, B.H. \& Hwang, J.H. 2013. Evaluation of springing-induced fatigue damage for ultralarge container carrier. In Proc. $23^{\text {rd }}$ Int. Offshore and Polar Engineering Conference ISOPE, Alaska, USA. 
Clauss, G.F, Klein, M., Guedes soares, C. \& Fonseca, N. 2013. Response based identification of critical wave scenarios. Journal of Offshore Mechanics and Arctic Engineering 135.

Ćorak, M., Parunov, J. \& Guedes Soares, C. 2013. Long-term prediction of combined wave and whipping bending moments of container ships. Ships and Offshore Structures.

CPIAIM. 2012. Investigation of the capsizing of merchant vessel DENEB at the Port of Algeciras on 11 June 2011. Technical report A-20/2012 Comisión Permanente de Investigación de Accidentes e Incidentes Marítimos.

Dankowski, H. \& Krüger, S. 2013. Progressive Flooding Assessment of the Intermediate Damage Cases as an Extension of a Monte-Carlo based Damage Stability Method. In Proc. $12^{\text {th }}$ Int. Symp. on Practical Design of Ships and Other Floating Structures PRADS, Changwon, Korea.

Das, K. \& Batra, R. C. 2011. Local water slamming impact on sandwich composite hulls. International Journal of Multiphysics 6(3): 305-339.

Das, S. \& Cheung, K.F. 2012. Hydroelasticity of marine vessels advancing in a seaway. Journal of Fluids and Structures 34: 271-290.

de Hauteclocque, G., Derbanne, Q. \& El Gharbaoui, A. 2012. Comparison of different design waves with spectral analysis. In Proc. $31^{\text {st }}$ Int. Conf. on Ocean, Offshore and Arctic Engineering OMAE, Rio de Janeiro, Brazil.

de Hauteclocque, G., Derbanne, Q. \& Mienahou, T. 2013. Non linearity of extreme vertical bending moment - comparison of design wave approaches and short term approaches. In Proc. $32^{\text {nd }}$ Int. Conf. on Ocean, Offshore and Arctic Engineering OMAE, Nantes, France.

Decò, A. \& Frangopol, D.M. 2013. Risk-informed optimal routing of ships considering different damage scenarios and operational conditions. Reliability Engineering and System Safety 119: 126-140.

Delitala, A.M.S., Gallino, S., Villa, L., Lagouvardos, K. \& Drago, A. 2010. Weather routing in longdistance Mediterranean routes. Theoretical and Applied Climatology 102(1-2):.125-137.

Derbanne, Q., Bigot, F. \& de Hauteclocque, G. 2012a. Comparison of design wave approach and short term approach with increased wave height in the evaluation of whipping induced bending moment. In Proc. $31^{\text {st }}$ Int. Conf. on Ocean, Offshore and Arctic Engineering OMAE, Rio de Janeiro, Brazil.

Derbanne, Q., de Hautecloque, G., El Gharbaoui, A. \& de Belizal, P. 2013a. Design wave selection for strength assessment of floating structures In Proc. $12^{\text {th }}$ Int. Symp. on Practical Design of Ships and Other Floating Structures PRADS, Changwon, Korea.

Derbanne, Q., de Hautecloque, G. \& Mienahou, T. 2013b. Non linearity of extreme vertical bending moment : discussion on the existing rule formulation. In Proc. $32^{\text {nd }}$ Int. Conf. on Ocean, Offshore and Arctic Engineering OMAE, Nantes, France.

Derbanne, Q., Rezende, F., de Hauteclocque, G. \& Chen, X.B. 2011. Evaluation of rule-based fatigue design loads associated at a new probability level. In Proc. $21^{\text {st }}$ Int. Offshore and Polar Engineering Conference ISOPE, Hawaii, USA.

Derbanne, Q., Sireta, F.X., Bigot, F. \& de Hauteclocque, G. 2012b. Discussion on hydroelastic contribution to fatigue damage of containerships. In Proc. $6^{\text {th }}$ Int. Conf. on Hydroelasticity in Marine Technology, Tokyo, Japan.

Dessi, D. 2013a. Reconstruction of the experimental slamming force distribution based on POD. In Proc. $32^{\text {nd }}$ Int. Conf. on Ocean, Offshore and Arctic Engineering OMAE, Nantes, France.

Dessi, D. 2013b. Damping of ship global modes: techniques and analysis. In Proc. $12^{\text {th }}$ Int. Symp. on Practical Design of Ships and Other Floating Structures PRADS, Changwon, Korea.

Dessi, D., 2014b. Load field reconstruction with a combined POD and integral spline approximation technique. Mechanical Systems and Signal Processing 2: 442-467.

Dessi, D. 2014a. Whipping-based criterion for the identification of slamming events. International Journal of Naval Architecture \& Ocean Enginering 6(4).

Dessi, D. \& Ciappi, E. 2013. Slamming clustering on fast ships: From impact dynamics to global response analysis. Ocean Engineering 62: 110-122.

Downes, J., Moore, C., Incecik, A., Stumpf, E. \& McGregor, J. 2007. A method for the quantitative assessment of performance of alternative designs in the accidental condition. In Proc. $10^{\text {th }}$ Int. Symp. on Practical Design of Ships and Other Floating Structures PRADS, Texas, USA.

Drummen, I. \& Holtmann, M. 2014. Benchmark study of slamming and whipping. Ocean Engineering 86: 3-10.

El Malki Alaoui, A., Neme, A., Tassin, A. \& Jacques, N. 2012. Experimental study of coefficients during vertical water entry of rigid shapes at constant speeds. Applied Ocean Research 37: 183-197.

Faltinsen, O.M., Rognebakke, O.F. \& Timokha, A.N. 2003. Resonant three dimensional nonlinear sloshing in a square base basin. Journal of Fluid Mechanics. 487(1): 1-42.

Faltinsen, O.M. \& Timokha, A.N. 2012. On sloshing modes in a circular tank. Journal of Fluid Mechanics 695: 467-477.

Faltinsen, O.M. \& Timokha, A. 2013. Multimodal analysis of weakly nonlinear sloshing in a spherical tank. Journal of Fluid Mechanics 719: 129-164. 
Faltinsen, O.M., Firoozkoohi, R. \& Timokha, A.N. 2011.Steady-state liquid sloshing in a rectangular tank with a slat-type screen in the middle: quasilinear modal analysis and experiments. Physics of Fluids 23: 042101

Fricke, W. \& Paetzold, H. 2012. Experimental investigation of the effect of whipping stresses on the fatigue life of ships. In Proc. Int. Marine Design Conference IMDC, Glasgow, UK.

Fricke, W. \& Paetzold, H. 2013. Experimental investigations on fatigue damage of ship structures caused by whipping stresses. In Proc. $12^{\text {th }}$ Int. Symp. on Practical Design of Ships and Other Floating Structures PRADS, Changwon, Korea.

Fu, X., \& Qin, Z. 2014. Calculation of the added mass matrix of water impact of elastic wedges by the discrete vortex method. Journal of Fluids and Structures 44: 316-323.

Fukasawa, T. 2012. Some considerations on the effect of wave-induced vibrations upon hull-girder fatigue strength of a Post-Panamax container ship. In Proc. $6^{\text {th }}$ Int. Conf. on Hydroelasticity in Marine Technology, Tokyo, Japan.

Fukasawa, T. \& Mukai, K. 2013. On the effects of hull-girder vibration upon fatigue strength of a postpanamax container ship disaggregated by short-term sea state. In Proc. $12^{\text {th }}$ Int. Symp. on Practical Design of Ships and Other Floating Structures PRADS, Changwon, Korea.

Godderidge, B., Turnock, S. R. \& Tan, M. 2012a. A rapid method for the simulation of sloshing using a mathematical model based on the pendulum equation. Computers \& Fluids 57: 163-171.

Godderidge, B., Turnock, S. R. \& Tan, M. 2012b. Evaluation of a rapid method for the simulation of sloshing in rectangular and octagonal containers at intermediate filling levels. Computers \& Fluids 57: $1-24$.

Gotoh, H., Khayyer, A., Ikari, H., Arikawa, T. \& Shimosako, K. 2014. On enhancement of incompressible SPH method for simulation of violent sloshing flows. Applied Ocean Research 46: 104-115.

Gotoh K., Matsuda, K. \& Kitamura, O. 2012. Numerical simulation of fatigue crack propagation under superposed loading histories with two different frequencies. In Proc. $6^{\text {th }}$ Int. Conf. on Hydroelasticity in Marine Technology, Tokyo, Japan.

Greco, M. \& Lugni, C. 2012. 3-D seakeeping analysis with water on deck and slamming Part 1: Numerical solver. Journal of Fluids and Structures 33: 127-147.

Greco, M., Bouscasse, B. \& Lugni, C. 2012. 3-D seakeeping analysis with water on deck and slamming Part 2: Experiments and physical investigation. Journal of Fluids and Structures 33: 148-179.

Greco, M., Colicchio, G., Lugni, C. \& Faltinsen, O.M. 2013. 3D domain decomposition for violent waveship interactions. International Journal for Numerical Methods in Engineering 95(8): 661-684.

Guedes Soares, C. 1996. On the definition of rule requirements for wave induced vertical bending moments. Marine Structures 9: 409-425.

Guevel, P., Bougis, J. \& Hong, D.C. 1979. Formulation du problème des oscillations des corps flottants animés d'une vitesse de route moyenne constante et sollicités par la houle. Summary of 4ème Congrès Français de Mécanique, Nancy, France.

Guo, B.J., Steen, S. \& Deng, G.B. 2012a. Seakeeping prediction of KVLCC2 in head waves with RANS. Applied Ocean Research 35: 56-67.

Guo, L. C., Zhang, S., Morita, K. \& Fukuda, K. 2012b. Fundamental validation of the finite volume particle method for 3d sloshing dynamics. International Journal for Numerical Methods in Fluids 68(1): 117.

Hanninen, S.K., Mikkola, T. \& Matusiak, J. 2012. On the numerical accuracy of the wave load distribution on a ship advancing in short and steep waves. Journal of Marine Science and Technology 17(2): 125-138.

Hanninen, S.K., Mikkola, T. \& Matusiak, J. 2014. Computational and experimental study on local ship loads in short and steep waves. Journal of Marine Science and Technology 19(1): 103-115.

He, G. \& Kashiwagi, M. 2014. A time-domain higher-order boundary element method for 3D forwardspeed radiation and diffraction problems. Journal of Marine Science and Technology 19(2): 228-244.

Hirdaris, S., Argiryiadis, K., Bai, W., Dessi, D., Ergin. A., Fonseca, N., Gu, X., Hermundstad, O.A., Huijsmans, R., Iijima, K., Nielsen, U.D., Papanikolau, A., Parunov, J. \& Incecik, A. 2014. Loads for use in the design of ships and offshore structures. Ocean Engineering 78: 131-174.

Hong, D.C. 2000. Application of the improved Green integral equation to the radiation-diffraction problem for a floating ocean structure in waves and current. International Journal of Ocean Engineering \& and Technology 3(1): 14-22.

Hong, D.C., Hong, S.Y., Lee, G.J \& Shin, M.S. 2014. Influence of the waterline integral on the solution of the frequency-domain forward-speed radiation-diffraction problem of a ship advancing in waves. In Proc. $33^{\text {rd }}$ Int. Conf. on Ocean, Offshore and Arctic Engineering OMAE, San Francisco, USA.

Hong, D.C., Hong, S.Y., Sung, H.G. \& Nam, B.W. 2013b. Influence of the Waterline Integral on the Solution of the Time-Domain Forward-Speed Radiation-Diffraction Problem. In Proc. $12^{\text {th }}$ Int. Symp. on Practical Design of Ships and Other Floating Structures PRADS, Changwon, Korea. 
Hong, S.Y., Kim, B.W. and Nam, B.W. 2012. Experimental study on torsion springing and whipping of large containership. Int. Journal of Offshore and Polar Engineering 22:2, 97-107.

Hong, S.Y. and Kim, B.W. (2014). Experimental investigations of higher-order springing and whippingWILS project. Int. Journal of Naval Architecture and Ocean Engineering 6, 1160-1181.

Hu, Z., Tang, W., Xue, H. \& Ren, S. 2014. Response of beams under the impact of freak waves. In Proc. $33^{\text {rd }}$ Int. Conf. on Ocean, Offshore and Arctic Engineering OMAE, San Francisco, USA.

Hu, Z. Z., Causon, D. M., Mingham, C. G. \& Qian, L. 2013. A Cartesian cut cell free surface capturing method for 3D water. International Journal for Numerical Methods in Engineering 71: 1238-1259.

Hua, C., Fang, C. \& Cheng, J. 2011. Simulation of fluid-solid interaction on water ditching of an airplane by ALE method. Journal of Hydrodynamics 23(5): 637-642.

IACS. 2001. Recommendation 34: Standard Wave Data. International Association of Classification Societies.

IACS. 2006a. Common Structural Rules for Bulk Carriers. International Association of Classification Societies.

IACS. 2006b. Common Structural Rules for Double Hull Oil Tankers. International Association of Classification Societies.

IACS. 2010. Unified Requirement S11: Longitudinal Strength Standard. International Association of Classification Societies.

IACS. 2013. Common Structural rules for Bulk Carriers and Oil Tankers. International Association of Classification Societies.

Iafrati, A., Grizzi, S., Siemann, M. \& Benìtez Montanés, L. 2014. Experimental analysis of the water entry of a plate at high horizontal speed. In Proc. $30^{\text {th }}$ Symp. Naval Hydrodynamics, Tasmania, Australia.

Iijima, K., Suzaki, Y. \& Fujikubo, M. 2014. Scaled model tests for the post-ultimate strength collapse whipping loads behaviour of a ship's hull girder under whipping loads. Ships and Offshore Structures DOI: 10.1080/17445302.2013.870774.

Ikeda, T., Ibrahim, R. A., Harata, Y. \& Kuriyama, T. 2012. Nonlinear liquid sloshing in a square tank subjected to obliquely horizontal excitation. Journal of Fluid Mechanics 700: 304-328.

Ikeda, C.M. \& Judge, C.Q. 2014. Slamming impacts of hydrodynamically supported craft. In Proc. $33^{\text {rd }}$ Int. Conf. on Ocean, Offshore and Arctic Engineering OMAE, San Francisco, USA.

IMO. 2012. Development of measures to prevent loss of containers - verification of container weight; submission by Denmark, Netherlands, the United States, BIMCO, the International Association of Port and Harbours (IAPH), the International Chamber of Shipping (ICS), the International Transport Workers Federation (ITF) and the World Shipping Council (WSC). International Maritime Organisation IMO DSC 17/INF.5.

IMO. 2014a. Interim report on large containership safety in response to the loss of MV MOL COMFORT. International Maritime Organisation MSC 93/INF.14.

IMO. 2014b. Maritime Safety Committee (MSC), 93 ${ }^{\text {rd }}$ session, 14 to 23 May 2014. International Maritime Organisation.

Ishii E., Kobayashi, E., Mizunoe, T. \& Maki, A. 2010. Proposal of new-generation route optimization technique for an oceangoing vessel. In Proc. of OCEANS, Seattle, USA.

ISSC. 2012a. Report of Committee V.7 Impulsive Pressure Loading and Response Assessment. In Proc. $18^{\text {th }}$ Int. Ships and Offshore Structures Congress, Rostock, Germany 2: 275 - 330.

ISSC. 2012b. Report of Technical Committee I.2 Loads. In Proc. $18^{\text {th }}$ Int. Ships and Offshore Structures Congress, Rostock, Germany 1:79-150

ISSC. 2015a. Report of Technical Committee I.2 Loads. In Proc. $19^{\text {th }}$ Int. Ships and Offshore Structures Congress, Cascais, Portugal

ISSC. 2015b. Report of Technical Committee I.1 Environment. In Proc. $19^{\text {th }}$ Int. Ships and Offshore Structures Congress, Cascais, Portugal

Jacobi, G., Thomas, G., Davis, M.R. \& Davidson, G. 2014. An insight into the slamming behaviour of large high-speed catamarans through full-scale measurements. Journal of Marine Science \& Technology 19:15-32.

Ji, Y. M., Shin, Y. S., Park, S. J. \& Hyun, M. J. 2012. Experiments on non-resonant sloshing in a rectangular tank with large amplitude lateral oscillation. Ocean Engineering 50: 10-22.

Jin, H., Liu, Y., Li, H.-J. 2014. Experimental study on sloshing in a tank with an inner horizontal perforated plate. Ocean Engineering 82: 75-84.

Joga, R., Saripilli, J., Dhavalikar, S. \& Kar, A. 2014 Numerical simulations to compute rate of water ingress into open holds due to green waters. In Proc. $24^{\text {th }}$ Int. Offshore and Polar Engineering Conference ISOPE, Busan, Korea.

Johannessen, T.B. \& Hagen, Ø. 2012. Estimating design levels for strongly nonlinear response. In Proc. $31^{\text {st }}$ Int. Conf. on Ocean, Offshore and Arctic Engineering OMAE, Rio de Janeiro, Brazil.

Journée, J.M.J. 1992. Experiments and Calculations on 4 Wigley Hull Forms in Head Waves. Technical report 0909, Ship Hydromechanics Lab. DUT, The Netherlands. 
Juncher Jensen, J. 2011. Extreme value predictions using Monte Carlo simulations with artificially increased load spectrum. Journal of Probabilistic Engineering Mechanics 26: 399-404.

Jung, J., Yoon, H., Lee, C. \& Shin, S. 2012. Effect of the vertical baffle height on the liquid sloshing in a three-dimensional rectangular tank. Ocean Engineering 44: 79-89.

Kashiwagi, M. \& Hara, T. 2012. A method for ship hydroelasticitic analysis by means of Rankine panel method. In Proc. $6^{\text {th }}$ Int. Conf. on Hydroelasticity in Marine Technology, Tokyo, Japan.

Khabakhpasheva, T. I. \& Korobkin, A. A. 2013. Elastic wedge impact onto a liquid surface: Wagner's solution. Journal of Fluids and Structures 36: 32-49.

Kim, K.H., Bang, J.S., Kim. J.H., Kim, Y., Kim, S.J. \& Kim, Y. 2013a. Fully coupled BEM-FEM analysis for ship hydroelasticity in waves. Marine Structures 33: 71-99.

Kim, D.H., Engle, A.H. \& Troesch, A.W. 2012a. Estimates of long-term combined wave bending and whipping for two alternative hull forms. Transactions Society of Naval Architects and Marine Engineers SNAME 1-30.

Kim, Y. \& Hermansky, G. 2014. Uncertainties in seakeeping analysis and related loads and response procedures. Ocean Engineering, 86: 68-81.

Kim, K.H. \& Kim, Y. 2012. Numerical analysis on ship hydroelasticity by using 3d Rankine panel method and $3 \mathrm{~d}$ finite element method. In Proc. $6^{\text {th }}$ Int. Conf. on Hydroelasticity in Marine Technology, Tokyo, Japan.

Kim, S., Kim, C.Y. \& Cronin, D. 2013c .Green water impact loads on breakwaters of large container carriers. In Proc. $12^{\text {th }}$ Int. Symp. on Practical Design of Ships and Other Floating Structures PRADS, Changwon, Korea.

Kim, Y., Kim, J.H. \& Kim, Y. 2013d. Whipping identification of a flexible ship using wavelet crosscorrelation. Ocean Engineering 74: 90-100.

Kim, J.H., Kim, K.H., Jung, B.H., Choi , J.H. \& Kim, Y. 2012b. Analysis on ship springing using fullycoupled fsi models. In Proc. $6^{\text {th }}$ Int. Conf. on Hydroelasticity in Marine Technology, Tokyo, Japan.

Kim, K.S., Kim, M.H. \& Park, J.C. 2014. Simulation of multi-liquid-layer sloshing with vessel motion by using moving particle simulation. In Proc. $33^{\text {rd }}$ Int. Conf. on Ocean, Offshore and Arctic Engineering $O M A E$, San Francisco, USA.

Kim, Y., Kim, S.Y., Ahn, Y.J. \& Kim, K.H. 2013b. Experimental analysis on sloshing loads for LNG cargo design. In Proc. $12^{\text {th }}$ Int. Symp. on Practical Design of Ships and Other Floating Structures PRADS, Changwon, Korea.

Kim, S.J. \& Paik, J.K. 2013. Advanced method for ship structural design under slamming impact pressure loads. In Proc. $12^{\text {th }}$ Int. Symp. on Practical Design of Ships and Other Floating Structures PRADS, Changwon, Korea.

Kim, Y. \& Park, S.G. 2014. Wet damping estimation of the scaled segmented hull model using the random decrement technique. Ocean Engineering 75: 71-80.

Kleefsman, K.M.T. 2005. Water Impact Loading on Offshore Structures: A Numerical Study. PhD Thesis, University of Groningen.

Kobayakawa, H., Kusumoto, H., Nagashima, T. \& Neki, I. 2012. Hydroelastic response analysis using unsteady time domain analysis of ship motions. In Proc. $6^{\text {th }}$ Int. Conf. on Hydroelasticity in Marine Technology, Tokyo, Japan.

Koh, C., Luo, M., Gao, M. \& Bai, W. 2013. Modelling of liquid sloshing with constrained floating baffle. Computers \& Structures 122: 270-279.

Koning, J. 2009. lashing@sea, Executive Summary. SMIG07002 Report No: 19717-20-TM. Maritime Research Institute The Netherlands, Senter Novem.

Koo, J.B., Kim, B.J., Jang, K.B., Suh, Y.S. \& Bigot, F. 2013. Fatigue assessment of the 18,000 TEU container vessel considering the effect of springing. In Proc. $23^{\text {rd }}$ Int. Offshore and Polar Engineering Conference ISOPE, Alaska, USA.

Korobkin, A. A. 2013. A linearized model of water entry. Journal of Fluid Mechanics 737: 368-386.

Korobkin, A.A., Guéret, E., \& Malenica, S. 2006. Hydroelastic coupling of beam finite element model with Wagner theory of water impact. Journal of Fluids and Structures 22: 493-504.

Lavroff, J., Davis, M. R., Holloway, D. S. \& Thomas, G. 2013. Wave slamming loads on wave-piercer catamarans operating at high-speed determined by hydro-elastic segmented model experiments. $M a$ rine Structures 33: 120-142.

Lee, Y., Chan, H.-S., Pu, Y., Incecik, A. \& Dow, R. S. 2012c. Global wave loads on a damaged ship. Ships and Offshore Structures 7(3): 237-268.

Lee T., Chung, H. \& Myung, H. 2011 .Multi-resolution path planning for marine surface vehicle considering environmental effects. In Proc. of OCEANS, Santander, Spain.

Lee, H.H., Lim, H.J. \& Rhee, S.H. 2012b. Experimental investigation of green water on the deck for a CFD validation database. Ocean Engineering 42: 47-60. 
Lee, Y., White, N., Wang, Z. \& Park, J.B. 2012a. Whipping responses and whipping effects on design bending moments of a large container ship. In Proc. $6^{\text {th }}$ Int. Conf. on Hydroelasticity in Marine Technology, Tokyo, Japan.

Li, H.-T., Li, J., Zong, Z. \& Chen, Z. 2014a. Numerical studies on sloshing in rectangular tanks using a tree-based adaptive solver and experimental validation. Ocean Engineering 82: 20-31.

Liu, S., Papanikolaou, A. \& Zaraphonitis, G. 2014a. Time domain simulation of nonlinear ship motions using an impulse response function method. In Proc. Int. Conf. on Maritime Technology ICMT.

Lin, Y.H., Fang, M.C. \& Yeung, R.W. 2013. The optimization of ship weather-routing algorithm based on the composite influence of multi-dynamic elements. Applied Ocean Research 43: 184-194.

Lloyd's Register. 2011. Guidance Notes on the Assessment of Global Design Loads of Large Container Ships and Other Ships Prone to Whipping and Springing. Draft Version 1.1.

Lu, H., Yang, C. \& Loehner, R. 2012. Numerical studies of green water impact on fixed and moving bodies. International. Journal of Offshore and Polar Engineering 22(2): 123-132.

Lundgren, H. 1969. Wave shock forces: An analysis of deformations and forces in the wave and the foundation. In Proc. Research on Wave Action. Symp., Delft, The Netherlands.

Lv, J. \& Grenestedt, J. L. 2013. Some analytical results for the initial phase of bottom slamming. Marine Structures 34: 88-104.

MAIB. 2008. Report on the Investigation of the Structural Failure of MSC Napoli, English Channel on 18 January 2007. Marine Accident Investigation Branch, Southampton, United Kingdom.

MAIB. 2006. Report on the Investigation of the Loss of Cargo Containers overboard from P\&O Nedlloyd Genoa North Atlantic Ocean on 27 January 2006. Report No 20/2006, Marine Accident Investigation Branch, Southampton, United Kingdom.

Maki, A., Akimoto, Y., Nagata, Y., Kobayashi, S., Kobayashi, E., Shiotani, S., Ohsawa, T. \& Umeda, N. 2011. A new weather-routing system that accounts for ship stability based on a real-coded genetic algorithm. Journal of Marine Science and Technology 16(3): 311-322.

Malenica, S. \& Derbanne, Q. 2012. Hydro-elastic issues in the design of ultra large container ships-tulcs project. In Proc. $6^{\text {th }}$ Int. Conf. on Hydroelasticity in Marine Technology, Tokyo, Japan.

Mao, W., Li, Z., Ringsberg, J.W. \& Rychlik, I. 2012. Application of a ship-routing fatigue model to case studies of 2800 TEU and 4400 TEU container vessels. Journal of Engineering for the Maritime Environment 226(3): 222-234.

Mao, W., Ringsberg, J.W., Rychlik, I. \& Storhaug, G. 2010. Development of a fatigue model useful in ship routing design. Journal of Ship Research 54(4): 281-293.

Mao, W. \& Rychlik, I. 2012. Estimation of extreme ship response. Journal of Ship Research 56(1): 23-34.

Mariani, R. \& Dessi, D. 2012. Analysis bending modes of floating structure by POD. Journal of Fluids and Structures 28: 115-134.

Masoud, H. 2013. Nonlinear Wave Load on Decks of coastal Structures. PhD dissertation, University of Hawai'I, Manoa, USA.

Miao, S.H., Temarel, P. \& Hirdaris, S.E. 2012. The antisymmetric dynamic behaviour of a modern containership in regular waves. In Proc. $6^{\text {th }}$ Int. Conf. on Hydroelasticity in Marine Technology, Tokyo, Japan.

Mirciu, I., Rubanenco, I. \& Domnisoru, L. 2012. On the numerical non-linear hydroelastic response of a lpg 100,000 cbm carrier in irregular head waves In Proc. $6^{\text {th }}$ Int. Conf. on Hydroelasticity in Marine Technology, Tokyo, Japan.

Mitra, S., Wang, C., Reddy, J. \& Khoo, B. 2012. A 3d fully coupled analysis of nonlinear sloshing and ship motion. Ocean Engineering 39, 1-13.

Moan, T., Shu, Z., Drummen I. \& Almashi, H. 2006. Comparative reliability analysis of ships - considering different ship types and the effect of ship operations on loads. Transactions Society of Naval Architects and Marine Engineers SNAME 114: 16-54.

Mohammed, E.A., Chan, H.S. \& Hirdaris, S.E. 2012. Global wave load combinations by cross-spectral methods. Marine Structures 29: 131-151.

Molin, B. \& Remy, F. 2013. Experimental and numerical study of the sloshing motion in a rectangular tank with a perforated screen. Journal of Fluids and Structures 43: 463-480.

Molin, B., Remy, F., Audiffren, C. \& Marcer, R. 2012. Experimental and numerical study of liquid sloshing in a rectangular tank with three tluids. In Proc. $22^{\text {nd }}$ Int. Offshore and Polar Engineering Conference ISOPE, Rhodes, Greece.

Moore, M. R., Howison, S. D., Ockendon, J. R. \& Oliver, J. M. 2012. Three-dimensional oblique waterentry problems at small deadrise angles. Journal of Fluid Mechanics 711: 259-280.

Moore, M. R., Howison, S. D., Ockendon, J. R. \& Oliver, J. M. 2013. A note on oblique water entry. Journal of Engineering Mathematics 81: 67-74.

Nguyen, V.T., Vu, D.T., Park, W.G. \& Jung, Y.R. 2014. Numerical analysis of water impact forces using a dual-time pseudo-compressibility method and volume-of-fluid interface tracking algorithm. Computer \& Fluids 103: 18-33. 
Nichols, J.M., Fackler, P.L., Pacifici, K., Murphy, K.D. \& Nichols, J.D. 2014. Reducing fatigue damage for ships in transit through structured decision making. Marine Structures 38: 18-43.

Nielsen, U.D., Andersen, I.M.V. \& Koning, J. 2013. Comparisons of means for estimating sea states from an advancing large container ship. In Proc. $12^{\text {th }}$ Int. Symp. on Practical Design of Ships and Other Floating Structures PRADS, Changwon, Korea.

Nielsen, J.K. \& Michelsen, J. 2007. Data Models for Ship Varying Data Project: ADOPT. Document ID: ADOPT-WP4.2-DEL-2007-03-21-FINAL-Data_Models_Ship_Varying_Data-FORCE Technology.

Oberhagemann, J. and El Moctar, O. 2012. Numerical and experimental investigations of whipping and springing of ship structures. Int. Journal of Offshore and Polar Engineering 22:2, 108-114.

Oberhagemann, J., Shigunov, V. \& El Moctar, O. 2012. Application of CFD in long-term extreme value analyses of wave loads. Ship Technology Research 59(3): 4-22.

Ogawa, Y., Kitamura, O. \& Toyoda, M. 2012 A study for the statistical characteristic of slamming induced vibration of large container ship In Proc. $6^{\text {th }}$ Int. Conf. on Hydroelasticity in Marine Technology, Tokyo, Japan.

Ogilvie, T. F. 1983. Second-order hydrodynamic effects on ocean platforms. Int. Wrkshp on Ship and Platform Motions, University of California, Berkeley, CA.

Onorato, M., Proment, D., Clauss, G. \& Klein, M. 2013. Rogue waves: From nonlinear Schrödinger breather solutions to seakeeping test. Public Library of Science PLOS One 8(2) e54629.

Osawa, N., Nakamura, T., Yamamoto, N. \& Sawamura, J. 2013. Experimental study on high frequency effect on fatigue by using the new high speed fatigue testing machine. In Proc. $12^{\text {th }}$ Int. Symp. on Practical Design of Ships and Other Floating Structures PRADS, Changwon, Korea.

Paik, J.K., Lee, S.E., Kim, B.J., Seo, J.K., Ha, Y.C., Matsumoto, T. \& Kim, D.H. 2014. A new method for determining the design sloshing loads for LNG FPSOs. In Proc. $33^{\text {rd }}$ Int. Conf. on Ocean, Offshore and Arctic Engineering OMAE, San Francisco, USA.

Pakozdi, C., Östman, A., Stansberg, C.T. \& Carvalho, D.F. 2014. Green water on FPSO analyzed by a coupled Potential-Flow-NS-VOF method. In Proc. $33^{\text {rd }}$ Int. Conf. on Ocean, Offshore and Arctic Engineering $O M A E$, San Francisco, USA.

Panciroli, R. Abrate, S. \& Minak, G. 2013. Dynamic response of flexible wedges entering the water. Composite Structures 99: 163-171.

Panciroli, R., Abrate, S., Minak, G. \& Zucchelli, A. 2012. Hydroelasticity in water-entry problems: Comparison between experimental and SPH results. Composite Structures 94: 532-539.

Panigrahi, J.K., Padhy, C.P., Sen, D., Swain, J. \& Larsen, O. 2012. Optimal ship tracking on a navigation route between two ports: A hydrodynamics approach. Journal of Marine Science and Technology 17(1): 59-67.

Papaioannou, I., Gao, R.P., Rank, E. \& Wang, C.M. 2013. Stochastic hydroelastic analysis of pontoontype very large floating structures considering directional wave spectrum. Probabilistic Engineering Mechanics 33: 26-37.

Papanikolaou, A., Mohammed, E.A. \& Hirdaris, S.E. 2014 Stochastic uncertainty modelling for ship design loads and operational guidance. Ocean Engineering 86: 47-57.

Parunov, J., Ćorak, M. and Gledić, I. 2015. Comparison of two practical methods for seakeeping assessment of damaged ships. In Proc. $5^{\text {th }}$ Int. Conf. on Marine Structures: Analysis and Design of Marine Structures, Guedes Soares, C., Shenoi, R. A. (eds). London : Taylor \& Francis Group, 2015. 37 44.

Parunov, J., Andrić, J., Ćorak, M. \& Kitarović, S. 2014. Structural reliability assessment of container ship at the time of accident. Journal of Engineering for the Maritime Environment.

Piro, D. J. \& Maki, K. 2011. Hydroelastic wedge entry and exit. In Proc. $11^{\text {th }}$ Int. Conf. On Fast Sea Transportation, Hawaii, USA.

Piro, D. J. \& Maki, K. 2012. Water exit of a wedge-shaped body. In Proc. $27^{\text {th }}$ Int. Wkshp. on Water Waves \& Floating Bodies IWWWFB, Copenhagen, Denmark.

Piro, D.J. \& Maki, K.J. 2013. Hydroelastic analysis of bodies that enter and exit water. Journal of Fluids and Structures 37: 134-150.

Pistani, F. \& Thiagarajan, K. 2012. Experiments on non-resonant sloshing in a rectangular tank with large amplitude lateral oscillation. Ocean Engineering 52: 60-74.

Prasetyo, F.A., Osawa, N. \& Kobayashi, T. 2012. Study on preciseness of load history generation based on storm model for fatigue assessment of ship structure members. In Proc. $22^{\text {nd }}$ Int. Offshore and Polar Engineering Conference ISOPE, Rhodes, Greece.

Prestileo, A., Rizzuto, E., Teixeira, A.P. \& Guedes Soares, C. 2013. Bottom damage scenarios or the hull girder structural assessment. Marine Structures 33: 33-55.

Rajendran, S., Fonseca, N. \& Guedes Soares, C. 2013. Estimation of short term probability distributions of wave induced loads acting on a cruise vessel in extreme seas. In Proc. $32^{\text {nd }}$ Int. Conf. on Ocean, Offshore and Arctic Engineering OMAE, Nantes, France. 
Rajendran, S., Fonseca, N. \& Guedes Soares, C. 2014. Prediction of ship responses in large amplitude waves using a body nonlinear time domain method with 2nd order Froude-Krylov pressure. . In Proc. $33^{\text {rd }}$ Int. Conf. on Ocean, Offshore and Arctic Engineering OMAE, San Francisco, USA.

Rathje, H., Kahl, A. \& Schellin, T.E. 2012. Semi-empirical assessment of long-term high-frequency hull girder response of containerships. In Proc. $22^{\text {nd }}$ Int. Offshore and Polar Engineering Conference ISOPE, Rhodes Greece.

Reinhard, M., Korobkin, A. A. \& Cooker, M. J. 2012a. The bounce of a blunt body from a water surface at high horizontal speed. In Proc. $27^{\text {th }}$ Int. Wkshp. on Water Waves \& Floating Bodies IWWWFB, Copenhagen, Denmark.

Reinhard, M., Korobkin, A. A. \& Cooker, M. J.,2012b. Elastic plate impact intowater at high horizontal speed with early water detachment. In Proc. $6^{\text {th }}$ Int. Conf. on Hydroelasticity in Marine Technology, Tokyo, Japan.

Reinhard, M., Korobkin, A. A. \& Cooker, M. J. 2013. Water-entry of an elastic plate at high horizontal speed. Journal of Fluid Mechanics 724: 123-153.

Ruggeri, F., Wata, R.A., Brisson, H., Mello, P.A., Sampaio Carvalho e Silva, C.M.P. \& Vieira, D.P. 2013. Numerical prediction of green water events in beam seas. In Proc. $12^{\text {th }}$ Int. Symp. on Practical Design of Ships and Other Floating Structures PRADS, Changwon, Korea.

Santos, F. M., Casetta, L. \& Pesce, C. P. 2012. Application of a variational method to the vertical hydrodynamic impact of axisymmetric bodies. Applied Ocean Research 39: 75-82.

Sarala, R., Hajiarab, M. \& Bamford, R. 2011. Equivalent Design Wave approach for calculating sitespecific environmental loads on an FPSO. In Proc. $30^{\text {th }}$ Int. Conf. on Ocean, Offshore and Arctic Engineering OMAE, Rotterdam, The Netherelands.

Schiller, R.V., Pâkozdi, C., Stansberg, C.T. \& Carvalho, D.F. 2014a. Green water and wave impact on FPSOs in Santos Basin: Challenges and prediction tools. In Proc. $33^{\text {rd }}$ Int. Conf. on Ocean, Offshore and Arctic Engineering OMAE, San Francisco, USA.

Scolan, Y.M. \& Korobkin, A. A. 2012. Hydrodynamic impact (Wagner) problem and Galin's theorem. In Proc. $27^{\text {th }}$ Int. Wkshp. on Water Waves \& Floating Bodies IWWWFB, Copenhagen, Denmark.

Scolan, Y.M. \& Korobkin, A. A. 2013. Hydrodynamic impact of three-dimensional bodies on waves. In Proc. $27^{\text {th }}$ Int. Wkshp. on Water Waves \& Floating Bodies IWWWFB, Marseille, France.

Seng, S. \& Juncher Jensen, J. 2012. Slamming simulations in a conditional wave. In Proc. $31^{\text {st }}$ Int. Conf. on Ocean, Offshore and Arctic Engineering OMAE, Rio de Janeiro, Brazil.

Seng, S. \& Juncher Jensen, J. 2013. An application of a free surface CFD method in the short-term extreme response analysis of ships. In Proc. $12^{\text {th }}$ Int. Symp. on Practical Design of Ships and Other Floating Structures PRADS, Changwon, Korea.

Seng, S., Juncher Jensen, J. and Malenica, S. 2014. Global hydroelastic model for springing and whipping based on a free-surface CFD Code (OpenFOAM). International Journal of Naval Architecture \& Ocean Engineering 6: 1024-1040.

Senjanović, I., Hadžić, N. \& Vladimir, N. 2012. Improved methodology of ship hydroelastic analysis. In Proc. $6^{\text {th }}$ Int. Conf. on Hydroelasticity in Marine Technology, Tokyo, Japan.

Shao, J., Li, H., Liu, G. \& Liu, M. 2012. An improved SPH method for modeling liquid sloshing dynamics. Computers \& Structures 100: 18-26.

Simonsen, C.D., Otzen, J.F., Joncquez, S. \& Stern, F. 2013 EFD and CFD for KCS heaving and pitching in regular head waves. Journal of Marine Science and Technology 18(4): 435-459.

Song, K.H., Kim, Y. \& Park, D.M. 2013. Quantitative and qualitative analyses of parametric roll for ship design and operational guidance. Journal of Engineering for the Maritime Environment 227(2): 117189.

Spanos, D. A. \& Papanikolaou, A.D. 2012. On the time dependence of survivability of ROPAX ships. Journal of Marine Science and Technology 17: 40-46.

Stettler, J. W. \& Thomas, B.S. 2012. Flooding and structural forensic analysis of the sinking of the RMS Titanic. Transactions Society of Naval Architects and Marine Engineers SNAME 120.

Storhaug, G. 2009 . The 4400 TEU container vessel MSC Napoli broke its back, but did whipping contribute? In Proc. $5^{\text {th }}$ Int. Conf. on Hydroelasticity in Marine Technology, Southampton, UK.

Storhaug, G. 2012. The effect of heading on springing and whipping induced fatigue damage measured on container vessels. In Proc. $6^{\text {th }}$ Int. Conf. on Hydroelasticity in Marine Technology, Tokyo, Japan.

Storhaug, G., Pettersen, T.A., Oma, N. \& Blomberg, B. 2012. The effect of wave induced vibrations on fatigue loading and the safety margin against collapse on two LNG vessels. In Proc. $6^{\text {th }}$ Int. Conf. on Hydroelasticity in Marine Technology, Tokyo, Japan.

Sumi, Y., Yajima, H., Toyosada, M., Yoshikawa, T., Aihara, S., Gotoh, K., Ogawa, Y., Matsumoto, M., Hirota, K., Hirasawa, H., Toyoda, M. \& Morikage, Y. 2013. Fracture control of extremely thick welded steel plates applied to the deck structure of large container ships. Journal of Marine Science and Technology 18: 497-514. 
Sun, H. \& and Faltinsen, O.M. 2012. Hydrodynamic forces on a semi-displacement ship at high speed. Applied Ocean Research 34: 68-77.

Sun, S.Y., Sun, S.L. \& Wu, G.X. 2015. Oblique water entry of a wedge into waves with gravity effect. Journal of Fluids and Structures 52:49-64.

Takahara, H. \& Kimura, K. 2012. Frequency response of sloshing in an annular cylindrical tank subjected to pitching excitation. Journal of Sound \& Vibration 331(13): 3199-3212.

Takahara, H., Kensuke, H. \& Ishida, T. 2012. Nonlinear liquid oscillation in a cylindrical tank with an eccentric core barrel. Journal of Fluids and Structures 35:120-132.

Tassin, A., Jacques, N., El Malki Alaoui, A., Neme, A., Leblè, B. 2012b. Hydrodynamic loads during water impact of 3D solids: Modelling and experiments. Journal of Fluids and Structures 28: 211-231.

Tassin, A., Korobkin, A. A. \& Cooker, M. J. 2012a. Modelling of the oblique impact of an elongated body by $2 \mathrm{D}+\mathrm{t}$ approach. In Proc. $27^{\text {th }}$ Int. Wkshp. on Water Waves \& Floating Bodies IWWWFB, Copenhagen, Denmark.

Tassin, A., Piro, D. J., Korobkin, A. A., Maki, K. \& Cooker, M. J. 2013. Two-dimensional water entry and exit of a body whose shape varies in time. Journal of Fluids and Structures 28: 211-231.

Teixeira A.P., Guedes Soares, C., Chen, N.Z. \& Wang, G. 2013. Uncertainty analysis of load combination factors for global longitudinal bending moments of double-hull tankers. Journal of Ship Research 57(1): 42-58.

Thomas, G., Davis, M.R., Holloway, D.S. \& Roberts, T. 2003. Transient slam response of large high speed catamarans. In Proc. $7^{\text {th }}$ Int. Conf. On Fast Sea Transportation, Ischia, Italy.

Topliss, M.E., Cooker, M.J. \& Peregrine,D.H. 1992. Pressure oscillations during wave impact on vertical walls. In Proc. $23^{\text {rd }}$ Int. Conf. on Coastal Engineering, Venice, Italy.

Toyoda, K., Matsumoto, T., Yamamoto, N. \& Terai, K. 2012. Simplified fatigue assessment considering the occurrence probability of hydro-elastic response in actual sea state conditions. In Proc. $6^{\text {th }}$ Int. Conf. on Hydroelasticity in Marine Technology, Tokyo, Japan.

Tsai, S.C., Hsu, S.H., Chien, H.L., Chou, C.M., Malenica, M. \& Chen, X.B. 2013. Numerical study on seakeeping-sloshing coupling effect of container ship for sea trial purpose. In Proc. $12^{\text {th }}$ Int. Symp. on Practical Design of Ships and Other Floating Structures PRADS, Changwon, Korea.

Tuitman, J. T., Bosman, T. N. \& Harmsen, E. 2013. Local structural response to seakeeping slamming loads. Marine Structures 33: 214-237.

Tveitnes, T., Fairlie-Clark, A.C. \& Varyani, K. 2008. An experimental investigation into the constant velocity water entry of wedge-shaped sections. Ocean Engineering 35(14-15): 1463-1478.

Vanem, E., Bitner-Gregersen, E., Eide, L.I., Garre, L. \& Friis Hansen, P. 2014. Uncertainties of climate modeling and effects on wave induced bending moment SNAME Annual Meeting, Houston.

Vasquez, G., Fonseca, N. \& Guedes Soares, C. 2013. Experimental and numerical extreme motions and vertical bending moments induced by abnormal waves on a bulk carrier. In Proc. $32^{\text {nd }}$ Int. Conf. on Ocean, Offshore and Arctic Engineering OMAE, Nantes, France.

Vidic Perunovic, J. 2012. Ship springing response in finite water depth. Journal of Ship Research 56(2): 80-90.

Wada, R., Iijima, K., Kimura, K., Xu W. \& Fujikubo, M. 2010. Development of a design methodology for a scaled model for hydro-elastoplasticity of a hull girder in waves. In: $4^{\text {th }}$ PAAMES Meeting, Singapore.

Wang, S. \& Guedes Soares, C. 2013. Slam induced loads on bow flared sections with various roll angles. Ocean Engineering 67: 45-57.

Wang, X.L., Gu, X.K. \& Hu, J.J. 2013. Investigation of springing influence on fatigue damage of ship structures. In Proc. $12^{\text {th }}$ Int. Symp. on Practical Design of Ships and Other Floating Structures PRADS, Changwon, Korea.

Wang, X.L., Gu, X.K., Hu, J.J. \& Xu, C. 2012. A study of sloshing influence on wave induced responses of a LNG ship by experimental method. In Proc. $6^{\text {th }}$ Int. Conf. on Hydroelasticity in Marine Technology, Tokyo, Japan.

Wu, C.H., Faltinsen, O. M. \& Chen, B.F. 2012a. Numerical study of sloshing liquid in tanks with baffles by time-independent finite difference and fictitious cell method. Computers \& Fluids 63: 9-26.

Wu, M.K., Lehn, E. \& Moan, T. 2012b. Design of segmented model for ship seakeeping tests with hydroelastic effects. In Proc. $6^{\text {th }}$ Int. Conf. on Hydroelasticity in Marine Technology, Tokyo, Japan.

Xiao, J. \& Batra, R. C. 2012. Local slamming of curved rigid hulls. International Journal of Multiphysics 6(3): 305-339.

Xiao, J. \& Batra, R. C. 2014. Delamination in sandwich panels due to local water slamming loads. Journal of Fluids and Structures 48: 122-155.

Xiao, L.F., Tao, L.B., Yang, J.M. \& Li, X. 2014. An experimental investigation on wave run-up along the broadside of a single point moored FPSO exposed to oblique waves. Ocean Engineering 88: 81-90.

$\mathrm{Xu}$, H.H. 2013. Numerical Simulation of breaking Wave Impact on the Structure. PhD dissertation, National University of Singapore, Singapore. 
Yang, J., Kim, S., Park, J.S., Jung, B.H. \& Lee, T. 2013. Numerical analysis for slamming impact loads and dynamic structural responses of a containership. In Proc. $12^{\text {th }}$ Int. Symp. on Practical Design of Ships and Other Floating Structures PRADS, Changwon, Korea.

Yang, Q. \& Qiu, W. 2012. Numerical simulation of water impact for 2D and 3D bodies. Ocean Engineering 43: 82-89.

Zhao, W., Key, S., Yang, J., Tao, L. \& White, D. 2014b. Research on the coupling effects between ship motions and sloshing. In Proc. $33^{\text {rd }}$ Int. Conf. on Ocean, Offshore and Arctic Engineering OMAE, San Francisco, USA.

Zhao, W., Yang, J., Hu, Z. \& Tao, L. 2014c. Coupled analysis of nonlinear sloshing and ship motions. Applied Ocean Research 47: 85-97.

Zhao, X. \& Hu, C. 2012 Numerical and experimental study on a 2-D floating body under extreme wave conditions. Applied Ocean Research 35: 1-13.

Zhao, X., Ye, Z., Fu, Y. \& Cao, F. 2014a. A CIP-based numerical simulation of freak wave impact on a floating body. Ocean Engineering 87: 50-63.

Zhao, X.Z., Ye, Z.T. \& Fu, Y.N. 2014d. Green water loading on a floating structure with degree of freedom effects. Journal of Marine Science and Technology 19(3): 302-313.

Zhu, S. \& Moan, T. 2013. New insight into the wave-induced nonlinear vertical load effects of ultra-large container ships based on experiments. Journal of Marine Science and Technology 18: 87-114.

Zhu, S. \& Moan, T. 2014. Nonlinear effects from wave-induced maximum vertical bending moment on a flexible ultralarge containership model in severe head and oblique seas. Marine Structures 35: 1-25.

Zhu, T. \& Shigemi, T. 2003. Practical estimation methods of the design loads for primary structural members of bulk carriers. Marine Structures 16: 489-515.

Zhu, T. \& Shigemi, T. 2007. Design loads used for direct strength assessment of merchant ship structures. Transactions of the ASME 129: 120-130.

Zou, M.S., Wu, Y.S., Wu, W.W., Ye, Y.L. \& Tian, C. 2012. The three-dimensional hydroelasticity theory of ship structures in acoustic fluid of shallow sea. In Proc. $6^{\text {th }}$ Int. Conf. on Hydroelasticity in Marine Technology, Tokyo, Japan. 